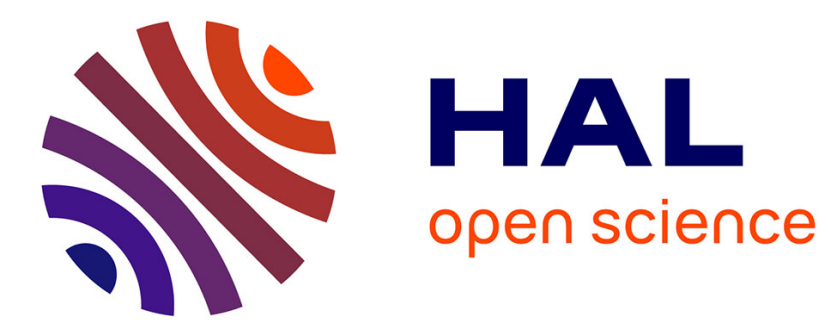

\title{
Long wave asymptotics for the Euler-Korteweg system
}

David Chiron, Sylvie Benzoni-Gavage

\section{To cite this version:}

David Chiron, Sylvie Benzoni-Gavage. Long wave asymptotics for the Euler-Korteweg system. Revista Matemática Iberoamericana, 2018, 34 (1), pp.245-304. 10.4171/RMI/985 . hal-01221714

\section{HAL Id: hal-01221714 \\ https://hal.science/hal-01221714}

Submitted on 28 Oct 2015

HAL is a multi-disciplinary open access archive for the deposit and dissemination of scientific research documents, whether they are published or not. The documents may come from teaching and research institutions in France or abroad, or from public or private research centers.
L'archive ouverte pluridisciplinaire HAL, est destinée au dépôt et à la diffusion de documents scientifiques de niveau recherche, publiés ou non, émanant des établissements d'enseignement et de recherche français ou étrangers, des laboratoires publics ou privés. 


\title{
Long wave asymptotics for the Euler-Korteweg system
}

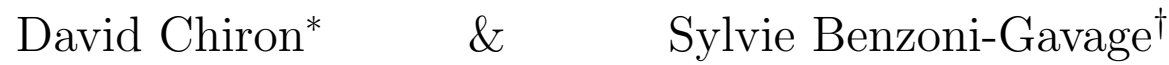

\begin{abstract}
The Euler-Korteweg system (EK) is a fairly general nonlinear waves model in mathematical physics that includes in particular the fluid formulation of the NonLinear Schrödinger equation (NLS). Several asymptotic regimes can be considered, regarding the length and the amplitude of waves. The first one is the free wave regime, which yields long acoustic waves of small amplitude. The other regimes describe a single wave or two counter propagating waves emerging from the wave regime. It is shown that in one space dimension those waves are governed either by inviscid Burgers or by Korteweg-de Vries equations, depending on the spatio-temporal and amplitude scalings. In higher dimensions, those waves are found to solve Kadomtsev-Petviashvili equations. Error bounds are provided in all cases. These results extend earlier work on defocussing (NLS) (and more specifically the Gross-Pitaevskii equation), and sheds light on the qualitative behavior of solutions to (EK), which is a highly nonlinear system of PDEs that is much less understood in general than (NLS).
\end{abstract}

Key-words: Euler-Korteweg system, capillary fluids, Korteweg de Vries equation, KadomtsevPetviashvili equation, weakly transverse Boussinesq system.

MSC (2010): 35Q35, 35Q53.

\section{Contents}

\begin{tabular}{llr}
\hline 1 & Introduction & 2
\end{tabular}

2 Preliminary material $\quad 5$

2.1 Statement of uniforms bounds . . . . . . . . . . . . . . . . . . . 5

2.2 Basic tools for the proof of uniform bounds . . . . . . . . . . . . . . . . . 7

2.3 Proof of uniform bounds in the potential case . . . . . . . . . . . . . . . . 11

2.4 Proof of uniform bounds in the general case . . . . . . . . . . . . . . . . . 17

${ }^{*}$ Laboratoire J.A. Dieudonné, CNRS UMR 7351, Université de Nice-Sophia Antipolis, Parc Valrose, 06108 Nice Cedex 02, France. (e-mail: chiron@unice.fr.)

†'Université de Lyon, Université Claude Bernard Lyon 1, CNRS UMR 5208, Institut Camille Jordan, 43 blvd du 11 novembre 1918, F-69622 Villeurbanne-Cedex, France. (e-mail: benzoni@math.univ-lyon1.fr.) 


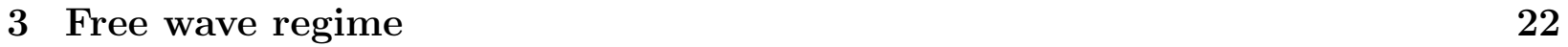

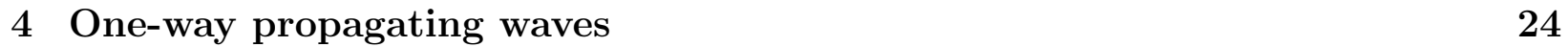

$4.1 \quad$ Statement of errors bounds in various asymptotic regimes . . . . . . . . . . . 24

4.2 Proof of error bounds for right-going waves . . . . . . . . . . . . . . . . . . . 26

4.3 The KdV regime for travelling waves . . . . . . . . . . . . . . . . . . . . . . . 35

5 Approximation by counter propagating waves 38

$5.1 \quad$ Statement of error bounds for counter propagating waves . . . . . . . . . . . 38

$5.2 \quad$ Detailed proof of error bounds for counter propagating waves . . . . . . . . . 39

$6 \quad$ The KP-I asymptotic regime 47

6.1 Main results . . . . . . . . . . . . . . . . . . . . . 47

6.2 Uniform bounds in the weakly transverse scaling . . . . . . . . . . . . . . . 50

6.3 Proof of Theorem 9 (ii) $\ldots \ldots \ldots \ldots$. . . . . . . . . . . . . . . . . . . . . . . . . . . . . . 52

6.4 Proof of Theorem 10 . . . . . . . . . . . . . . . . . . . . 57

\section{Introduction}

The Euler-Korteweg system is a dispersive perturbation of the Euler equations for compressible fluids. In its most general form, it reads

$(\mathrm{gEK})$

$$
\left\{\begin{array}{l}
\partial_{t} \rho+\nabla \cdot(\rho \mathbf{u})=0 \\
\partial_{t} \mathbf{u}+(\mathbf{u} \cdot \nabla) \mathbf{u}+\nabla(\delta \mathscr{F}[\rho])=0,
\end{array}\right.
$$

for a compressible fluid whose velocity field is $\mathbf{u}$, whose energy density $\mathscr{F}$ is allowed to depend on the fluid density $\rho$ and on its spatial gradient $\nabla \rho$, and $\delta \mathscr{F}[\rho]$ denotes the variational derivative of $\mathscr{F}$ at $\rho$. The standard Euler equations correspond to $\mathscr{F}=F(\rho)$ only, so that $\delta \mathscr{F}[\rho]=F^{\prime}(\rho)$ (and the pressure of the fluid is $p(\rho)=\rho F^{\prime}(\rho)-F(\rho)$ ). We are most interested in the classical form of the Euler-Korteweg system, which corresponds to

$$
\mathscr{F}=F(\rho)+\frac{1}{2} K(\rho)|\nabla \rho|^{2}
$$

where the so-called capillarity coefficient $K=K(\rho)$ is allowed to depend on the density $\rho$ in an arbitrary way, provided that $K$ is smooth and takes only positive values. In this case, (gEK) 'reduces' to

$$
\left\{\begin{array}{l}
\partial_{t} \rho+\nabla \cdot(\rho \mathbf{u})=0 \\
\partial_{t} \mathbf{u}+(\mathbf{u} \cdot \nabla) \mathbf{u}+\nabla(g(\rho))=\nabla\left(K(\rho) \Delta \rho+\frac{1}{2} K^{\prime}(\rho)|\nabla \rho|^{2}\right),
\end{array}\right.
$$

where $g \stackrel{\text { def }}{=} F^{\prime}$. Would $K$ be zero, the system (EK) would of course reduce to the standard Euler equations again, in which the sound speed is given by $\sqrt{\rho g^{\prime}(\rho)}$ as long as $g$ is a 
nondecreasing function of $\rho$. In the special case when $K(\rho)=1 /(4 \rho)$, the system (EK) can be derived from the (generalized) NonLinear Schrödinger equation (NLS) via the Madelung transform. An even more special case is $g(\varrho)=\varrho-1$, which corresponds to the GrossPitaevskii equation. In fact, (EK) is a ubiquitous system in mathematical physics, with various choices of $K$ and $g$, see for instance [3] for more details.

Associated with $(\mathrm{gEK})$ is a local conservation law for the total energy $\frac{1}{2} \rho|\mathbf{u}|^{2}+\mathscr{F}(\rho, \nabla \rho)$. However, the Cauchy problem for (gEK) has never been addressed for general energy densities $\mathscr{F}$. Because of analytical difficulties inherent in all systems involving high order derivatives (namely here, third order derivatives), the Cauchy problem analysis has been concentrated on (EK). The local well-posedness of (EK) is shown in [5] (one space dimension) and [4] (arbitrary space dimension). Our purpose here is to investigate the behavior of solutions of (EK) on longer times, by considering small perturbations of constant, thermodynamically stable states. By small we mean small amplitude perturbations that are significant on large space-time scales. By thermodynamically stable we mean reference densities $\varrho$ such that $g^{\prime}(\varrho)$ is positive. For any $\varrho$, the condition $g^{\prime}(\varrho)>0$ is equivalent to the hyperbolicity of the Euler equations at $(\varrho, 0)$ (or $(\varrho, \mathbf{u})$ for any velocity $\mathbf{u}$, by Galilean invariance) - and when applied to the fluid formulation of (NLS), it corresponds to what is known as the defocussing case. This paper aims at justifying several asymptotic limits regarding small amplitude, long wave solutions to the Euler-Korteweg system (EK), thus extending a series of recent work on (NLS) - and similar results known for the water wave equations.

The starting point is as follows. Constant states $(\varrho, 0)$ are obviously global solutions to $(\mathrm{EK})$ - and even $(\mathrm{gEK})$. Small amplitude perturbations $(\hat{\rho}, \hat{\mathbf{u}}))$ of $(\varrho, 0)$ are formally governed, at leading order, by the acoustic equations

$$
\left\{\begin{array}{l}
\partial_{t} \hat{\rho}+\varrho \nabla \cdot \hat{\mathbf{u}}=0 \\
\partial_{t} \hat{\mathbf{u}}+g^{\prime}(\varrho) \nabla \hat{\rho}=0 .
\end{array}\right.
$$

For $(\mathrm{gEK})$, it suffices to replace $g^{\prime}(\varrho)$ by $\frac{\partial^{2} \mathscr{F}}{\partial \rho^{2}}(\varrho, 0)$. We are only interested here in the case when these equations are well-posed, which amounts to requiring that $g^{\prime}(\varrho)>0$. From now on, we assume that $g$ is as smooth as necessary near $\varrho \neq 0$ - vacuum being excluded from our analysis -, that $g^{\prime}(\varrho)>0$, and we denote by

$$
\mathfrak{c} \stackrel{\text { def }}{=} \sqrt{\varrho g^{\prime}(\varrho)}>0
$$

the sound speed at $\varrho$. The acoustic equations admit particular solutions that are planar traveling waves $(\hat{\rho}, \hat{\mathbf{u}})=(\hat{\rho}, \hat{\mathbf{u}})(x-\mathbf{c} \mathbf{n} t)$ propagating with speed $\mathfrak{c}$ in any direction $\mathbf{n}$. A natural idea is to seek genuine solutions to $(\mathrm{EK})$ that are of small amplitude about $(\varrho, 0)$ and vary slowly in the frame attached to this linear wave.

In one space dimension, a prominent asymptotic regime corresponding to a weakly nonlinear limit can easily be identified by rescaling the solutions to the one D version of (EK) or even $(\mathrm{gEK})$ - as

$$
\rho(t, x)=\varrho+\varepsilon^{2} \tilde{\rho}(\theta, Y), \quad u(t, x)=\varepsilon^{2} \tilde{u}(\theta, Y), \quad \theta=\varepsilon^{3} t, \quad Y=\varepsilon(x-\mathfrak{c} t),
$$


for a small parameter $\varepsilon>0$ (here above, the scalar, fluid velocities are denoted by $u$ instead of the bold letter $\mathbf{u})$. Using that $\partial_{t}=\varepsilon^{3} \partial_{\theta}-\varepsilon \mathfrak{c} \partial_{Y}$ and $\partial_{x}=\varepsilon \partial_{Y}$, we see that for $(\rho, u)$ to solve $(\mathrm{gEK})$ in one $\mathrm{D}$ we must have

$$
\left\{\begin{array}{l}
\partial_{\theta} \tilde{\rho}-\frac{\mathfrak{c}}{\varepsilon^{2}} \partial_{Y} \tilde{\rho}+\frac{1}{\varepsilon^{2}} \partial_{Y}\left(\left(\varrho+\varepsilon^{2} \tilde{\rho}\right) \tilde{u}\right)=0 \\
\partial_{\theta} \tilde{u}-\frac{\mathfrak{c}}{\varepsilon^{2}} \partial_{Y} \tilde{u}+\tilde{u} \partial_{Y} \tilde{u}+\frac{1}{\varepsilon^{4}} \partial_{Y}\left(\delta \mathscr{F}\left[\varrho+\varepsilon^{2} \tilde{\rho}\right]\right)=0 .
\end{array}\right.
$$

Furthermore, by Taylor expansion we have

$$
\delta \mathscr{F}\left[\varrho+\varepsilon^{2} \tilde{\rho}\right]=\frac{\partial \mathscr{F}}{\partial \rho}(\varrho, 0)+\varepsilon^{2} \frac{\partial^{2} \mathscr{F}}{\partial \rho^{2}}(\varrho, 0) \tilde{\rho}+\frac{1}{2} \varepsilon^{4} \frac{\partial^{3} \mathscr{F}}{\partial \rho^{3}}(\varrho, 0) \tilde{\rho}^{2}-\varepsilon^{4} \frac{\partial^{2} \mathscr{F}}{\partial \rho_{x}^{2}}(\varrho, 0) \partial_{Y}^{2} \tilde{\rho}+\mathcal{O}\left(\varepsilon^{5}\right),
$$

which enables us to rewrite the system above as

$$
\left\{\begin{array}{l}
\partial_{\theta} \tilde{\rho}-\frac{\mathfrak{c}}{\varepsilon^{2}} \partial_{Y} \tilde{\rho}+\frac{\varrho}{\varepsilon^{2}} \partial_{Y} \tilde{u}+\partial_{Y}(\tilde{\rho} \tilde{u})=0 \\
\partial_{\theta} \tilde{u}-\frac{\mathfrak{c}}{\varepsilon^{2}} \partial_{Y} \tilde{u}+\tilde{u} \partial_{Y} \tilde{u}+\frac{\mathfrak{c}^{2}}{\varepsilon^{2} \varrho} \partial_{Y} \tilde{\rho}+\underline{\delta} \tilde{\rho} \partial_{Y} \tilde{\rho}-\underline{K} \partial_{Y}^{3} \tilde{\rho}=\mathcal{O}(\varepsilon)
\end{array}\right.
$$

with

$$
\mathfrak{c}^{2}=\varrho \frac{\partial^{2} \mathscr{F}}{\partial \rho^{2}}(\varrho, 0), \quad \underline{\delta} \stackrel{\text { def }}{=} \frac{\partial^{3} \mathscr{F}}{\partial \rho^{3}}(\varrho, 0), \quad \underline{K} \stackrel{\text { def }}{=} \frac{\partial^{2} \mathscr{F}}{\partial \rho_{x}^{2}}(\varrho, 0) .
$$

If we go on at a formal level, we find by inspecting the $\mathcal{O}\left(\varepsilon^{-2}\right)$ terms that necessarily $\mathfrak{c} \tilde{\rho} \approx \varrho \tilde{u}$, and by taking a linear combination of the $\mathcal{O}(1)$ terms in the system above, we see that $\mathrm{w} \stackrel{\text { def }}{=} \frac{1}{2}\left(\tilde{\rho}+\frac{\varrho}{\mathfrak{c}} \tilde{u}\right)$ should approximately satisfy the Korteweg-de Vries (KdV) equation

$$
\partial_{\theta} \mathrm{w}+\Gamma \mathrm{w} \partial_{Y} \mathrm{w}=\kappa \partial_{Y}^{3} \mathrm{w}
$$

with

$$
\Gamma \stackrel{\text { def }}{=} \frac{3 \mathfrak{c}}{2 \varrho}+\frac{\varrho \underline{\delta}}{2 \mathfrak{c}}, \quad \kappa \stackrel{\text { def }}{=} \frac{\varrho \underline{K}}{2 \mathfrak{c}} .
$$

When dealing with $(\mathrm{EK})$, we merely have $\underline{\delta}=g^{\prime \prime}(\varrho)$ and $\underline{K}=K(\varrho)$. Of course, if $\underline{K}=0$ we recover the well-known Burgers equation

$$
\partial_{\theta} \mathrm{w}+\Gamma \mathrm{w} \partial_{Y} \mathrm{w}=0
$$

as an asymptotic equation for the weakly nonlinear wave solutions to the Euler equations. The parameter $\Gamma$ is nonzero provided that the characteristic fields are genuinely nonlinear in the neighborhood of $\varrho$. Indeed, both characteristic fields of the Euler equations are genuinely nonlinear in the neighborhood of $\varrho$ if and only if $\partial_{\rho}\left(\rho \sqrt{\rho g^{\prime}(\rho)}\right)_{\mid \varrho} \neq 0$, and by definition of $\mathfrak{c}$ we have

$$
\partial_{\rho}\left(\rho \sqrt{\rho g^{\prime}(\rho)}\right)_{\mid \varrho}=\mathfrak{c}+\frac{\varrho}{2 \mathfrak{c}}\left(\frac{\mathfrak{c}^{2}}{\varrho}+\varrho g^{\prime \prime}(\varrho)\right)=\varrho \Gamma \text {. }
$$


In fact, the dimensionless number $\varrho \Gamma / \mathfrak{c}$ measures nonlinearity of pressure waves, and is positive in standard fluids.

More generally, in order to find relevant asymptotic regimes, we seek solutions to (EK) of the form

$$
\rho(t, x)=\varrho+\eta \hat{\rho}(\varepsilon t, \varepsilon x), \quad \mathbf{u}(t, x)=\eta \hat{\mathbf{u}}(\varepsilon t, \varepsilon x),
$$

with $\eta>0$ and $\varepsilon>0$ some small, a priori independent parameters. The former gives an order of magnitude for the amplitude of solutions, and $1 / \varepsilon$ is a spatio-temporal scale on which solutions are supposed to vary significantly. The distinction between the time evolution scale and the spatial scale comes later in the analysis.

After the linear wave regime considered in Section 3, the Korteweg-de Vries and the Burgers regimes described above - which correspond to the special case $\eta=\varepsilon$ and $\eta=\varepsilon^{2}$ in (2) - are fully justified in Section 4 for solutions to (EK) with well-prepared initial data, along with alternate regimes in which dispersive effects are weaker - i.e when $\varepsilon^{2} \ll \eta$. Section 5 is devoted to more general initial data, and asymptotic regimes obtained by decoupling left-going and right-going waves. Finally, multidimensional, weakly transverse effects are taken into account in Section 6, in which we justify the so-called Kadomtsev-Petviashvili regime for $(\mathrm{EK})$.

\section{Preliminary material}

\section{$2.1 \quad$ Statement of uniforms bounds}

The ansatz (2) obviously transforms (EK) into the rescaled system

$$
\left(\mathrm{EK}_{\varepsilon, \eta}\right) \quad\left\{\begin{aligned}
\partial_{T} \hat{\rho}+ & \nabla_{X} \cdot((\varrho+\eta \hat{\rho}) \hat{\mathbf{u}})=0 \\
\partial_{T} \hat{\mathbf{u}}+ & \eta\left(\hat{\mathbf{u}} \cdot \nabla_{X}\right) \hat{\mathbf{u}}+g^{\prime}(\varrho+\eta \hat{\rho}) \nabla_{X} \hat{\rho} \\
& =\varepsilon^{2} \nabla_{X}\left(K(\varrho+\eta \hat{\rho}) \Delta_{X} \hat{\rho}+\frac{\eta}{2} K^{\prime}(\varrho+\eta \hat{\rho})\left|\nabla_{X} \hat{\rho}\right|^{2}\right)
\end{aligned}\right.
$$

where $T=\varepsilon t, X=\varepsilon x$. Note that the acoustic equations are formally obtained by setting $\eta=0, \varepsilon=0$ in $\left(\mathrm{EK}_{\varepsilon, \eta}\right)$. For $\eta>0, \varepsilon>0$, the local well-posedness of the Cauchy problem associated with $\left(\mathrm{EK}_{\varepsilon, \eta}\right)$ follows from the following result.

Theorem 1 ([4]) Let us take $s>1+\frac{d}{2}$, and $\left(\rho^{\mathrm{in}}, \mathbf{u}^{\mathrm{in}}\right) \in(\varrho, 0)+H^{s+1}\left(\mathbb{R}^{d}\right) \times\left(H^{s}\left(\mathbb{R}^{d}\right)\right)^{d}$ such that $\rho^{\text {in }}$ is positive and bounded by below in $\mathbb{R}^{d}$. Then, there exists a time $t_{*}>0$ such that the system (EK) possesses a unique solution

$$
(\rho, \mathbf{u}) \in(\varrho, 0)+\mathscr{C}\left(\left[0, t_{*}\right], H^{s+1}\left(\mathbb{R}^{d}\right) \times\left(H^{s}\left(\mathbb{R}^{d}\right)\right)^{d}\right) \cap \mathscr{C}^{1}\left(\left[0, t_{*}\right], H^{s-1}\left(\mathbb{R}^{d}\right) \times\left(H^{s-2}\left(\mathbb{R}^{d}\right)\right)^{d}\right)
$$

such that $(\rho, \mathbf{u})(0)=\left(\rho^{\mathrm{in}}, \mathbf{u}^{\mathrm{in}}\right)$. Moreover, the mapping $\left(\rho^{\mathrm{in}}, \mathbf{u}^{\mathrm{in}}\right) \mapsto(\rho, \mathbf{u})$ is continuous. 
However, we need refined estimates of solutions that: 1) keep track of the parameters $(\eta, \varepsilon) ; 2)$ take into account the nonlinear term $g^{\prime}(\varrho+\eta \hat{\rho}) \nabla_{X} \hat{\rho}$ - and not as a source term as in [4] -, which will be possible thanks to the positivity of $g^{\prime}(\varrho)$. Furthermore, the following result implies that, as expected, the smaller the initial data, the longer the time of existence of the solution.

Theorem 2 Let $s$ be a real number greater than $1+\frac{d}{2}$ and $\eta \in(0,1]$. For $\varepsilon>0$ and $M>0$, we consider

$$
\mathcal{B}_{s, \varepsilon}(M) \stackrel{\text { def }}{=}\left\{(\hat{\rho}, \hat{\mathbf{u}}) \in H^{s+1}\left(\mathbb{R}^{d}\right) \times\left(H^{s}\left(\mathbb{R}^{d}\right)\right)^{d} ;\|(\hat{\rho}, \hat{\mathbf{u}})\|_{\left(H^{s}\left(\mathbb{R}^{d}\right)\right)^{d+1}}+\varepsilon\|\hat{\rho}\|_{H^{s+1}\left(\mathbb{R}^{d}\right)} \leqslant M\right\} .
$$

If $\varrho>0, g^{\prime}(\varrho)>0$, and $\left(\hat{\rho}^{\mathrm{in}}, \hat{\mathbf{u}}^{\mathrm{in}}\right) \in \mathcal{B}_{s, \varepsilon}(M)$, then there exists $T_{*}>0$, depending only on $M$, $s$ and $d$, such that the maximal solution to $\left(\mathrm{EK}_{\varepsilon, \eta}\right)$ in $H^{s+1}\left(\mathbb{R}^{d}\right) \times\left(H^{s}\left(\mathbb{R}^{d}\right)\right)^{d}$ with $(\hat{\rho}, \hat{\mathbf{u}})(0)=$ $\left(\hat{\rho}^{\text {in }}, \hat{\mathbf{u}}^{\text {in }}\right)$ exists at least on $\left[0, T_{*} / \eta\right]$, and $(\hat{\rho}, \hat{\mathbf{u}})(T) \in \mathcal{B}_{s, \varepsilon}(2 M)$ for all $T \in\left[0, T_{*} / \eta\right]$.

A similar result is shown in [6, Theorem 1] for the hydrodynamical formulation of the Gross-Pitaevskii equation obtained with the Madelung transform. However, it is stated in terms of $\|(\hat{\rho}, \hat{\mathbf{u}})\|_{H^{s+1} \times H^{s}}$ instead of $\|(\hat{\rho}, \hat{\mathbf{u}})\|_{H^{s}}+\varepsilon\|\hat{\rho}\|_{H^{s+1}}$ (with our notations), which seems to be a slight mistake. A priori estimates rely indeed on Proposition 1 in [6], in which some quantity denoted by $z$ is controlled in $H^{s}$, but the imaginary part of $z$ is $2 \frac{\nabla \rho}{\rho}$ with $\rho=1+\varepsilon \hat{\rho}$, so that only $\varepsilon\|\hat{\rho}\|_{H^{s+1}}$ is controlled. The estimate in [6. Theorem 2] should certainly be modified accordingly. Apart from this harmless correction, the main novelty here compared to [6] is twofold. First, the capillarity is arbitrary, which means in particular that it is not assumed to be proportional to $1 / \rho$. As already known from [4], the a priori estimates are much trickier when $\rho K(\rho)$ is not constant. The other point is that we do not assume the vector field $\mathbf{u}$ to be potential - unlike what happens when dealing with the fluid formulation of (NLS). This is again known to make a priori estimates more complicated. However, it is important to deal with general capillarities and velocity fields for various applications.

Remark 1 The special case $\eta=\varepsilon^{2}$ is called the Boussinesq regime. If, in addition, the capillarity $K$ is a positive constant and $g$ is a convex, quadratic polynomial (i.e. $g^{\prime}=$ constant $>0)$, then $\left(\mathrm{EK}_{\varepsilon, \varepsilon^{2}}\right)$ belongs to the $(a, b, c, d)$-class of Boussinesq type systems as introduced in [9] and [10], with $a=b=d=0$ and $c=-K<0$. In this case, the existence and uniqueness of (strong) solutions on the time scale $\varepsilon^{-2}$ has been shown in [24], using hyperbolic techniques (see Theorem 1.1 in [24], case (12) in the sense of their definition 1.2). Our own result (Theorem 2 here above) applied to $\eta=\varepsilon^{2}, K=$ constant $>0, g^{\prime}=$ constant $>0$, provides an alternate proof of theirs in that case.

Theorem 2 is a building block for the rigorous justification of asymptotic regimes. We need some material in order to prove it. 


\subsection{Basic tools for the proof of uniform bounds}

As in [4], we shall derive uniform Sobolev bounds through an extended formulation of the system (EK). The idea is to introduce the complex-valued unknown $\mathbf{z}=\mathbf{u}+i \mathbf{w}$ that is naturally involved in the global energy

$$
\mathscr{E}=\int_{\mathbb{R}^{d}}\left(\frac{1}{2} \rho|\mathbf{u}|^{2}+F(\rho)+\frac{1}{2} K(\rho)|\nabla \rho|^{2}\right) d x .
$$

This integral is indeed well defined provided that we redefine $F(\rho) \stackrel{\text { def }}{=} \int_{\varrho}^{\rho} g$, which merely amounts to adding a constant to $F$ so that $F(\varrho)=0$, and conserved along (smooth) solutions $(\rho, \mathbf{u})$ to $(\mathrm{EK})$ that tend to $(\varrho, 0)$ sufficiently fast at infinity. In addition, we can write

$$
\frac{1}{2} \rho|\mathbf{u}|^{2}+\frac{1}{2} K(\rho)|\nabla \rho|^{2}=\frac{1}{2} \rho|\mathbf{z}|^{2} \quad \text { with } \mathbf{z}=\mathbf{u}+i \mathbf{w}, \mathbf{w} \stackrel{\text { def }}{=} \sqrt{\frac{K(\rho)}{\rho}} \nabla \rho .
$$

Then, if we introduce

$$
a(\rho) \stackrel{\text { def }}{=} \sqrt{\rho K(\rho)}, \quad b(\rho) \stackrel{\text { def }}{=} \frac{\rho g^{\prime}(\rho)}{a(\rho)},
$$

by differentiating the first equation in $(\mathrm{EK})$ we obtain the following, equivalent system for $(\rho, \mathbf{z})$,

$$
\left\{\begin{array}{l}
\partial_{t} \rho+\nabla \cdot(\rho \mathbf{u})=0 \\
\partial_{t} \mathbf{z}+(\mathbf{u} \cdot \nabla) \mathbf{z}+i(\nabla \mathbf{z}) \mathbf{w}+b(\rho) \mathbf{w}+i \nabla(a(\rho) \nabla \cdot \mathbf{z})=0
\end{array}\right.
$$

in which the notation $(\nabla \mathbf{z}) \mathbf{w}$ stands for the standard product of the matrix-valued function $\nabla \mathbf{z}=\left(\partial_{j} \mathbf{z}_{k}\right)_{1 \leqslant j, k \leqslant d}$ and the vector field $\mathbf{w}$, so that

$$
((\nabla \mathbf{z}) \mathbf{w})_{j}=\sum_{k=1}^{d}\left(\partial_{j} \mathbf{z}_{k}\right) \mathbf{w}_{k}=\left(\partial_{j} \mathbf{z}\right) \cdot \mathbf{w}
$$

where we use the notation $\mathbf{u} \cdot \mathbf{v}=\sum_{j=1}^{d} u_{j} v_{j}$ for $\mathbf{u}, \mathbf{v} \in \mathbb{C}^{d}$. The scaling in (2) urges us to define

$$
\hat{\mathbf{w}} \stackrel{\text { def }}{=} \varepsilon \sqrt{\frac{K(\rho)}{\rho}} \nabla_{X} \hat{\rho}, \quad \hat{\mathbf{z}} \stackrel{\text { def }}{=} \hat{\mathbf{u}}+i \hat{\mathbf{w}}
$$

so that $\mathbf{z}(t, x)=\eta(\hat{\mathbf{u}}+i \hat{\mathbf{w}})(T, X)=\eta \hat{\mathbf{z}}(T, X)$, and (ES) equivalently reads

$$
\left(\mathrm{ES}_{\varepsilon, \eta}\right) \quad\left\{\begin{array}{l}
\partial_{T} \rho+\eta \nabla_{X} \cdot(\rho \hat{\mathbf{u}})=0 \\
\partial_{T} \hat{\mathbf{z}}+\eta\left(\hat{\mathbf{u}} \cdot \nabla_{X}\right) \hat{\mathbf{z}}+i \eta\left(\nabla_{X} \hat{\mathbf{z}}\right) \hat{\mathbf{w}}+\frac{1}{\varepsilon} b(\rho) \hat{\mathbf{w}}+i \varepsilon \nabla_{X}\left(a(\rho) \nabla_{X} \cdot \hat{\mathbf{z}}\right)=0 .
\end{array}\right.
$$


Our main purpose here is to derive some a priori estimates for solutions to $\left(\mathrm{ES}_{\varepsilon, \eta}\right)$ that are valid uniformly in $(\varepsilon, \eta)$. In this respect, we are going to use a modified version of the energy

$$
\mathscr{E}=\frac{\eta^{2}}{2 \varepsilon^{d}} \int_{\mathbb{R}^{d}}\left(\rho|\hat{\mathbf{z}}|^{2}+\frac{2}{\eta^{2}} F(\varrho+\eta \hat{\rho})\right) d X .
$$

Recalling that we have redefined $F$ so that $F(\varrho)=0$, omitting the linear term in $\hat{\rho}$ in the Taylor expansion of $F$ about $\varrho$, which is justified by the fact this term does not contribute - at least to the lowest order - to a priori estimates since $\hat{\rho}$ is conserved, and removing the factor $\eta^{2} \varepsilon^{-d}$, we arrive at the modified energy

$$
E_{0}(\hat{\rho}, \hat{\mathbf{z}}) \stackrel{\text { def }}{=} \frac{1}{2} \int_{\mathbb{R}^{d}} \rho|\hat{\mathbf{z}}|^{2}+g^{\prime}(\rho) \hat{\rho}^{2} d X, \quad \rho=\varrho+\eta \hat{\rho} .
$$

Clearly, even though $E_{0}$ depends on $\eta$ through $\rho$, the assumption $g^{\prime}(\varrho)>0$ ensures that $\sqrt{E_{0}[\hat{\rho}, \hat{\mathbf{z}}]}$ is equivalent to the $L^{2}$ norm of $(\hat{\rho}, \hat{\mathbf{z}})$ as long as $\rho$ and $g^{\prime}(\rho)$ remain bounded and bounded away from zero. Moreover, going back to (3), we may see $E_{0}$ as a functional applied to $(\hat{\rho}, \hat{\mathbf{u}})$, and, as such, $E_{0}[\hat{\rho}, \hat{\mathbf{u}}]$ enjoys the following estimates.

Proposition 1 Let $r \in(0, \varrho / 2]$ be such that $g^{\prime}(\rho)>0$ and $K(\rho)>0$ if $|\rho-\varrho| \leqslant r$. Then for all $(\hat{\rho}, \hat{\mathbf{u}}) \in H^{1} \times L^{2}$ such that $\|\hat{\rho}\|_{L^{\infty}} \leqslant r$, for all $\eta \in(0,1]$, for all $\varepsilon>0$,

$$
c_{0}\left(\|\hat{\mathbf{u}}\|_{L^{2}}^{2}+\|\hat{\rho}\|_{L^{2}}^{2}+\varepsilon^{2}\|\hat{\rho}\|_{H^{1}}^{2}\right) \leqslant E_{0}[\hat{\rho}, \hat{\mathbf{u}}] \leqslant C_{0}\left(\|\hat{\mathbf{u}}\|_{L^{2}}^{2}+\|\hat{\rho}\|_{L^{2}}^{2}+\varepsilon^{2}\|\hat{\rho}\|_{H^{1}}^{2}\right),
$$

where $c_{0}>0$ and $C_{0}>0$ depend only on $r$ (and the functions $g, K$ ).

Proof. We have these inequalities with, explicitly,

$c_{0} \stackrel{\text { def }}{=} \frac{1}{2} \min _{|\rho-\varrho| \leqslant r} \min \left(\rho, g^{\prime}(\rho), \sqrt{K(\rho) / \rho)}\right), \quad C_{0} \stackrel{\text { def }}{=} \frac{1}{2} \max _{|\rho-\varrho| \leqslant r} \max \left(\rho, g^{\prime}(\rho), \sqrt{K(\rho) / \rho)}\right)$.

Now, the following, zero-th order a priori estimate is reminiscent of the fact that the exact energy $\mathscr{E}$ is conserved along solutions of (EK).

Proposition 2 Let $\eta \in(0,1]$. Assume that $(\hat{\rho}, \hat{\mathbf{u}}) \in \mathscr{C}\left(\left[0, t_{*}\right], H^{s+1}\left(\mathbb{R}^{d}\right) \times\left(H^{s}\left(\mathbb{R}^{d}\right)\right)^{d}\right) \cap$ $\mathscr{C}^{1}\left(\left[0, t_{*}\right], H^{s-1}\left(\mathbb{R}^{d}\right) \times\left(H^{s-2}\left(\mathbb{R}^{d}\right)\right)^{d}\right)$ is a solution of $\left(\mathrm{EK}_{\varepsilon, \eta}\right)$ for some $s>1+d / 2$, such that $\|\hat{\rho}\|_{L^{\infty}} \leqslant r$, where $r$ is as in Proposition 1 . Then there exists $C>0$ depending only on $r$ such that

$$
\frac{d}{d T} E_{0}[\hat{\rho}, \hat{\mathbf{u}}] \leqslant C \eta\left\|\left(\nabla_{X} \hat{\rho}, \nabla_{X} \mathbf{u}\right)\right\|_{L^{\infty}} E_{0}[\hat{\rho}, \hat{\mathbf{u}}]
$$

Proof. Of course, we are going to use that $(\hat{\rho}, \hat{\mathbf{z}}=\hat{\mathbf{u}}+i \hat{\mathbf{w}})$ solves $\left(\mathrm{ES}_{\varepsilon, \eta}\right)$ if $\rho \hat{\mathbf{w}}=\varepsilon a(\rho) \nabla_{X} \hat{\rho}$ - this equality just being a different way of writing (3). Recall also that $a(\rho) b(\rho)=\rho g^{\prime}(\rho)$. The notation $\langle\cdot, \cdot\rangle$ will stand everywhere for real-valued inner products, and more precisely 
$\langle\mathbf{z}, \boldsymbol{\zeta}\rangle=\frac{1}{2} \sum_{j=1}^{d}\left(\overline{\mathbf{z}}_{j} \boldsymbol{\zeta}_{j}+\mathbf{z}_{j} \overline{\boldsymbol{\zeta}}_{j}\right)$ for all $\mathbf{z}, \boldsymbol{\zeta} \in \mathbb{C}^{d}$ (whatever $d$, including $d=1$ ). Using $\left(\operatorname{ES}_{\varepsilon, \eta}\right)$, we find by straightforward differentiation that

$$
\begin{aligned}
2 \frac{d}{d T} E_{0}[\hat{\rho}, \hat{\mathbf{u}}]= & -\eta \int \nabla_{X} \cdot(\rho \hat{\mathbf{u}})|\hat{\mathbf{z}}|^{2}-\eta \int \rho \hat{\mathbf{u}} \cdot \nabla_{X}|\hat{\mathbf{z}}|^{2}-2 \eta \int \rho\left\langle i\left(\nabla_{X} \hat{\mathbf{z}}\right) \hat{\mathbf{w}}, \hat{\mathbf{z}}\right\rangle \\
& -\frac{2}{\varepsilon} \int \rho b(\rho)\langle\hat{\mathbf{w}}, \hat{\mathbf{u}}\rangle-2 \varepsilon \int \rho\left\langle i \nabla_{X}\left(a(\rho) \nabla_{X} \cdot \hat{\mathbf{z}}\right), \hat{\mathbf{z}}\right\rangle \\
& +\int \hat{\mathbf{u}} \cdot \nabla_{X}\left(2 \rho g^{\prime}(\rho) \hat{\rho}+\eta\left(\rho g^{\prime \prime}(\rho)-g^{\prime}(\rho)\right) \hat{\rho}^{2}\right) .
\end{aligned}
$$

By the relations recalled above and an integration by part, this reduces to

$$
\begin{aligned}
2 \frac{d}{d T} E_{0}[\hat{\rho}, \hat{\mathbf{u}}]= & -2 \varepsilon \int a(\rho)\left\langle i\left(\nabla_{X} \hat{\mathbf{z}}\right) \cdot \nabla_{X} \rho, \hat{\mathbf{z}}\right\rangle \\
& -2 \int \rho g^{\prime}(\rho)\left(\hat{\mathbf{u}} \cdot \nabla_{X} \hat{\rho}\right)+2 \varepsilon \int a(\rho)\left\langle i \nabla_{X} \cdot \hat{\mathbf{z}}, \hat{\mathbf{z}} \cdot \nabla_{X} \rho\right\rangle \\
& +\int \hat{\mathbf{u}} \cdot \nabla_{X}\left(2 \rho g^{\prime}(\rho) \hat{\rho}+\eta\left(\rho g^{\prime \prime}(\rho)-g^{\prime}(\rho)\right) \hat{\rho}^{2}\right) .
\end{aligned}
$$

Now, using that $a(\rho) \nabla \rho$ is potential, we see that the $\varepsilon$-terms cancel out, and simplifying/integrating by parts the remaining terms we obtain

$$
2 \frac{d}{d T} E_{0}[\hat{\rho}, \hat{\mathbf{u}}]=2 \eta \int \partial_{\rho}\left(\rho g^{\prime}(\rho)\right) \hat{\rho} \hat{\mathbf{u}} \cdot \nabla_{X} \hat{\rho}-\eta \int\left(\rho g^{\prime \prime}(\rho)-g^{\prime}(\rho)\right) \hat{\rho}^{2} \nabla_{X} \hat{\mathbf{u}} .
$$

The claimed inequality thus holds true with

$$
C=\frac{1}{c_{0}} \max _{|\rho-\varrho| \leqslant r}\left(\left|\partial_{\rho}\left(\rho g^{\prime}(\rho)\right)\right|+\left|\rho g^{\prime \prime}(\rho)-g^{\prime}(\rho)\right|\right) .
$$

Since it involves the $W^{1, \infty}$ norm of the solution $(\hat{\rho}, \hat{\mathbf{u}})$, the estimate in $(2)$ is clearly not sufficient to get a priori estimates without loss of derivatives. In order to close the estimates, we need higher order ones. If $s$ is a large enough integer, we may use

$$
\begin{gathered}
E_{s}[\hat{\rho}, \hat{\mathbf{z}}] \stackrel{\text { def }}{=} \sum_{\sigma=0}^{s} \dot{E}_{\sigma}[\hat{\rho}, \hat{\mathbf{z}}], \\
\dot{E}_{\sigma}[\hat{\rho}, \hat{\mathbf{z}}] \stackrel{\text { def }}{=} \sum_{\substack{\alpha \in \mathbb{N}_{0}^{d},|\alpha|=\sigma}} \frac{\sigma !}{\alpha !} \int_{\mathbb{R}^{d}} \frac{1}{2} a(\rho)^{\sigma}\left(\rho\left|\partial^{\alpha} \hat{\mathbf{z}}\right|^{2}+g^{\prime}(\rho)\left(\partial^{\alpha} \hat{\rho}\right)^{2}\right) d X, \quad \rho=\varrho+\eta \hat{\rho},
\end{gathered}
$$

where $\partial^{\alpha}$ stands for $\partial^{|\alpha|} / \partial X_{1}^{\alpha_{1}} \ldots \partial X_{d}^{\alpha_{d}}$. The coefficients $\frac{\sigma !}{\alpha !}$ here above, as well as the weights $a^{\sigma}$, are chosen so as to eliminate bad terms in our a priori estimates, as we shall see. The usefulness of these estimates will be based on the following, in which $E_{s}$ is viewed as a functional applied to $(\hat{\rho}, \hat{\mathbf{u}})$, by using (3) as for $E_{0}$. 
Proposition 3 Let $s$ be a positive integer. Let $r \in(0, \varrho / 2]$ be as in Proposition[1. Then for all $(\hat{\rho}, \hat{\mathbf{u}}) \in H^{s+1}\left(\mathbb{R}^{d}\right) \times\left(H^{s}\left(\mathbb{R}^{d}\right)\right)^{d}$ such that $\|\hat{\rho}\|_{W^{1, \infty}} \leqslant r$, for all $\eta \in(0,1]$, for all $\varepsilon>0$,

$$
c\left(\|\hat{\mathbf{u}}\|_{H^{s}}^{2}+\|\hat{\rho}\|_{H^{s}}^{2}+\varepsilon^{2}\|\hat{\rho}\|_{H^{s+1}}^{2}\right) \leqslant E_{s}[\hat{\rho}, \hat{\mathbf{u}}] \leqslant C\left(\|\hat{\mathbf{u}}\|_{H^{s}}^{2}+\|\hat{\rho}\|_{H^{s}}^{2}+\varepsilon^{2}\|\hat{\rho}\|_{H^{s+1}}^{2}\right),
$$

where $c>0$ and $C>0$ depend only on $r, s, d$ (and the functions $g, K$ ).

Proof. As in the proof of Proposition 1, we readily see that

$$
c_{\sigma}\left(\left\|\partial^{\alpha} \hat{\mathbf{z}}\right\|_{L^{2}}^{2}+\left\|\partial^{\alpha} \hat{\rho}\right\|_{L^{2}}^{2}\right) \leqslant \int_{\mathbb{R}^{d}} a(\rho)^{\sigma}\left(\rho\left|\partial^{\alpha} \hat{\mathbf{z}}\right|^{2}+g^{\prime}(\rho)\left(\partial^{\alpha} \hat{\rho}\right)^{2}\right) d X \leqslant C_{\sigma}\left(\left\|\partial^{\alpha} \hat{\mathbf{z}}\right\|_{L^{2}}^{2}+\left\|\partial^{\alpha} \hat{\rho}\right\|_{L^{2}}^{2}\right),
$$

with

$c_{\sigma} \stackrel{\text { def }}{=} \min _{|\rho-\varrho| \leqslant r}\left(a(\rho)^{\sigma} \min \left(\rho, g^{\prime}(\rho), \sqrt{K(\rho) / \rho)}\right)\right), C_{\sigma} \stackrel{\text { def }}{=} \max _{|\rho-\rho| \leqslant r}\left(a(\rho)^{\sigma} \max \left(\rho, g^{\prime}(\rho), \sqrt{K(\rho) / \rho)}\right)\right)$.

By summation we thus find $c_{s}>0$ and $C_{s}>0$ such that

$$
c_{s}\left(\|\hat{\mathbf{z}}\|_{H^{s}}^{2}+\|\hat{\rho}\|_{H^{s}}^{2}\right) \leqslant E_{s}[\hat{\rho}, \hat{\mathbf{u}}] \leqslant C_{s}\left(\|\hat{\mathbf{z}}\|_{H^{s}}^{2}+\|\hat{\rho}\|_{H^{s}}^{2}\right) .
$$

So the only point is to check that $\|\hat{\mathbf{z}}\|_{H^{s}}^{2}+\|\hat{\rho}\|_{H^{s}}^{2}$ is equivalent to $\|\hat{\mathbf{u}}\|_{H^{s}}^{2}+\|\hat{\rho}\|_{H^{s}}^{2}+\varepsilon^{2}\|\hat{\rho}\|_{H^{s+1}}^{2}$ when $\hat{\mathbf{z}}=\hat{\mathbf{u}}+i \hat{\mathbf{w}}, \hat{\mathbf{w}}=\varepsilon c(\varrho+\eta \hat{\rho}) \nabla_{X} \hat{\rho}$ for some smooth function $c$ - here $c(\rho)=\sqrt{K(\rho) / \rho}$. This comparison relies on rather standard estimates, which are stated in the appendix (Proposition A.1) for convenience. Indeed, we have

$$
\begin{gathered}
\left\|c(\varrho+\eta \hat{\rho}) \nabla_{X} \hat{\rho}\right\|_{H^{s}} \leqslant c(\varrho)\left\|\nabla_{X} \hat{\rho}\right\|_{H^{s}}+\gamma\left\|c^{\prime}\right\|_{W^{s, \infty}([\varrho-r, \varrho+r])}\left(1+\|\hat{\rho}\|_{L^{\infty}}\right)^{\sigma}\left\|\nabla_{X} \hat{\rho}\right\|_{L^{\infty}}\|\hat{\rho}\|_{H^{s}} \\
+2 \gamma\|c\|_{L^{\infty}([\varrho-r, \varrho+r])}\left\|\nabla_{X} \hat{\rho}\right\|_{H^{s}} \\
\leqslant C\left(\|\hat{\rho}\|_{\left.W^{1, \infty}\right)}\|\hat{\rho}\|_{H^{s+1}}\right.
\end{gathered}
$$

and in a similar way, using the notation $d$ for $1 / c$,

$$
\begin{gathered}
\varepsilon\left\|\nabla_{X} \hat{\rho}\right\|_{H^{s}} \leqslant d(\varrho)\|\hat{\mathbf{w}}\|_{H^{s}}+\gamma\left\|d^{\prime}\right\|_{W^{s, \infty}([\varrho-r, \varrho+r])}\left(1+\|\hat{\rho}\|_{L^{\infty}}\right)^{\sigma}\|\hat{\mathbf{w}}\|_{L^{\infty}}\|\hat{\rho}\|_{H^{s}} \\
+2 \gamma\|c\|_{L^{\infty}([\varrho-r, \varrho+r])}\|\hat{\mathbf{w}}\|_{H^{s}} \\
\leqslant C\left(\|(\hat{\rho}, \hat{\mathbf{w}})\|_{L^{\infty}}\right)\|\hat{\rho}\|_{H^{s}}+C(r)\|\hat{\mathbf{w}}\|_{H^{s}} .
\end{gathered}
$$

Here above, $\gamma$ stands for a 'universal' constant (depending only on $s$ and $d$ ), and $C(q)$ stands for a positive number depending only on $q$, whatever the quantity $q$. We can thus conclude that

$$
\begin{gathered}
\|\hat{\mathbf{z}}\|_{H^{s}}^{2}+\|\hat{\rho}\|_{H^{s}}^{2} \leqslant \max \left(1, C\left(\|\hat{\rho}\|_{W^{1, \infty}}\right)^{2}\right)\left(\|\hat{\mathbf{u}}\|_{H^{s}}^{2}+\|\hat{\rho}\|_{H^{s}}^{2}+\varepsilon^{2}\|\hat{\rho}\|_{H^{s+1}}^{2}\right), \\
\|\hat{\mathbf{u}}\|_{H^{s}}^{2}+\|\hat{\rho}\|_{H^{s}}^{2}+\varepsilon^{2}\|\hat{\rho}\|_{H^{s+1}}^{2} \leqslant \max \left(1+2 C\left(\|(\hat{\rho}, \hat{\mathbf{w}})\|_{L^{\infty}}\right)^{2}, C(r)^{2}\right)\left(\|\mathbf{z}\|_{H^{s}}^{2}+\|\hat{\rho}\|_{H^{s}}^{2}\right) .
\end{gathered}
$$




\subsection{Proof of uniform bounds in the potential case}

In this section, we are going to prove that for any integer $s>1+d / 2, E_{s}$ enjoys an a priori estimate that is similar to the one in Proposition 2 for $E_{0}$, at least when the velocity vector field $\mathbf{u}$ is potential. We start with this simpler case for the sake of clarity - all computations below are detailed enough to be readable without a pen. As was noticed in [4], the fact that $\mathbf{u}$ is potential or, equivalently, that $\mathbf{u}$ is curl-free is preserved along (smooth) solutions. So it is sufficient to assume that the initial velocity field is curl-free for these computations to be valid.

Proposition 4 Assume that $(\hat{\rho}, \hat{\mathbf{u}}) \in \mathscr{C}\left(\left[0, t_{*}\right], H^{s+1}\left(\mathbb{R}^{d}\right) \times\left(H^{s}\left(\mathbb{R}^{d}\right)\right)^{d}\right) \cap \mathscr{C}^{1}\left(\left[0, t_{*}\right], H^{s-1}\left(\mathbb{R}^{d}\right) \times\right.$ $\left.\left(H^{s-2}\left(\mathbb{R}^{d}\right)\right)^{d}\right)$ is a solution of $\left(\mathrm{EK}_{\varepsilon, \eta}\right)$ for some integer $s>1+d / 2$, such that $\|\hat{\rho}\|_{L^{\infty}} \leqslant r$, where $r$ is as in Proposition 1. Assume moreover that $\hat{\mathbf{u}}(0)$ is curl-free. Then there exists $C>0$ depending only on $r, s$ and $d$ such that

$$
\frac{d}{d T} E_{s}[\hat{\rho}, \hat{\mathbf{u}}] \leqslant C \eta\left(\left\|\left(\nabla_{X} \hat{\rho}, \nabla_{X} \hat{\mathbf{u}}\right)\right\|_{L^{\infty}}+\varepsilon\left\|D_{X}^{2} \hat{\rho}\right\|_{L^{\infty}}\right)\left(1+\eta \varepsilon\left\|\nabla_{X} \hat{\rho}\right\|_{L^{\infty}}\right) E_{s}[\hat{\rho}, \hat{\mathbf{u}}] .
$$

Proof. Let $0 \leqslant \sigma \leqslant s$ be given and $\alpha \in \mathbb{N}_{0}^{d}$ such that $|\alpha|=\sigma$. We work in the $X$ variable only, and use the simplified notations $\partial_{j}=\partial_{X_{j}}, \nabla=\nabla_{X}$. We recall that when $\hat{\mathbf{z}}$ is related to $(\hat{\rho}, \hat{\mathbf{u}})$ through (3), if the latter satisfies $\left(\mathrm{EK}_{\varepsilon, \eta}\right)$ then $(\rho=\varrho+\eta \hat{\rho}, \hat{\mathbf{z}})$ satisfies $\left(\operatorname{ES}_{\varepsilon, \eta}\right)$. Applying $\partial^{\alpha}$ to the second equation in $\left(\mathrm{ES}_{\varepsilon, \eta}\right)$, we obtain

$$
\begin{aligned}
\partial_{T} \partial^{\alpha} \hat{\mathbf{z}} & +\eta(\hat{\mathbf{u}} \cdot \nabla) \partial^{\alpha} \hat{\mathbf{z}}+i \eta\left(\nabla \partial^{\alpha} \hat{\mathbf{z}}\right) \hat{\mathbf{w}}+\frac{1}{\varepsilon} b(\rho) \partial^{\alpha} \hat{\mathbf{w}}+i \varepsilon \partial^{\alpha} \nabla(a(\rho) \nabla \cdot \hat{\mathbf{z}}) \\
& =\eta\left[\hat{\mathbf{u}} \cdot \nabla, \partial^{\alpha}\right] \hat{\mathbf{z}}+i \eta\left(\left(\nabla \partial^{\alpha} \hat{\mathbf{z}}\right) \hat{\mathbf{w}}-\partial^{\alpha}((\nabla \hat{\mathbf{z}}) \hat{\mathbf{w}})\right)+\frac{1}{\varepsilon}\left[b(\rho), \partial^{\alpha}\right] \hat{\mathbf{w}} \stackrel{\text { def }}{=} \mathbf{R} .
\end{aligned}
$$

Here above, the notation $[\cdot, \cdot]$ stands for a commutator, that is,

$$
\left[\partial^{\alpha}, \hat{\mathbf{u}} \cdot \nabla\right] \hat{\mathbf{z}} \stackrel{\text { def }}{=} \partial^{\alpha}((\hat{\mathbf{u}} \cdot \nabla) \hat{\mathbf{z}})-(\hat{\mathbf{u}} \cdot \nabla)\left(\partial^{\alpha} \hat{\mathbf{z}}\right), \quad\left[\partial^{\alpha}, b(\rho)\right] \hat{\mathbf{w}} \stackrel{\text { def }}{=} \partial^{\alpha}(b(\rho) \hat{\mathbf{w}})-b(\rho) \partial^{\alpha} \hat{\mathbf{w}} .
$$

All three commutators in the right-hand side $\mathbf{R}$ of (4) can be estimated by using the inequality (A.4) recalled in the appendix, and by noting in addition that $\left[\partial^{\alpha}, b(\rho)\right]=\left[\partial^{\alpha}, b(\rho)-b(\varrho)\right]$ (since $\varrho$ is constant), and, by definition of $\hat{\mathbf{w}}$ and since $s-1>d / 2$, that

$$
\|\hat{\mathbf{w}}\|_{H^{s-1}} \leqslant C(r) \varepsilon\|\nabla \hat{\rho}\|_{H^{s-1}} \leqslant C(r) \varepsilon\|\hat{\rho}\|_{H^{s}} \leqslant C(r) \varepsilon \sqrt{E_{s}[\hat{\rho}, \hat{\mathbf{z}}]}
$$

(by definition of $E_{s}$ ). We then infer that

$$
\begin{aligned}
\|\mathbf{R}\|_{L^{2}} & \leqslant C(r, s, d) \eta\left(\|\nabla \hat{\mathbf{z}}\|_{L^{\infty}}\|\hat{\mathbf{z}}\|_{H^{s}}+\frac{1}{\varepsilon}\|\hat{\rho}\|_{H^{s}}\|\hat{\mathbf{w}}\|_{L^{\infty}}+\frac{1}{\varepsilon}\|\nabla \hat{\rho}\|_{L^{\infty}}\|\hat{\mathbf{w}}\|_{H^{s-1}}\right) \\
& \leqslant C(r, s, d) \eta\|(\nabla \hat{\mathbf{z}}, \nabla \hat{\rho})\|_{L^{\infty}} \sqrt{E_{s}[\hat{\rho}, \hat{\mathbf{z}}]} .
\end{aligned}
$$

Here above and in what follows, $C(q)$ stands for a positive number depending only on $q$, whatever the quantity $q$. For convenience, the actual value of $C(q)$ may change from line to line. Therefore, using that

$$
\frac{\partial}{\partial T}\left(a^{\sigma}(\rho) \rho\right)+\eta \hat{\mathbf{u}} \cdot \nabla\left(a^{\sigma}(\rho) \rho\right)+\eta \rho \partial_{\rho}\left(\rho a^{\sigma}(\rho)\right) \nabla \cdot \hat{\mathbf{u}}=0
$$


by the first equation in $\left(\mathrm{ES}_{\varepsilon, \eta}\right)$, we obtain after integrations by parts that

$$
\begin{aligned}
& \frac{d}{d T} \int_{\mathbb{R}^{d}} \rho a^{\sigma}(\rho)\left|\partial^{\alpha} \hat{\mathbf{z}}\right|^{2} d X \leqslant \sum_{k=1}^{6} \mathcal{I}_{k}+C(r, s, d) \eta\|(\nabla \hat{\mathbf{z}}, \nabla \hat{\rho})\|_{L^{\infty}} E_{s}[\hat{\rho}, \hat{\mathbf{z}}] \\
& \mathcal{I}_{1} \stackrel{\text { def }}{=}-\eta \int_{\mathbb{R}^{d}}\left(\hat{\mathbf{u}} \cdot \nabla\left(\rho a^{\sigma}(\rho)\right)\right)\left|\partial^{\alpha} \hat{\mathbf{z}}\right|^{2} d X \\
& \mathcal{I}_{2} \stackrel{\text { def }}{=}-\eta \int_{\mathbb{R}^{d}} \rho \partial_{\rho}\left(\rho a^{\sigma}(\rho)\right)(\nabla \cdot \hat{\mathbf{u}})\left|\partial^{\alpha} \hat{\mathbf{z}}\right|^{2} d X \\
& \mathcal{I}_{3} \stackrel{\text { def }}{=} \eta \int_{\mathbb{R}^{d}}\left(\nabla \cdot\left(\rho a^{\sigma}(\rho) \hat{\mathbf{u}}\right)\right)\left|\partial^{\alpha} \hat{\mathbf{z}}\right|^{2} d X \\
& \mathcal{I}_{4} \stackrel{\text { def }}{=}-2 \eta \int_{\mathbb{R}^{d}} \rho a^{\sigma}(\rho)\left\langle i\left(\nabla\left(\partial^{\alpha} \hat{\mathbf{z}}\right)\right) \hat{\mathbf{w}}, \partial^{\alpha} \hat{\mathbf{z}}\right\rangle d X \\
& \mathcal{I}_{5} \stackrel{\text { def }}{=}-\frac{2}{\varepsilon} \int_{\mathbb{R}^{d}} \rho a^{\sigma}(\rho) b(\rho)\left\langle\partial^{\alpha} \hat{\mathbf{w}}, \partial^{\alpha} \hat{\mathbf{u}}\right\rangle d X \\
& \mathcal{I}_{6} \stackrel{\text { def }}{=} 2 \varepsilon \int_{\mathbb{R}^{d}} \rho a^{\sigma}(\rho)\left\langle i \partial^{\alpha} \hat{\mathbf{z}}, \partial^{\alpha}(\nabla(a(\rho) \nabla \cdot \hat{\mathbf{z}}))\right\rangle d X
\end{aligned}
$$

We can expand the divergence in $\mathcal{I}_{3}$ and notice that the term involving $\hat{\mathbf{u}} \cdot \nabla\left(\rho a^{\sigma}(\rho)\right)$ cancels out with $\mathcal{I}_{1}$. As a consequence,

$$
\begin{aligned}
\mathcal{I}_{1}+\mathcal{I}_{2}+\mathcal{I}_{3} & =\eta \int_{\mathbb{R}^{d}}\left(\rho a^{\sigma}(\rho)-\rho \partial_{\rho}\left(\rho a^{\sigma}(\rho)\right)\right)(\nabla \cdot \hat{\mathbf{u}})\left|\partial^{\alpha} \hat{\mathbf{z}}\right|^{2} d X \\
& =-\eta \int_{\mathbb{R}^{d}} \rho^{2} \partial_{\rho}\left(a^{\sigma}(\rho)\right)(\nabla \cdot \hat{\mathbf{u}})\left|\partial^{\alpha} \hat{\mathbf{z}}\right|^{2} d X \leqslant C(r, s, d) \eta\|\nabla \cdot \hat{\mathbf{u}}\|_{L^{\infty}} E_{s}[\hat{\rho}, \hat{\mathbf{z}}] .
\end{aligned}
$$

Concerning $\mathcal{I}_{6}$, an integration by parts and the Leibniz formula give

$$
\begin{aligned}
\mathcal{I}_{6}=-2 \varepsilon \int_{\mathbb{R}^{d}}\left\langle i \nabla \cdot\left(\rho a^{\sigma}(\rho) \partial^{\alpha} \hat{\mathbf{z}}\right), \partial^{\alpha}(a(\rho) \nabla \cdot \hat{\mathbf{z}})\right\rangle d X \\
=-2 \varepsilon \int_{\mathbb{R}^{d}}\left\langle i \rho a^{\sigma}(\rho) \nabla \cdot\left(\partial^{\alpha} \hat{\mathbf{z}}\right)+i\left(\nabla\left(\rho a^{\sigma}(\rho)\right)\right) \cdot \partial^{\alpha} \hat{\mathbf{z}},\right. \\
\left.a(\rho) \nabla \cdot\left(\partial^{\alpha} \hat{\mathbf{z}}\right)+\sum_{\substack{\beta \leqslant \alpha,|\beta|=\sigma-1}}\left(\begin{array}{c}
\alpha \\
\beta
\end{array}\right) \partial^{\alpha-\beta}(a(\rho)) \nabla \cdot\left(\partial^{\beta} \hat{\mathbf{z}}\right)+\mathbf{L}\right\rangle d X,
\end{aligned}
$$

where the lower order terms in $\mathbf{L}$ are such that

$$
\begin{aligned}
\varepsilon\|\nabla \mathbf{L}\|_{L^{2}} & \leqslant C(r, s, d) \eta\left(\|\hat{\mathbf{z}}\|_{H^{s}}\left\|\varepsilon D^{2} \hat{\rho}\right\|_{L^{\infty}}+\varepsilon\|\hat{\rho}\|_{H^{s+1}}\|\nabla \hat{\mathbf{z}}\|_{L^{\infty}}\right) \\
& \leqslant C(r, s, d) \eta\|(\nabla \hat{\rho}, \nabla \hat{\mathbf{z}})\|_{L^{\infty}} \sqrt{E_{s}[\hat{\rho}, \hat{\mathbf{z}}]} .
\end{aligned}
$$

We now expand the big inner product involved in $\mathcal{I}_{6}$, and notice that:

- the term $\left\langle i \rho a^{\sigma}(\rho) \nabla \cdot\left(\partial^{\alpha} \hat{\mathbf{z}}\right), a(\rho) \nabla \cdot\left(\partial^{\alpha} \hat{\mathbf{z}}\right)\right\rangle$ vanishes point wise (recall that $\langle\cdot, \cdot\rangle$ stands for a for real-valued inner product); 
- by (6) and an integration by parts, the contribution of $\mathbf{L}$ to $\mathcal{I}_{6}$ is bounded by

$$
\varepsilon C(r, s, d) \eta\|(\nabla \hat{\rho}, \nabla \hat{\mathbf{z}})\|_{L^{\infty}}\left(1+\eta\|\nabla \hat{\rho}\|_{L^{\infty}}\right) E_{s}[\hat{\rho}, \hat{\mathbf{z}}] ;
$$

- the contribution of derivatives of $\hat{\mathbf{z}}$ of order $\sigma$, coming from the inner product of the second term in the left factor and the sum on $\beta$ in the right factor of the integrand, is bounded by $\varepsilon C(r, s, d) \eta^{2}\|\nabla \hat{\rho}\|_{L^{\infty}}^{2}\|\hat{\mathbf{z}}\|_{H^{s}}^{2}$ by the Cauchy-Schwarz inequality.

This in turn gives

$$
\begin{aligned}
\mathcal{I}_{6} \leqslant & -2 \varepsilon \int_{\mathbb{R}^{d}}\left\langle i \rho a^{\sigma}(\rho) \nabla \cdot\left(\partial^{\alpha} \hat{\mathbf{z}}\right), \sum_{\substack{\beta \leqslant \alpha,|\beta|=\sigma-1}}\left(\begin{array}{c}
\alpha \\
\beta
\end{array}\right) \partial^{\alpha-\beta}(a(\rho)) \nabla \cdot\left(\partial^{\beta} \hat{\mathbf{z}}\right)\right\rangle d X \\
& +\mathcal{S}+C(r, s, d) \eta \varepsilon\|(\nabla \hat{\rho}, \nabla \hat{\mathbf{z}})\|_{L^{\infty}}\left(1+\eta\|\nabla \hat{\rho}\|_{L^{\infty}}\right) E_{s}[\hat{\rho}, \hat{\mathbf{z}}] \\
& \mathcal{S} \stackrel{\text { def }}{=}-2 \varepsilon \int_{\mathbb{R}^{d}}\left\langle i\left(\nabla\left(\rho a^{\sigma}(\rho)\right)\right) \cdot \partial^{\alpha} \hat{\mathbf{z}}, a(\rho) \nabla \cdot\left(\partial^{\alpha} \hat{\mathbf{z}}\right)\right\rangle d X
\end{aligned}
$$

By (3) we readily have that $\nabla\left(\rho a^{\sigma}(\rho)\right)=\frac{\eta \rho}{\varepsilon a(\rho)} \partial_{\rho}\left(\rho a^{\sigma}(\rho)\right) \hat{\mathbf{w}}$, and integrating by parts once more we see that

$$
\mathcal{S} \leqslant 2 \eta \int_{\mathbb{R}^{d}} \rho \partial_{\rho}\left(\rho a^{\sigma}(\rho)\right)\left\langle i \nabla\left(\partial^{\alpha} \hat{\mathbf{z}}\right) \hat{\mathbf{w}}, \partial^{\alpha} \hat{\mathbf{z}}\right\rangle d X+2 \eta\left\|\nabla\left(\rho \partial_{\rho}\left(\rho a^{\sigma}(\rho)\right) \hat{\mathbf{w}}\right)\right\|_{L^{\infty}} E_{s}[\hat{\rho}, \hat{\mathbf{z}}] .
$$

We now use that

$$
\begin{aligned}
\eta\left\|\nabla\left(\rho \partial_{\rho}\left(a^{\sigma}(\rho) \rho\right) \hat{\mathbf{w}}\right)\right\|_{L^{\infty}} & \leqslant C(r, s, d) \eta\left(\eta\|\hat{\mathbf{w}}\|_{L^{\infty}}\|\nabla \hat{\rho}\|_{L^{\infty}}+\|\nabla \hat{\mathbf{w}}\|_{L^{\infty}}\right) \\
& \leqslant C(r, s, d) \varepsilon \eta\left(\eta\|\nabla \hat{\rho}\|_{L^{\infty}}^{2}+\left\|D^{2} \hat{\rho}\right\|_{L^{\infty}}\right)
\end{aligned}
$$

to infer

$$
\mathcal{S} \leqslant 2 \eta \int_{\mathbb{R}^{d}} \rho \partial_{\rho}\left(\rho a^{\sigma}(\rho)\right)\left\langle i \nabla\left(\partial^{\alpha} \hat{\mathbf{z}}\right) \hat{\mathbf{w}}, \partial^{\alpha} \hat{\mathbf{z}}\right\rangle d X+C(r, s, d) \varepsilon \eta\left(\eta\|\nabla \hat{\rho}\|_{L^{\infty}}^{2}+\left\|D^{2} \hat{\rho}\right\|_{L^{\infty}}\right) E_{s}[\hat{\rho}, \hat{\mathbf{z}}] .
$$

Since $\partial_{\rho}\left(\rho a^{\sigma}(\rho)\right)=a^{\sigma}(\rho)+\rho \partial_{\rho}\left(a^{\sigma}(\rho)\right)$, the addition of

$$
\mathcal{I}_{4}=-2 \eta \int_{\mathbb{R}^{d}} a^{\sigma}(\rho) \rho\left\langle i\left(\nabla\left(\partial^{\alpha} \hat{\mathbf{z}}\right)\right) \hat{\mathbf{w}}, \partial^{\alpha} \hat{\mathbf{z}}\right\rangle d X
$$

to $\mathcal{I}_{6}$ cancels out the term involving $a^{\sigma}(\rho)$ in the bound found above for $\mathcal{S}$, so that

$$
\begin{aligned}
\mathcal{I}_{4}+\mathcal{I}_{6} \leqslant & C(r, s, d) \varepsilon \eta\left(\left\|\left(\nabla \hat{\rho}, \nabla \hat{\mathbf{z}}, D^{2} \hat{\rho}\right)\right\|_{L^{\infty}}+\eta\|(\nabla \hat{\rho}, \nabla \hat{\mathbf{z}})\|_{L^{\infty}}\|\nabla \hat{\rho}\|_{L^{\infty}}\right) E_{s}[\hat{\rho}, \hat{\mathbf{z}}] \\
& +\mathcal{K}+\sum_{\substack{\beta \leqslant \alpha,|\beta|=\sigma-1}} \mathcal{J}_{\beta}, \\
& \mathcal{J}_{\beta} \stackrel{\text { def }}{=}-2 \varepsilon \int_{\mathbb{R}^{d}} \rho a^{\sigma}(\rho)\left\langle i \nabla \cdot\left(\partial^{\alpha} \hat{\mathbf{z}}\right),\left(\begin{array}{c}
\alpha \\
\beta
\end{array}\right) \partial^{\alpha-\beta}(a(\rho)) \nabla \cdot\left(\partial^{\beta} \hat{\mathbf{z}}\right)\right\rangle d X \\
& \mathcal{K} \stackrel{\text { def }}{=} 2 \eta \int_{\mathbb{R}^{d}} \rho^{2} \partial_{\rho}\left(a^{\sigma}(\rho)\right)\left\langle i\left(\nabla \partial^{\alpha} \hat{\mathbf{z}}\right) \hat{\mathbf{w}}, \partial^{\alpha} \hat{\mathbf{z}}\right\rangle d X
\end{aligned}
$$


If, for any smooth enough mapping $\mathbf{Z}: \mathbb{R}^{d} \rightarrow \mathbb{C}^{d}$ we denote by curl $\mathbf{Z}$ the matrix-valued function defined by

$$
(\operatorname{curl} \mathbf{Z})_{j k}=\partial_{j} \mathbf{Z}_{k}-\partial_{k} \mathbf{Z}_{j}
$$

we see that for $\mathbf{W}, \mathbf{Y}$ any other smooth enough mappings $\mathbb{R}^{d} \rightarrow \mathbb{C}^{d}$,

$$
\langle(\nabla \mathbf{Z}) \mathbf{W}, \mathbf{Y}\rangle=\langle(\mathbf{W} \cdot \nabla) \mathbf{Z}, \mathbf{Y}\rangle+\langle(\operatorname{curl} \mathbf{Z}) \mathbf{W}, \mathbf{Y}\rangle .
$$

In particular, we can write

$$
\begin{aligned}
\mathcal{K}= & 2 \sigma \eta \int_{\mathbb{R}^{d}} \rho^{2} a^{\sigma-1}(\rho) a^{\prime}(\rho)\left\langle i \hat{\mathbf{w}} \cdot \nabla \partial^{\alpha} \hat{\mathbf{z}}, \partial^{\alpha} \hat{\mathbf{z}}\right\rangle d X \\
& +2 \sigma \eta \int_{\mathbb{R}^{d}} \rho^{2} a^{\sigma-1}(\rho) a^{\prime}(\rho)\left\langle i\left(\partial^{\alpha} \operatorname{curl} \hat{\mathbf{z}}\right) \hat{\mathbf{w}}, \partial^{\alpha} \hat{\mathbf{z}}\right\rangle d X
\end{aligned}
$$

On the other hand, using that $\partial^{\alpha-\beta}(a(\rho))=a^{\prime}(\rho) \partial^{\alpha-\beta} \rho$ when $\alpha-\beta$ has length one, we have

$$
\mathcal{J}_{\beta}=-2 \varepsilon \int_{\mathbb{R}^{d}} \rho a^{\sigma}(\rho) a^{\prime}(\rho)\left(\partial^{\alpha-\beta} \rho\right)\left\langle i \nabla \cdot\left(\partial^{\alpha} \hat{\mathbf{z}}\right), \nabla \cdot\left(\partial^{\beta} \hat{\mathbf{z}}\right)\right\rangle d X,
$$

which gives, after integrating by parts and using A.4,

$$
\begin{aligned}
\mathcal{J}_{\beta} \leqslant & 2 \varepsilon \int_{\mathbb{R}^{d}} \rho a^{\sigma}(\rho) a^{\prime}(\rho)\left(\partial^{\alpha-\beta} \rho\right)\left\langle i \partial^{\alpha} \hat{\mathbf{z}}, \nabla\left(\nabla \cdot\left(\partial^{\beta} \hat{\mathbf{z}}\right)\right)\right\rangle d X \\
& +C(r, s, d) \varepsilon\left\|\nabla\left(\rho a^{\sigma}(\rho) a^{\prime}(\rho) \partial^{\alpha-\beta} \rho\right)\right\|_{L^{\infty}} E_{s}[\hat{\rho}, \hat{\mathbf{z}}] .
\end{aligned}
$$

Now, observing that for any smooth enough mappings $\mathbf{Z}, \mathbf{Y}: \mathbb{R}^{d} \rightarrow \mathbb{C}^{d}$,

$$
\langle\mathbf{Z}, \nabla(\nabla \cdot \mathbf{Y})\rangle=\langle\mathbf{Z}, \Delta \mathbf{Y}\rangle+\langle\mathbf{Z}, \nabla \cdot(\operatorname{curl} \mathbf{Y})\rangle,
$$

(where we have used the notation $\nabla \cdot \mathbf{M}$ for the vector field defined by $(\nabla \cdot \mathbf{M})_{j}=\sum_{k=1}^{d} \partial_{k} \mathbf{M}_{j k}$, associated with the matrix-valued function $\mathbf{M}=\operatorname{curl} \mathbf{Y}$ ), we find that

$$
\begin{aligned}
\mathcal{J}_{\beta} \leqslant & C(r, s, d) \varepsilon \eta\left(\eta\|\nabla \hat{\rho}\|_{L^{\infty}}^{2}+\left\|D^{2} \hat{\rho}\right\|_{L^{\infty}}\right) E_{s}[\hat{\rho}, \hat{\mathbf{z}}]+2 \varepsilon \int_{\mathbb{R}^{d}} \rho a^{\sigma}(\rho) a^{\prime}(\rho)\left(\partial^{\alpha-\beta} \rho\right)\left\langle i \partial^{\alpha} \hat{\mathbf{z}}, \Delta \partial^{\beta} \hat{\mathbf{z}}\right\rangle d X \\
& +2 \varepsilon \int_{\mathbb{R}^{d}} \rho a^{\sigma}(\rho) a^{\prime}(\rho)\left(\partial^{\alpha-\beta} \rho\right)\left\langle i \partial^{\alpha} \hat{\mathbf{z}}, \nabla \cdot\left(\partial^{\beta} \operatorname{curl} \hat{\mathbf{z}}\right)\right\rangle d X
\end{aligned}
$$

To finish with the estimate of $\mathcal{J}_{\beta}$, we integrate by parts again, and arrive at

$$
\begin{aligned}
\mathcal{J}_{\beta} \leqslant & C(r, s, d) \varepsilon \eta\left(\eta\|\nabla \hat{\rho}\|_{L^{\infty}}^{2}+\left\|D^{2} \hat{\rho}\right\|_{L^{\infty}}\right) E_{s}[\hat{\rho}, \hat{\mathbf{z}}] \\
& -2 \varepsilon \int_{\mathbb{R}^{d}} \rho a^{\sigma}(\rho) a^{\prime}(\rho)\left(\partial^{\alpha-\beta} \rho\right) \sum_{j=1}^{d}\left\langle i \partial^{\alpha-\beta} \partial_{j} \partial^{\beta} \hat{\mathbf{z}}, \partial_{j} \partial^{\beta} \hat{\mathbf{z}}\right\rangle d X \\
& -2 \varepsilon \int_{\mathbb{R}^{d}} \rho a^{\sigma}(\rho) a^{\prime}(\rho)\left(\partial^{\alpha-\beta} \rho\right)\left\langle i \partial^{\alpha} D \hat{\mathbf{z}}, \partial^{\beta} \operatorname{curl} \hat{\mathbf{z}}\right\rangle_{\mathcal{M}_{d}(\mathbb{C})} d X
\end{aligned}
$$


where $\langle A, B\rangle_{\mathcal{M}_{d}(\mathbb{C})} \stackrel{\text { def }}{=} \operatorname{Re}\left(\operatorname{Tr}\left(A B^{*}\right)\right)$ is the usual real inner product on $\mathcal{M}_{d}(\mathbb{C})$, and $D \hat{\mathbf{z}} \stackrel{\text { def }}{=}$ $(\nabla \hat{\mathbf{z}})^{\mathrm{T}}$.

The remaining term $\mathcal{I}_{5}$ will turn out to cancel out, up to a remainder term, with the time derivative of $\int_{\mathbb{R}^{d}} g^{\prime}(\rho) a^{\sigma}(\rho)\left(\partial^{\alpha} \hat{\rho}\right)^{2} d X$. In order to see this, we differentiate the first equation in $\left(\mathrm{ES}_{\varepsilon, \eta}\right)$ and obtain

$$
\partial_{T} \partial^{\alpha} \hat{\rho}+\eta(\hat{\mathbf{u}} \cdot \nabla) \partial^{\alpha} \hat{\rho}+\rho \nabla \cdot \partial^{\alpha} \hat{\mathbf{u}}=-\eta\left[\partial^{\alpha}, \hat{\mathbf{u}} \cdot \nabla\right] \hat{\rho}-\left[\partial^{\alpha}, \rho \nabla \cdot\right] \hat{\mathbf{u}} .
$$

By (A.4), the commutators in the right-hand side here above have an $L^{2}$ norm bounded by

$$
C(r, s, d) \eta\|(\nabla \hat{\rho}, \nabla \hat{\mathbf{u}})\|_{L^{\infty}} \sqrt{E_{s}[\hat{\rho}, \hat{\mathbf{z}}]}
$$

Furthermore, by the first equation in $\left(\mathrm{ES}_{\varepsilon, \eta}\right)$ again, we have

$$
\partial_{T}\left(g^{\prime}(\rho) a^{\sigma}(\rho)\right)+\eta \hat{\mathbf{u}} \cdot \nabla\left(g^{\prime}(\rho) a^{\sigma}(\rho)\right)+\eta \rho \partial_{\rho}\left(g^{\prime}(\rho) a^{\sigma}(\rho)\right) \nabla \cdot \hat{\mathbf{u}}=0 .
$$

Arguing as for $\mathcal{I}_{1}+\mathcal{I}_{2}+\mathcal{I}_{3}$, we thus find that

$$
\begin{aligned}
\frac{d}{d T} \int_{\mathbb{R}^{d}} g^{\prime}(\rho) a^{\sigma}(\rho)\left(\partial^{\alpha} \hat{\rho}\right)^{2} d X \leqslant & C(r, s, d) \eta\|(\nabla \hat{\rho}, \nabla \hat{\mathbf{u}})\|_{L^{\infty}} E_{s}[\hat{\rho}, \hat{\mathbf{z}}] \\
& -\int_{\mathbb{R}^{d}} 2 \rho g^{\prime}(\rho) a^{\sigma}(\rho) \partial^{\alpha} \hat{\rho} \nabla \cdot\left(\partial^{\alpha} \hat{\mathbf{u}}\right) d X
\end{aligned}
$$

Integrating by parts, using again that

$$
\rho \hat{\mathbf{w}}=\varepsilon a(\rho) \nabla \hat{\rho}, \quad\|\hat{\mathbf{w}}\|_{H^{s-1}} \leqslant C_{s, d} \varepsilon \sqrt{E_{s}[\hat{\rho}, \hat{\mathbf{z}}]}
$$

and combining this with (A.4), we arrive at

$$
\begin{aligned}
\frac{d}{d T} \int_{\mathbb{R}^{d}} g^{\prime}(\rho) a^{\sigma}(\rho)\left(\partial^{\alpha} \hat{\rho}\right)^{2} d X \leqslant & C(r, s, d) \eta\|(\nabla \hat{\rho}, \nabla \hat{\mathbf{u}})\|_{L^{\infty}} E_{s}[\hat{\rho}, \hat{\mathbf{z}}] \\
& +\frac{2}{\varepsilon} \int_{\mathbb{R}^{d}} \rho \frac{\rho g^{\prime}(\rho)}{a(\rho)} a^{\sigma}(\rho)\left\langle\partial^{\alpha} \hat{\mathbf{w}}, \partial^{\alpha} \hat{\mathbf{u}}\right\rangle d X .
\end{aligned}
$$

Since $a(\rho) b(\rho)=\rho g^{\prime}(\rho)$, the integral in the right-hand side of $(10)$ here above cancels out with the integral $\mathcal{I}_{5}$ in (5). Therefore, using (8) and (9) in (7), and combining (10) with (5), 
we obtain

$$
\begin{aligned}
& \frac{d}{d T} \int_{\mathbb{R}^{d}} \rho a^{\sigma}(\rho)\left|\partial^{\alpha} \hat{\mathbf{z}}\right|^{2}+g^{\prime}(\rho) a^{\sigma}(\rho)\left(\partial^{\alpha} \hat{\rho}\right)^{2} d X \\
& \leqslant \\
& \quad C(r, s, d) \eta\left(\|(\nabla \hat{\rho}, \nabla \hat{\mathbf{z}})\|_{L^{\infty}}\left(1+\eta \varepsilon\|\nabla \hat{\rho}\|_{L^{\infty}}\right)+\varepsilon\left\|D^{2} \hat{\rho}\right\|_{L^{\infty}}\right) E_{s}[\hat{\rho}, \hat{\mathbf{z}}] \\
& \quad+2 \sigma \eta \int_{\mathbb{R}^{d}} \rho^{2} a^{\sigma-1}(\rho) a^{\prime}(\rho)\left\langle i \hat{\mathbf{w}} \cdot \nabla \partial^{\alpha} \hat{\mathbf{z}}, \partial^{\alpha} \hat{\mathbf{z}}\right\rangle d X \\
& \quad+2 \sigma \eta \int_{\mathbb{R}^{d}} \rho^{2} a^{\sigma-1}(\rho) a^{\prime}(\rho)\left\langle i\left(\partial^{\alpha} \operatorname{curl} \hat{\mathbf{z}}\right) \hat{\mathbf{w}}, \partial^{\alpha} \hat{\mathbf{z}}\right\rangle d X \\
& \quad-2 \sum_{\substack{\beta \leqslant \alpha,|\beta|=\sigma-1}} \varepsilon\left(\begin{array}{c}
\alpha \\
\beta
\end{array}\right) \int_{\mathbb{R}^{d}} \rho a^{\sigma}(\rho) a^{\prime}(\rho)\left(\partial^{\alpha-\beta} \rho\right) \sum_{j=1}^{d}\left\langle i \partial^{\alpha-\beta} \partial_{j} \partial^{\beta} \hat{\mathbf{z}}, \partial_{j} \partial^{\beta} \hat{\mathbf{z}}\right\rangle d X \\
& \quad-2 \sum_{\substack{\beta \leqslant \alpha,|\beta|=\sigma-1}} \varepsilon\left(\begin{array}{c}
\alpha \\
\beta
\end{array}\right) \int_{\mathbb{R}^{d}} \rho a^{\sigma}(\rho) a^{\prime}(\rho)\left(\partial^{\alpha-\beta} \rho\right)\left\langle i \partial^{\alpha} D \hat{\mathbf{z}}, \partial^{\beta} \operatorname{curl} \hat{\mathbf{z}}\right\rangle_{\mathcal{M}_{d}(\mathbb{C})} d X .
\end{aligned}
$$

At this stage, we use the hypothesis that $\hat{\mathbf{z}}$ is a gradient vector field, so that the two terms involving the curl operator in (11) cancel out. Summing over $\alpha$ with $|\alpha|=\sigma$ then gives

$$
\begin{aligned}
\frac{d}{d T} \dot{E}_{\sigma}(\hat{\rho}, \hat{\mathbf{z}}) \leqslant & C(r, s, d) \eta\|(\nabla \hat{\rho}, \nabla \hat{\mathbf{z}})\|_{L^{\infty}}\left(1+\eta \varepsilon\|\nabla \hat{\rho}\|_{L^{\infty}}\right) E_{s}[\hat{\rho}, \hat{\mathbf{z}}] \\
& +2 \eta \sigma ! \sum_{|\alpha|=\sigma} \frac{\sigma}{\alpha !} \int_{\mathbb{R}^{d}} \rho^{2} a^{\sigma-1}(\rho) a^{\prime}(\rho)\left\langle i \hat{\mathbf{w}} \cdot \nabla \partial^{\alpha} \hat{\mathbf{z}}, \partial^{\alpha} \hat{\mathbf{z}}\right\rangle d X \\
& -2 \sigma ! \sum_{|\alpha|=\sigma} \sum_{\substack{\beta \leqslant \alpha,|\beta|=\sigma-1}} \frac{\varepsilon}{\alpha !}\left(\begin{array}{c}
\alpha \\
\beta
\end{array}\right) \int_{\mathbb{R}^{d}} \rho a^{\sigma}(\rho) a^{\prime}(\rho)\left(\partial^{\alpha-\beta} \rho\right) \sum_{j=1}^{d}\left\langle i \partial^{\alpha-\beta} \partial_{j} \partial^{\beta} \hat{\mathbf{z}}, \partial_{j} \partial^{\beta} \hat{\mathbf{z}}\right\rangle d X
\end{aligned}
$$

In the double sum, there holds $\frac{1}{\alpha !}\left(\begin{array}{c}\alpha \\ \beta\end{array}\right)=\frac{1}{\beta !}$, since $\alpha-\beta$ has length one. Exchanging the order of summation on $\alpha$ and $\beta$, then summing at fixed $\alpha^{\prime}=\beta+e_{j}$, and using again that $\varepsilon a(\rho) \partial_{k} \rho=\eta \rho \hat{\mathbf{w}}_{k}$, we can rewrite this double sum as

$$
\begin{aligned}
& \varepsilon \sum_{|\beta|=\sigma-1} \frac{1}{\beta !} \sum_{k=1}^{d} \int_{\mathbb{R}^{d}} \rho a^{\sigma}(\rho) a^{\prime}(\rho) \partial_{k} \rho \sum_{j=1}^{d}\left\langle i \partial_{k} \partial_{j} \partial^{\beta} \hat{\mathbf{z}}, \partial_{j} \partial^{\beta} \hat{\mathbf{z}}\right\rangle d X \\
& =\eta \sum_{|\beta|=\sigma-1} \frac{1}{\beta !} \int_{\mathbb{R}^{d}} \rho^{2} a^{\sigma-1}(\rho) a^{\prime}(\rho) \sum_{j=1}^{d}\left\langle i \hat{\mathbf{w}} \cdot \nabla \partial_{j} \partial^{\beta} \hat{\mathbf{z}}, \partial_{j} \partial^{\beta} \hat{\mathbf{z}}\right\rangle d X \\
& =\eta \sum_{\left|\alpha^{\prime}\right|=\sigma} \sum_{j=1}^{d} \frac{\alpha_{j}^{\prime}}{\left(\alpha^{\prime}\right) !} \int_{\mathbb{R}^{d}} \rho^{2} a^{\sigma-1}(\rho) a^{\prime}(\rho)\left\langle i \hat{\mathbf{w}} \cdot \nabla \partial^{\alpha^{\prime}} \hat{\mathbf{z}}, \partial^{\alpha^{\prime}} \hat{\mathbf{z}}\right\rangle d X \\
& =\eta \sigma \sum_{\left|\alpha^{\prime}\right|=\sigma} \frac{1}{\left(\alpha^{\prime}\right) !} \int_{\mathbb{R}^{d}} \rho^{2} a^{\sigma-1}(\rho) a^{\prime}(\rho)\left\langle i \hat{\mathbf{w}} \cdot \nabla \partial^{\alpha^{\prime}} \hat{\mathbf{z}}, \partial^{\alpha^{\prime}} \hat{\mathbf{z}}\right\rangle d X
\end{aligned}
$$


since the integral does not depend on $j$ and $\sum_{j} \alpha_{j}^{\prime}=\sigma$. Therefore, the two sums in (12) cancel out (this is due to the coefficients $1 / \alpha$ ! in the definition of $\dot{E}_{\sigma}$ ). The conclusion then follows by summation over $\sigma$.

\subsection{Proof of uniform bounds in the general case}

In this section, $s$ is any real number greater than $1+d / 2$. Our aim is to prove Theorem 2 in the general case. As we have seen in the a priori estimates above, there remain some 'bad' terms when the velocity field $\mathbf{u}$ is not potential. This is why, as in [4, the solenoidal and potential parts of $\mathbf{u}$ require different weights. In fact, our proof of Theorem 2 will parallel very closely the proof of Proposition 3.4 in [4], except that we pay attention to the parameters $(\eta, \varepsilon)$, and insert the contribution of the nonlinear function $g^{\prime}(\rho)$.

As in the proof of Proposition $4, \nabla$ stands for $\nabla_{X}$ in what follows. As a preliminary step, we rewrite the second equation in $\left(\mathrm{ES}_{\varepsilon, \eta}\right)$ as an equation for $\hat{\mathbf{Z}} \stackrel{\text { def }}{=} \sqrt{\rho} \hat{\mathbf{z}}$ instead of $\hat{\mathbf{z}}$. Using that $\rho \eta \hat{\mathbf{w}}=\varepsilon a(\rho) \nabla \rho$ (which is just a reformulation of (3p), the first order term $i \eta(\nabla \hat{\mathbf{z}}) \hat{\mathbf{w}}$ can combined with the second order one $i \varepsilon \nabla(a(\rho) \nabla \cdot \hat{\mathbf{z}})$ to obtain

$$
\begin{array}{r}
\partial_{T} \hat{\mathbf{Z}}+\eta(\hat{\mathbf{u}} \cdot \nabla) \hat{\mathbf{Z}}+\frac{1}{\varepsilon} b(\rho) \hat{\mathbf{w}}+i \varepsilon \nabla(a(\rho) \nabla \cdot \hat{\mathbf{Z}})+i \varepsilon a(\rho)\left(\nabla_{0} \hat{\mathbf{Z}}\right) \nabla \log \sqrt{\rho}= \\
-\frac{1}{2} \eta(\nabla \cdot \hat{\mathbf{u}}) \hat{\mathbf{Z}}+i \varepsilon \nabla(a(\rho) \nabla \sqrt{\rho}) \frac{\hat{\mathbf{Z}}}{\sqrt{\rho}},
\end{array}
$$

where the operator $\nabla_{0}$ is defined by

$$
\left(\nabla_{0} \mathbf{Z}\right)_{j k}=\partial_{j} Z_{k}-(\nabla \cdot \mathbf{Z}) \delta_{j k}, \quad \text { or equivalently, } \nabla_{0} \mathbf{Z} \stackrel{\text { def }}{=} \nabla \mathbf{Z}-(\nabla \cdot \mathbf{Z}) \mathbf{I} .
$$

The advantage of this formulation is that it trivializes the proof of zeroth order estimates (Proposition 2), since

$$
\int_{\mathbb{R}^{d}}\left\langle i\left(\nabla_{0} \hat{\mathbf{Z}}\right) \mathbf{W}, \hat{\mathbf{Z}}\right\rangle=0
$$

for all potential vector fields $\mathbf{W}$, and in particular for $\mathbf{W}=a(\rho) \nabla \log \sqrt{\rho}$. The idea is to keep this nice structure for higher order derivatives, which means writing equations for $\hat{\mathbf{Z}}^{s}:=\sqrt{\rho} \Lambda^{s} \hat{\mathbf{z}}$ instead of $\Lambda^{s} \hat{\mathbf{z}}$, where $\Lambda^{s}$ denotes the Fourier multiplier operator

$$
\Lambda^{s} \stackrel{\text { def }}{=}(1-\Delta)^{s / 2} .
$$

However, we have to cope with a 'bad' commutator, namely in $\nabla\left[a(\rho), \Lambda^{s}\right] \nabla \cdot$, which already appears in the equation for $\Lambda^{s} \hat{\mathbf{z}}$. Pointing out its principal part, we can write as in [4]

$$
\begin{aligned}
\nabla\left[a(\rho), \Lambda^{s}\right] \nabla \cdot \hat{\mathbf{z}} & \stackrel{\text { def }}{=} \mathbf{R}_{0}+s \nabla\left(\nabla a(\rho) \cdot \Lambda^{s-2} \nabla(\nabla \cdot \hat{\mathbf{z}})\right) \\
& \stackrel{\text { def }}{=} \mathbf{R}_{0}+\mathbf{R}_{00}-s \nabla a(\rho) \cdot \nabla\left(\mathcal{Q} \Lambda^{s} \hat{\mathbf{z}}\right),
\end{aligned}
$$

where

$$
\left\|\mathbf{R}_{0}\right\|_{L^{2}} \lesssim\left\|D^{2} a(\rho)\right\|_{H^{s-1}}\|\nabla \cdot \hat{\mathbf{z}}\|_{L^{\infty}}+\left\|D^{2} a(\rho)\right\|_{L^{\infty}}\|\nabla \cdot \hat{\mathbf{z}}\|_{H^{s-1}}
$$




$$
\left\|\mathbf{R}_{00}\right\|_{L^{2}} \lesssim\|\nabla a(\rho)\|_{W^{1, \infty}}\|\hat{\mathbf{z}}\|_{H^{s}}
$$

and $\mathcal{Q}$ is the $L^{2}$-orthogonal projector onto potential vector fields. Consequently, by applying $\Lambda^{s}$ to the second equation in $\left(\mathrm{ES}_{\varepsilon, \eta}\right)$, multiplying by $\sqrt{\rho}$, and using also the first equation in $\left(\mathrm{ES}_{\varepsilon, \eta}\right)$, we see that

$$
\begin{array}{r}
\partial_{T} \hat{\mathbf{Z}}^{s}+\eta(\hat{\mathbf{u}} \cdot \nabla) \hat{\mathbf{Z}}^{s}+\frac{1}{\varepsilon} b(\rho) \sqrt{\rho} \Lambda^{s} \hat{\mathbf{w}}+i \varepsilon \nabla\left(a(\rho) \nabla \cdot \hat{\mathbf{Z}}^{s}\right)+ \\
+i \varepsilon a(\rho)\left(\nabla_{0} \hat{\mathbf{Z}}^{s}\right) \nabla \log \sqrt{\rho}+i \varepsilon s \sqrt{\rho} \nabla a(\rho) \cdot \nabla\left(\mathcal{Q} \Lambda^{s} \hat{\mathbf{Z}}\right)= \\
-\frac{1}{2} \eta(\nabla \cdot \hat{\mathbf{u}}) \hat{\mathbf{Z}}^{s}+i \varepsilon \nabla(a(\rho) \nabla \sqrt{\rho}) \frac{\hat{\mathbf{Z}}^{s}}{\sqrt{\rho}}+i \varepsilon \sqrt{\rho}\left(\mathbf{R}_{0}+\mathbf{R}_{00}\right)+\sqrt{\rho} \mathbf{R}
\end{array}
$$

with

$$
\mathbf{R} \stackrel{\text { def }}{=} \eta\left[\hat{\mathbf{u}} \cdot \nabla, \Lambda^{s}\right] \hat{\mathbf{z}}+i \eta\left(\left(\nabla \Lambda^{s} \hat{\mathbf{z}}\right) \hat{\mathbf{w}}-\Lambda^{s}((\nabla \hat{\mathbf{z}}) \hat{\mathbf{w}})\right)+\frac{1}{\varepsilon}\left[b(\rho), \Lambda^{s}\right] \hat{\mathbf{w}}
$$

being bounded as in the proof of Proposition 4 by

$$
\|\mathbf{R}\|_{L^{2}} \leqslant C(r, s, d) \eta\|(\nabla \hat{\mathbf{z}}, \nabla \hat{\rho})\|_{L^{\infty}}\|(\hat{\rho}, \hat{\mathbf{z}})\|_{H^{s}},
$$

and also

$$
\left\|i \varepsilon\left(\mathbf{R}_{0}+\mathbf{R}_{00}\right)\right\|_{L^{2}} \leqslant C(r, s, d) \eta\|(\nabla \hat{\mathbf{z}}, \nabla \hat{\rho})\|_{L^{\infty}}\|(\hat{\rho}, \hat{\mathbf{z}})\|_{H^{s}}
$$

by the estimates mentioned above and the fact that $\varepsilon\|\hat{\rho}\|_{H^{s+1}} \leqslant C(r, s, d)\|(\hat{\rho}, \hat{\mathbf{z}})\|_{H^{s}}$. Therefore, apart form the term $\varepsilon^{-1} b(\rho) \sqrt{\rho} \Lambda^{s} \hat{\mathbf{w}}$ that we will deal with afterwards, the only troublesome term regarding the time derivative of $\left\|\hat{\mathbf{Z}}^{s}\right\|_{L^{2}}$ is the one involving $\nabla\left(\mathcal{Q} \Lambda^{s} \hat{\mathbf{z}}\right)$, which corresponds to derivatives of order $s+1$. This is where the use of an appropriate weight comes into play. In fact, whatever the positive-valued weight (or gauge) $\psi=\psi(\rho)$, the equation above for $\hat{\mathbf{Z}}^{s}$ and the first equation in $\left(\mathrm{ES}_{\varepsilon, \eta}\right)$ give, after some manipulations,

$$
\begin{array}{r}
\partial_{T} \hat{\mathbf{Y}}^{s}+\eta(\hat{\mathbf{u}} \cdot \nabla) \hat{\mathbf{Y}}^{s}+\frac{1}{\varepsilon} b(\rho) \sqrt{\rho} \psi(\rho) \Lambda^{s} \hat{\mathbf{w}}+i \varepsilon \nabla\left(a(\rho) \nabla \cdot \hat{\mathbf{Y}}^{s}\right)+ \\
+i \varepsilon a(\rho)\left(\nabla_{0} \hat{\mathbf{Y}}^{s}\right) \nabla \log \left(\frac{\sqrt{\rho} a^{s}(\rho)}{\psi(\rho)}\right) \\
-i \varepsilon s \sqrt{\rho} \psi(\rho)\left(\nabla \mathcal{P} \Lambda^{s} \hat{\mathbf{z}}\right) \nabla a(\rho)+i \varepsilon a(\rho)\left(\nabla \cdot \hat{\mathbf{Y}}^{s}\right) \nabla \log \left(\frac{a^{s}(\rho)}{\psi^{2}(\rho)}\right)= \\
-\frac{1}{2} \eta(\nabla \cdot \hat{\mathbf{u}})\left(1+\rho \partial_{\rho} \log \left(\psi^{2}(\rho)\right)\right) \hat{\mathbf{Y}}^{s} \\
+i \varepsilon\left(\frac{\nabla(a(\rho) \nabla \sqrt{\rho})}{\sqrt{\rho}}+\frac{\nabla(a(\rho) \nabla \psi(\rho))}{\psi(\rho)}+a(\rho)(\nabla \log \psi(\rho)) \otimes \nabla \log \left(\frac{a^{s}(\rho)}{\psi(\rho)}\right)\right) \hat{\mathbf{Y}}^{s} \\
+i \varepsilon \sqrt{\rho} \psi(\rho)\left(\mathbf{R}_{0}+\mathbf{R}_{00}\right)+\sqrt{\rho} \psi(\rho) \mathbf{R}
\end{array}
$$

with $\hat{\mathbf{Y}}^{s} \stackrel{\text { def }}{=} \psi(\rho) \hat{\mathbf{Z}}^{s}=\sqrt{\rho} \psi(\rho) \Lambda^{s} \hat{\mathbf{Z}}$, and $\mathcal{P} \stackrel{\text { def }}{=} I-\mathcal{Q}$. From this expression and previous estimates, we see that the loss of spatial derivatives in the time derivative of $\left\|\hat{\mathbf{Y}}^{s}\right\|_{L^{2}}$ is only due to the terms in the third row. Of course, these terms vanish when $\hat{\mathbf{u}}$, and thus also 
$\hat{\mathbf{z}}$, is potential (hence $\mathcal{P} \Lambda^{s} \hat{\mathbf{z}}=\Lambda^{s} \mathcal{P} \hat{\mathbf{z}}=0$ ), if we choose $\psi^{2}(\rho)=a^{s}(\rho)$. Provided that the term $\varepsilon^{-1} b(\rho) \sqrt{\rho} \Lambda^{s} \hat{\mathbf{w}}$ is properly handled, this gives a shorter proof, compared to that of Proposition 4, of uniform bounds in the potential case. In the general case, the idea is to estimate separately $\left\|\mathcal{Q} \hat{\mathbf{Y}}^{s}\right\|_{L^{2}}$ and $\left\|\mathcal{P} \hat{\mathbf{X}}^{s}\right\|_{L^{2}}$ where $\hat{\mathbf{X}}^{s} \stackrel{\text { def }}{=} \varphi(\rho) \hat{\mathbf{Z}}^{s}=\sqrt{\rho} \varphi(\rho) \Lambda^{s} \hat{\mathbf{z}}$ for some other weight $\varphi$. These estimates will be based, as in [4], on a preliminary observation using only integration by parts and the properties $\nabla \cdot \mathcal{P} \equiv 0, \operatorname{curl} \mathcal{Q} \equiv 0$, which gives that

$$
\begin{array}{r}
\frac{d}{d T} \frac{1}{2}\left\|\mathcal{Q} \hat{\mathbf{Y}}^{s}\right\|_{L^{2}}^{2}=\int_{\mathbb{R}^{d}}\left\langle\mathcal{Q} \hat{\mathbf{Y}}^{s},\left(\partial_{t}+\eta \hat{\mathbf{u}} \cdot \nabla\right) \hat{\mathbf{Y}}^{s}\right\rangle d X+\eta \int_{\mathbb{R}^{d}}(\nabla \cdot \hat{\mathbf{u}})\left\langle\mathcal{Q} \hat{\mathbf{Y}}^{s}, \frac{1}{2} \mathcal{Q} \hat{\mathbf{Y}}^{s}+\mathcal{P} \hat{\mathbf{Y}}^{s}\right\rangle d X \\
-\eta \int_{\mathbb{R}^{d}}\left\langle\mathcal{P} \hat{\mathbf{Y}}^{s},(\nabla \hat{\mathbf{u}}) \mathcal{Q} \hat{\mathbf{Y}}^{s}\right\rangle d X \\
\frac{d}{d T} \frac{1}{2}\left\|\mathcal{P} \hat{\mathbf{X}}^{s}\right\|_{L^{2}}^{2}=\int_{\mathbb{R}^{d}}\left\langle\mathcal{P} \hat{\mathbf{X}}^{s},\left(\partial_{t}+\eta \hat{\mathbf{u}} \cdot \nabla\right) \hat{\mathbf{X}}^{s}\right\rangle d X+\eta \int_{\mathbb{R}^{d}}(\nabla \cdot \hat{\mathbf{u}}) \frac{1}{2}\left\langle\mathcal{P} \hat{\mathbf{X}}^{s}, \mathcal{P} \hat{\mathbf{X}}^{s}\right\rangle d X \\
+\eta \int_{\mathbb{R}^{d}}\left\langle\mathcal{P} \hat{\mathbf{X}}^{s},(\nabla \hat{\mathbf{u}}) \mathcal{Q} \hat{\mathbf{X}}^{s}\right\rangle d X
\end{array}
$$

Using the equations satisfied by $\hat{\mathbf{Y}}^{s}$ and $\hat{\mathbf{X}}^{s}$ - the latter being identical to the former if we substitute $\hat{\mathbf{X}}^{s}$ for $\hat{\mathbf{Y}}^{s}$ and $\varphi$ for $\psi$-, and summing the equations here above, we are left with harmless remainder terms, bounded in by $C(r, s, d) \eta\|(\nabla \hat{\mathbf{z}}, \nabla \hat{\rho})\|_{L^{\infty}}\|(\hat{\rho}, \hat{\mathbf{z}})\|_{H^{s}}^{2}$, plus a number of terms that must be handled carefully. Among these delicate terms is

$$
\mathcal{I} \stackrel{\text { def }}{=}-\frac{1}{\varepsilon} \int_{\mathbb{R}^{d}}\left\langle\mathcal{Q} \hat{\mathbf{Y}}^{s}, b(\rho) \sqrt{\rho} \psi(\rho) \Lambda^{s} \hat{\mathbf{w}}\right\rangle+\left\langle\mathcal{P} \hat{\mathbf{X}}^{s}, b(\rho) \sqrt{\rho} \varphi(\rho) \Lambda^{s} \hat{\mathbf{w}}\right\rangle d X
$$

Noting that both $\varepsilon^{-1} b(\rho) \sqrt{\rho} \psi(\rho) \Lambda^{s} \hat{\mathbf{w}}$ and $\varepsilon^{-1} b(\rho) \sqrt{\rho} \varphi(\rho) \Lambda^{s} \hat{\mathbf{w}}$ are 'almost' potential, that is, equal to a gradient up to a remainder term bounded in $L^{2}$ by $C(r, s, d) \eta\|(\nabla \hat{\mathbf{z}}, \nabla \hat{\rho})\|_{L^{\infty}}\|(\hat{\rho}, \hat{\mathbf{z}})\|_{H^{s}}$ (like $\mathbf{R}$ ), we see that $\mathcal{I}$ reduces to

$$
\mathcal{I}=-\frac{1}{\varepsilon} \int_{\mathbb{R}^{d}}\left\langle\hat{\mathbf{Y}}^{s}, b(\rho) \sqrt{\rho} \psi(\rho) \Lambda^{s} \hat{\mathbf{w}}\right\rangle d X+\mathcal{R}=-\frac{1}{\varepsilon} \int_{\mathbb{R}^{d}} b(\rho) \rho \psi^{2}(\rho) \Lambda^{s} \mathbf{u} \cdot \Lambda^{s} \hat{\mathbf{w}} d X+\mathcal{R}
$$

with $|\mathcal{R}| \leqslant C(r, s, d) \eta\|(\nabla \hat{\mathbf{z}}, \nabla \hat{\rho})\|_{L^{\infty}}\|(\hat{\rho}, \hat{\mathbf{z}})\|_{H^{s}}^{2}$. Similarly as what is done in the potential case (in the previous section), this remaining $\mathcal{O}\left(\varepsilon^{-1}\right)$ in $\mathcal{I}$ can be cancelled out by adding to $\frac{d}{d T} \frac{1}{2}\left(\left\|\mathcal{Q} \hat{\mathbf{Y}}^{s}\right\|_{L^{2}}^{2}+\left\|\mathcal{P} \hat{\mathbf{X}}^{s}\right\|_{L^{2}}^{2}\right)$ the time derivative

$$
\begin{array}{r}
\frac{d}{d T} \int_{\mathbb{R}^{d}} \frac{1}{2} \psi^{2}(\rho) g^{\prime}(\rho)\left(\Lambda^{s} \hat{\rho}\right)^{2} d X=-\eta \int_{\mathbb{R}^{d}}\left(\hat{\mathbf{u}} \cdot \nabla\left(\psi^{2}(\rho) g^{\prime}(\rho)\right)+\rho \partial_{\rho}\left(\psi^{2}(\rho) g^{\prime}(\rho)\right) \nabla \cdot \hat{\mathbf{u}}\right)\left(\Lambda^{s} \hat{\rho}\right)^{2} d X \\
\left.+\eta \int_{\mathbb{R}^{d}} \nabla \cdot\left(\psi^{2}(\rho) g^{\prime}(\rho)\right) \hat{\mathbf{u}}\right)\left(\Lambda^{s} \hat{\rho}\right)^{2} d X-\int_{\mathbb{R}^{d}} \rho \psi^{2}(\rho) g^{\prime}(\rho)\left(\Lambda^{s} \hat{\rho}\right) \nabla \cdot \Lambda^{s} \hat{\mathbf{u}} d X= \\
\mathcal{R}_{1}+\int_{\mathbb{R}^{d}} \rho \psi^{2}(\rho) g^{\prime}(\rho) \nabla\left(\Lambda^{s} \hat{\rho}\right) \cdot \Lambda^{s} \hat{\mathbf{u}} d X
\end{array}
$$


with $\left|\mathcal{R}_{1}\right| \leqslant C(r, s, d) \eta\|(\nabla \hat{\mathbf{z}}, \nabla \hat{\rho})\|_{L^{\infty}}\|(\hat{\rho}, \hat{\mathbf{z}})\|_{H^{s}}^{2}$. Now, observing that

$$
\nabla\left(\Lambda^{s} \hat{\rho}\right)=\frac{1}{\varepsilon} \Lambda^{s}\left(\frac{\rho \hat{\mathbf{w}}}{a(\rho)}\right)=\frac{\rho}{\varepsilon a(\rho)} \Lambda^{s} \hat{\mathbf{w}}+\mathbf{R}_{1}
$$

with $\left\|\mathbf{R}_{1}\right\|_{L^{2}} \leqslant C(r, s, d) \eta\|(\nabla \hat{\mathbf{z}}, \nabla \hat{\rho})\|_{L^{\infty}}\|(\hat{\rho}, \hat{\mathbf{z}})\|_{H^{s}}$ (like $\mathbf{R}$ again), and recalling that $b(\rho)=$ $\frac{\rho g^{\prime}(\rho)}{a(\rho)}$, we arrive at

$$
\left|\mathcal{I}+\frac{d}{d T} \int_{\mathbb{R}^{d}} \frac{1}{2} \psi^{2}(\rho) g^{\prime}(\rho)\left(\Lambda^{s} \hat{\rho}\right)^{2} d X\right| \leqslant C(r, s, d) \eta\|(\nabla \hat{\mathbf{z}}, \nabla \hat{\rho})\|_{L^{\infty}}\|(\hat{\rho}, \hat{\mathbf{z}})\|_{H^{s}}^{2} .
$$

Appropriate choices of $\psi$ and $\varphi$ will enable us to get rid of the other tricky terms, exactly as in [4]. Of course, there is no reason to change $\psi$, and we set $\psi^{2}(\rho)=a^{s}(\rho)$ as in the potential case. As regards $\varphi$, it turns out that a good choice is given by

$$
\varphi^{2}(\rho)=\frac{A(\rho)}{\rho}, \text { with } A^{\prime}(\rho)=a^{s}(\rho)-\rho \partial_{\rho}\left(a^{s}(\rho)\right) .
$$

For convenience, we keep abstract notations for $\psi$ and $\varphi$ in what follows, and use only that $\psi^{2}(\rho)=a^{s}(\rho)$ to simplify the equation satisfied by $\hat{\mathbf{Y}}^{s}$. Since $\nabla \cdot \mathcal{P} \equiv 0$, we have

$$
\begin{gathered}
\int_{\mathbb{R}^{d}}\left\langle\mathcal{Q} \hat{\mathbf{Y}}^{s}, i \nabla\left(a(\rho) \nabla \cdot \hat{\mathbf{Y}}^{s}\right)\right\rangle=\int_{\mathbb{R}^{d}}\left\langle\hat{\mathbf{Y}}^{s}, i \nabla\left(a(\rho) \nabla \cdot \hat{\mathbf{Y}}^{s}\right)\right\rangle=0, \\
\int_{\mathbb{R}^{d}}\left\langle\mathcal{P} \hat{\mathbf{X}}^{s}, i \nabla\left(a(\rho) \nabla \cdot \hat{\mathbf{X}}^{s}\right)\right\rangle=0 .
\end{gathered}
$$

From our previous computations this implies

$$
\begin{array}{r}
\frac{d}{d T} \frac{1}{2}\left(\left\|\mathcal{Q} \hat{\mathbf{Y}}^{s}\right\|_{L^{2}}^{2}+\left\|\mathcal{P} \hat{\mathbf{X}}^{s}\right\|_{L^{2}}^{2}+\int_{\mathbb{R}^{d}} \psi^{2}(\rho) g^{\prime}(\rho)\left(\Lambda^{s} \hat{\rho}\right)^{2} d X\right)=\mathcal{R}_{2} \\
+\int_{\mathbb{R}^{d}}\left\langle\mathcal{Q} \hat{\mathbf{Y}}^{s},-i \varepsilon a(\rho)\left(\nabla_{0} \hat{\mathbf{Y}}^{s}\right) \nabla \log (\sqrt{\rho} \psi(\rho))+i \varepsilon s \sqrt{\rho} \psi(\rho)\left(\nabla \mathcal{P} \Lambda^{s} \hat{\mathbf{z}}\right) \nabla a(\rho)\right\rangle d X \\
+\int_{\mathbb{R}^{d}}\left\langle\mathcal{P} \hat{\mathbf{X}}^{s},-i \varepsilon a(\rho)\left(\nabla_{0} \hat{\mathbf{X}}^{s}\right) \nabla \log \left(\frac{\sqrt{\rho} a^{s}(\rho)}{\varphi(\rho)}\right)+i \varepsilon s \sqrt{\rho} \varphi(\rho)\left(\nabla \mathcal{P} \Lambda^{s} \hat{\mathbf{z}}\right) \nabla a(\rho)\right\rangle d X \\
-\int_{\mathbb{R}^{d}}\left\langle\mathcal{P} \hat{\mathbf{X}}^{s}, i \varepsilon a(\rho)\left(\nabla \cdot \hat{\mathbf{X}}^{s}\right) \nabla \log \left(\frac{a^{s}(\rho)}{\varphi^{2}(\rho)}\right)\right\rangle d X
\end{array}
$$

with $\left|\mathcal{R}_{2}\right| \leqslant C(r, s, d) \eta\|(\nabla \hat{\mathbf{z}}, \nabla \hat{\rho})\|_{L^{\infty}}\|(\hat{\rho}, \hat{\mathbf{z}})\|_{H^{s}}^{2}$. Let us concentrate for a while on the second line in (13) here above. By the same computations as in [4, pp.1516-1517], which heavily use that $\psi^{2}(\rho)=a^{s}(\rho)$ and rely on successive integrations by parts together with commutator estimates, it is found to be equal to

$$
\mathcal{R}_{3}+\frac{\varepsilon}{2} \int_{\mathbb{R}^{d}} \rho a^{s+1}(\rho)\left\langle\mathcal{Q} \Lambda^{s} \hat{\mathbf{z}}, i\left(\nabla \mathcal{P} \Lambda^{s} \hat{\mathbf{z}}\right) \nabla \log \left(\frac{a^{s}(\rho)}{\rho}\right)\right\rangle d X
$$


with $\left|\mathcal{R}_{3}\right| \leqslant C(r, s, d) \eta \varepsilon\left\|D^{2} \hat{\rho}\right\|_{L^{\infty}}\|\hat{\mathbf{z}}\|_{H^{s}}^{2}$. Furthermore, by a similar approach - as in [4, p.1518]-, the last two lines in (13) can be written as

$$
\mathcal{R}_{4}-\frac{\varepsilon}{2} \int_{\mathbb{R}^{d}} \rho a(\rho) \varphi^{2}(\rho)\left\langle\mathcal{Q} \Lambda^{s} \hat{\mathbf{z}}, i\left(\nabla \mathcal{P} \Lambda^{s} \hat{\mathbf{z}}\right) \nabla \log \left(\rho \varphi^{2}(\rho)\right)\right\rangle d X
$$

with $\left|\mathcal{R}_{4}\right| \leqslant C(r, s, d) \eta \varepsilon\left\|D^{2} \hat{\rho}\right\|_{L^{\infty}}\|\hat{\mathbf{z}}\|_{H^{s}}^{2}$. Therefore, the appropriate choice of $\varphi$ is dictated by the fact that we want to get rid of the terms involving $s+1$ derivatives of $\hat{\mathbf{z}}$. If we set $\varphi$ so that

$$
a^{s}(\rho) \nabla \log \left(\frac{a^{s}(\rho)}{\rho}\right)-\varphi^{2}(\rho) \nabla \log \left(\rho \varphi^{2}(\rho)\right)=0
$$

which is merely equivalent to

$$
\nabla\left(\rho \varphi^{2}(\rho)\right)=\left(a^{s}(\rho)-\rho \partial_{\rho}\left(a^{s}(\rho)\right)\right) \nabla \rho
$$

we deduce from 13 that

$$
\frac{d}{d T} \frac{1}{2}\left(\left\|\mathcal{Q} \hat{\mathbf{Y}}^{s}\right\|_{L^{2}}^{2}+\left\|\mathcal{P} \hat{\mathbf{X}}^{s}\right\|_{L^{2}}^{2}+\int_{\mathbb{R}^{d}} \psi^{2}(\rho) g^{\prime}(\rho)\left(\Lambda^{s} \hat{\rho}\right)^{2} d X\right)=\mathcal{R}_{2}+\mathcal{R}_{3}+\mathcal{R}_{4} .
$$

On the one hand, the right-hand side has been estimated above by

$$
\mathcal{R}_{2}+\mathcal{R}_{3}+\mathcal{R}_{4} \leqslant C(r, s, d) \eta\left(\|(\nabla \hat{\mathbf{z}}, \nabla \hat{\rho})\|_{L^{\infty}}\|(\hat{\rho}, \hat{\mathbf{z}})\|_{H^{s}}^{2}+\varepsilon\left\|D^{2} \hat{\rho}\right\|_{L^{\infty}}\|\hat{\mathbf{z}}\|_{H^{s}}^{2}\right) .
$$

On the other hand, the expression

$$
\left\|\mathcal{Q} \hat{\mathbf{Y}}^{s}\right\|_{L^{2}}^{2}+\left\|\mathcal{P} \hat{\mathbf{X}}^{s}\right\|_{L^{2}}^{2}+\int_{\mathbb{R}^{d}} \psi^{2}(\rho) g^{\prime}(\rho)\left(\Lambda^{s} \hat{\rho}\right)^{2} d X
$$

is equivalent to $\left(\|\hat{\mathbf{u}}\|_{H^{s}}^{2}+\|\hat{\rho}\|_{H^{s}}^{2}+\varepsilon^{2}\|\hat{\rho}\|_{H^{s+1}}^{2}\right)$ as long as $\|\hat{\rho}\|_{W^{1, \infty}}$ remains bounded, as shown in Proposition 5 below. In addition, $\|\hat{\rho}\|_{W^{1, \infty}}$ is controlled by the Sobolev embedding $H^{s} \hookrightarrow$ $W^{1, \infty}$, which is valid since $s>1+d / 2$. We can thus complete the proof of Theorem 2 by a standard, Gronwall-type / bootstrap argument.

Proposition 5 Let $s$ be a positive real number. Let $r \in(0, \varrho / 2]$ be such that $g^{\prime}(\rho)>0$ and $K(\rho)>0$ if $|\rho-\varrho| \leqslant r$. We denote by $\psi$ and $\varphi$ the positive functions defined for $|\rho-\varrho| \leqslant r$ by

$$
a^{2}(\rho)=\rho K(\rho), \quad \psi^{2}(\rho)=a^{s}(\rho), \quad \rho \varphi^{2}(\rho)=2 \int_{\varrho-r}^{\rho} a^{s}+2 \max _{|\theta-\varrho| \leqslant r}\left(\theta a^{s}(\theta)\right)-\rho a^{s}(\rho),
$$

by $\Lambda^{s}$ the operator $(1-\Delta)^{s / 2}$, by $\mathcal{Q}$ the $L^{2}$-orthogonal projector onto potential vector fields, and by $\mathcal{P}=I-\mathcal{Q}$ the $L^{2}$-orthogonal projector onto solenoidal vector fields. Then for all $(\hat{\rho}, \hat{\mathbf{u}}) \in H^{s+1}\left(\mathbb{R}^{d}\right) \times\left(H^{s}\left(\mathbb{R}^{d}\right)\right)^{d}$ such that $\|\hat{\rho}\|_{W^{1, \infty}} \leqslant r$, for all $\eta \in(0,1]$, for all $\varepsilon>0$,

$$
\begin{aligned}
& c\left(\|\hat{\mathbf{u}}\|_{H^{s}}^{2}+\|\hat{\rho}\|_{H^{s}}^{2}+\varepsilon^{2}\|\hat{\rho}\|_{H^{s+1}}^{2}\right) \leqslant \\
& \left\|\mathcal{Q}\left(\sqrt{\rho} \psi(\rho) \Lambda^{s} \hat{\mathbf{z}}\right)\right\|_{L^{2}}^{2}+\left\|\mathcal{P}\left(\sqrt{\rho} \varphi(\rho) \Lambda^{s} \hat{\mathbf{z}}\right)\right\|_{L^{2}}^{2}+\left\|\sqrt{g^{\prime}(\rho)} \psi(\rho) \Lambda^{s} \hat{\rho}\right\|_{L^{2}}^{2} \leqslant \\
& C\left(\|\hat{\mathbf{u}}\|_{H^{s}}^{2}+\|\hat{\rho}\|_{H^{s}}^{2}+\varepsilon^{2}\|\hat{\rho}\|_{H^{s+1}}^{2}\right),
\end{aligned}
$$

where $\rho=\varrho+\eta \hat{\rho}, \hat{\mathbf{z}}=\hat{\mathbf{u}}+i \varepsilon \frac{a(\rho)}{\rho} \nabla \hat{\rho}$, and the constants $c>0$ and $C>0$ depend only on $r$, $s, d$ (and the functions $g, K$ ). 
The proof is to some extent similar to that of Proposition 3. We just have to check that $\left\|\mathcal{Q}\left(\sqrt{\rho} \psi(\rho) \Lambda^{s} \hat{\mathbf{z}}\right)\right\|_{L^{2}}^{2}+\left\|\mathcal{P}\left(\sqrt{\rho} \varphi(\rho) \Lambda^{s} \hat{\mathbf{z}}\right)\right\|_{L^{2}}^{2}$ is 'equivalent' to $\|\hat{\mathbf{z}}\|_{H^{s}}^{2}$, i.e. there exist $c>0$ and $C>0$ depending only on $r, s, d$ such that

$$
c\|\hat{\mathbf{z}}\|_{H^{s}}^{2} \leqslant\left\|\mathcal{Q}\left(\sqrt{\rho} \psi(\rho) \Lambda^{s} \hat{\mathbf{z}}\right)\right\|_{L^{2}}^{2}+\left\|\mathcal{P}\left(\sqrt{\rho} \varphi(\rho) \Lambda^{s} \hat{\mathbf{z}}\right)\right\|_{L^{2}}^{2} \leqslant C\|\hat{\mathbf{z}}\|_{H^{s}}^{2} .
$$

The inequality on the right is straightforward since $\mathcal{Q}$ and $\mathcal{P}$ are projectors in $L^{2}$, and $\rho$, $\psi(\rho)$ and $\varphi(\rho)$ are bounded for $|\rho-\varrho| \leqslant r$, which is the case for $\rho=\varrho+\eta \hat{\rho}$ as soon as $\|\hat{\rho}\|_{L^{\infty}} \leqslant r$ and $\eta \in(0,1]$. Furthermore, since $\rho, \psi(\rho)$ and $\varphi(\rho)$ are positive and bounded away from zero for $|\rho-\varrho| \leqslant r$, there exists $C(r)$ such that

$\|\hat{\mathbf{z}}\|_{H^{s}}=\left\|\Lambda^{s} \hat{\mathbf{z}}\right\|_{L^{2}} \leqslant\left\|\mathcal{Q} \Lambda^{s} \hat{\mathbf{z}}\right\|_{L^{2}}+\left\|\mathcal{P} \Lambda^{s} \hat{\mathbf{z}}\right\|_{L^{2}} \leqslant C(r)\left(\left\|\sqrt{\rho} \psi(\rho) \mathcal{Q} \Lambda^{s} \hat{\mathbf{z}}\right\|_{L^{2}}+\left\|\sqrt{\rho} \varphi(\rho) \mathcal{P} \Lambda^{s} \hat{\mathbf{z}}\right\|_{L^{2}}\right)$.

Finally,

$$
\left\|\sqrt{\rho} \varphi(\rho) \mathcal{P} \Lambda^{s} \hat{\mathbf{z}}\right\|_{L^{2}} \leqslant\left\|\mathcal{P}\left(\sqrt{\rho} \varphi(\rho) \Lambda^{s} \hat{\mathbf{z}}\right)\right\|_{L^{2}}+\left\|[\sqrt{\rho} \varphi(\rho), \mathcal{P}] \Lambda^{s} \hat{\mathbf{z}}\right\|_{L^{2}}
$$

and the commutator here above enjoys an estimate of the form

$$
\left\|[\sqrt{\rho} \varphi(\rho), \mathcal{P}] \Lambda^{s} \hat{\mathbf{z}}\right\|_{L^{2}} \leqslant C(r, s, d)\|\hat{\mathbf{z}}\|_{H^{s-1}} .
$$

(See Lemma A.4 in [4. This estimate has in fact already been used, in a hidden way, in the estimate of $\mathcal{R}_{4}$ above.) We can proceed in a similar manner for $\left\|\mathcal{Q} \Lambda^{s} \hat{\mathbf{z}}\right\|_{L^{2}}$, and thus prove the inequality on the left in (14).

\section{Free wave regime}

Theorem 3 We choose a real number $s$ with $s>1+\frac{d}{2}$, and a positive real number $M$. For $\eta>0, \varepsilon>0$, any initial data

$\left(\hat{\rho}^{\text {in }}, \hat{\mathbf{u}}^{\text {in }}\right) \in \mathcal{B}_{s, \varepsilon}(M)=\left\{(\hat{\rho}, \hat{\mathbf{u}}) \in H^{s+1}\left(\mathbb{R}^{d}\right) \times\left(H^{s}\left(\mathbb{R}^{d}\right)\right)^{d} ;\|(\hat{\rho}, \hat{\mathbf{u}})\|_{\left.\left(H^{s}\left(\mathbb{R}^{d}\right)\right)^{d+1}+\varepsilon\|\hat{\rho}\|_{H^{s+1}\left(\mathbb{R}^{d}\right)} \leqslant M\right\}} \leqslant\right.$

is associated with the solution $(\hat{\rho}, \hat{\mathbf{u}}) \in \mathscr{C}\left(\left[0, T_{*} / \eta\right], \mathcal{B}_{s, \varepsilon}(2 M)\right)$ of $\left(\mathrm{EK}_{\varepsilon, \eta}\right)$ given by Theorem 2. Let $(\mathfrak{r}, \mathfrak{u}) \in \mathscr{C}_{b}\left(\mathbb{R}_{+}, H^{s}\left(\mathbb{R}^{d}\right) \times\left(H^{s}\left(\mathbb{R}^{d}\right)\right)^{d}\right)$ solve the acoustic equations

$$
\left\{\begin{array}{l}
\partial_{T} \mathfrak{r}+\varrho \nabla_{X} \cdot \mathfrak{u}=0 \\
\partial_{T} \mathfrak{u}+g^{\prime}(\varrho) \nabla_{X} \mathfrak{r}=0,
\end{array}\right.
$$

with initial data $(\mathfrak{r}, \mathfrak{u})_{\mid T=0}=\left(\hat{\rho}^{\mathrm{in}}, \hat{\mathbf{u}}^{\mathrm{in}}\right)$. Then at each time $T \in\left[0, T_{*} / \eta\right]$ we have

$$
\|(\hat{\rho}, \hat{\mathbf{u}})-(\mathfrak{r}, \mathfrak{u})\|_{H^{s-2}\left(\mathbb{R}^{d}\right) \times\left(H^{s-2}\left(\mathbb{R}^{d}\right)\right)^{d}} \leqslant C(\eta+\varepsilon) T,
$$

and

$$
\|(\hat{\rho}, \hat{\mathbf{u}})-(\mathfrak{r}, \mathfrak{u})\|_{H^{s-3}\left(\mathbb{R}^{d}\right) \times\left(H^{s-3}\left(\mathbb{R}^{d}\right)\right)^{d}} \leqslant C\left(\eta+\varepsilon^{2}\right) T,
$$

where $C$ depends only on $M, s$ and $d$. 
Remark 2 For solutions $(\mathfrak{r}, \mathfrak{u})$ of $(\mathrm{W})$ it is clear that the divergence-free part of the velocity field $\mathfrak{u}$ remains constant in time.

The difference between the estimates in $(15)$ and (16) is the regularity index. When $\varepsilon \lesssim \eta$, both estimates (15) and (16) provide an $\mathcal{O}(\eta T)$ error. By contrast, when $\varepsilon^{2} \lesssim \eta \ll \varepsilon$, they yield respectively an $\mathcal{O}(\varepsilon T)$ and an $\mathcal{O}(\eta T)$ error, so that the second one is smaller. Therefore, $\eta \approx \varepsilon$ appears to be a threshold at which we lose one derivative. The estimates (15) and (16) provide an $L^{\infty}$ error bound only for $s>2+d / 2$ and $s>3+d / 2$ respectively.

In the special case corresponding to the Gross-Pitaevskii equation $(K(\rho)=1 /(4 \rho)$, $g(\varrho)=\varrho-1$ ), the first rigorous justification of the free wave regime was given by Colin and Soyeur [16] in a bounded domain in terms of weak convergence. Strong convergence was proved much more recently by Béthuel, Danchin and Smets [6].

Proof of Theorem 3. By $\left(\mathrm{EK}_{\varepsilon, \eta}\right)$ and $(\mathrm{W})$, we see that $(\hat{\rho}-\mathfrak{r}, \hat{\mathbf{u}}-\mathfrak{u})$ solves

$$
\left\{\begin{aligned}
\partial_{T}(\hat{\rho}-\mathfrak{r})+\varrho \nabla_{X} \cdot(\hat{\mathbf{u}}-\mathfrak{u})= & -\eta \nabla_{X} \cdot(\hat{\rho} \hat{\mathbf{u}}) \\
\partial_{T}(\hat{\mathbf{u}}-\mathfrak{r})+g^{\prime}(\varrho) \nabla_{X}(\hat{\rho}-\mathfrak{r})= & -\eta \hat{\mathbf{u}} \cdot \nabla_{X} \hat{\mathbf{u}}-\left[g^{\prime}(\varrho+\eta \hat{\rho})-g^{\prime}(\varrho)\right] \nabla_{X} \hat{\rho} \\
& +\varepsilon^{2} \nabla_{X}\left(K(\varrho+\eta \hat{\rho}) \Delta_{X} \hat{\rho}+\frac{\eta}{2} K^{\prime}(\varrho+\eta \hat{\rho})\left|\nabla_{X} \hat{\rho}\right|^{2}\right)
\end{aligned}\right.
$$

with null initial data. As in [6], the proof of Theorem 3 amounts to estimating the source terms in this system. By Theorem 2, we have

$$
\begin{gathered}
\left\|\eta \nabla_{X} \cdot(\hat{\rho} \hat{\mathbf{u}})\right\|_{H^{s-1}} \\
\leqslant C(s, d, M) \eta, \\
\left\|-\eta \hat{\mathbf{u}} \cdot \nabla_{X} \hat{\mathbf{u}}-\left[g^{\prime}(\varrho+\eta \hat{\rho})-g^{\prime}(\varrho)\right] \nabla_{X} \hat{\rho}+\varepsilon^{2} \nabla_{X}\left(K(\varrho+\eta \hat{\rho}) \Delta_{X} \hat{\rho}+\frac{\eta}{2} K^{\prime}(\varrho+\eta \hat{\rho})\left|\nabla_{X} \hat{\rho}\right|^{2}\right)\right\|_{H^{s-2}} \\
\leqslant C(s, d, M)\left(\eta+\varepsilon+\varepsilon^{2} \eta\right) \leqslant C(s, d, M)(\eta+\varepsilon),
\end{gathered}
$$

where we have used the bound on $\varepsilon \hat{\rho}$ in $H^{s+1}$ for the term $\varepsilon^{2} \nabla_{X} \Delta_{X} \hat{\rho}$, and

$$
\begin{array}{r}
\left\|-\eta \hat{\mathbf{u}} \cdot \nabla_{X} \hat{\mathbf{u}}-\left[g^{\prime}(\varrho+\eta \hat{\rho})-g^{\prime}(\varrho)\right] \nabla_{X} \hat{\rho}+\varepsilon^{2} \nabla_{X}\left(K(\varrho+\eta \hat{\rho}) \Delta_{X} \hat{\rho}+\frac{\eta}{2} K^{\prime}(\varrho+\eta \hat{\rho})\left|\nabla_{X} \hat{\rho}\right|^{2}\right)\right\|_{H^{s-3}} \\
\leqslant C(s, d, M)\left(\eta+\varepsilon^{2}+\varepsilon^{2} \eta\right) \leqslant C(s, d, M)\left(\eta+\varepsilon^{2}\right),
\end{array}
$$

using this time that $\varepsilon^{2} \nabla_{X} \Delta_{X} \hat{\rho}$ in $H^{s-3}$ is bounded by $\varepsilon^{2}\|\hat{\rho}\|_{H^{s}}$. The conclusion follows from Duhamel's formula and the fact that the wave group is unitary on $H^{s}$.

In one space dimension $(d=1)$, solutions to the acoustic equations in (W) are exactly combinations of left-going and right-going waves. More precisely, there exist $\mathrm{w}$ and $\mathrm{v}$ such that

$$
\frac{1}{2}\left(\mathfrak{r}+\frac{\varrho}{\mathfrak{c}} \mathfrak{u}\right)(T, X)=\mathrm{w}(X-\mathfrak{c} T) \quad \text { and } \quad \frac{1}{2}\left(\mathfrak{r}-\frac{\varrho}{\mathfrak{c}} \mathfrak{u}\right)(T, X)=\mathrm{v}(X+\mathfrak{c} T) .
$$

In what follows, we aim at characterizing the counterpart of these linear waves at the weakly nonlinear, and possibly weakly dispersive level. 


\section{One-way propagating waves}

In this section, the space dimension is $d=1$, and the fluid velocity is denoted by $u$ instead of the bold letter $\mathbf{u}$. We are going to show that the evolution of the two weakly nonlinear / weakly dispersive counter propagating waves is governed by Burgers equations if the parameters $\eta$ and $\varepsilon$ are of the same order, by weakly dispersive Korteweg-de Vries (KdV) equations if $\varepsilon^{2} \ll \eta$, and by regular $\mathrm{KdV}$ equations if $\eta$ and $\varepsilon^{2}$ are of the same order. What remains of the reference density $\varrho$ in these equations lies in the two quantities pointed out in the introduction and defined by

$$
\Gamma \stackrel{\text { def }}{=} \frac{3 \mathfrak{c}}{2 \varrho}+\frac{\varrho g^{\prime \prime}(\varrho)}{2 \mathfrak{c}}, \quad \kappa \stackrel{\text { def }}{=} \frac{\varrho}{2 \mathfrak{c}} K(\varrho),
$$

which already appeared in a special form in earlier results on (NLS) by the first author [15, 14, 12, 11].

\subsection{Statement of errors bounds in various asymptotic regimes}

A first, simpler result holds when the left-going wave $\mathrm{v}$ is negligible, so that $\mathfrak{c} \hat{\rho} \approx \varrho \hat{u}$ (at least for small enough $T$ ) by (15) and (16). More precisely, we are going to show that, if the initial norm of the difference $\hat{\rho}-\varrho \hat{u} / \mathfrak{c}$ is small enough, then both $\hat{\rho}$ and $\varrho \hat{u} / \mathfrak{c}$ are either close to solutions $\mathfrak{Z}$ to the (inviscid) Burgers equation

$$
\partial_{\theta} \mathfrak{Z}+\Gamma \mathfrak{Z} \partial_{X} \mathfrak{Z}=0
$$

if $\varepsilon \lesssim \eta$ or $\varepsilon^{2} \ll \eta$, or close to solutions $\zeta$ to the $\mathrm{KdV}$ equation

$$
\partial_{\theta} \zeta+\Gamma \zeta \partial_{X} \zeta=\frac{\varepsilon^{2}}{\eta} \kappa \partial_{X}^{3} \zeta
$$

if $\varepsilon^{2}=\mathcal{O}(\eta)$. Note that this equation is clearly weakly dispersive if $\varepsilon^{2} \ll \eta$, and reduces to

$$
\partial_{\theta} \zeta+\Gamma \zeta \partial_{X} \zeta=\kappa \partial_{X}^{3} \zeta
$$

when $\eta=\varepsilon^{2}$. Precise error bounds are given in the following.

Theorem 4 We assume $d=1$, and take two real numbers $s \geqslant 4$ and $M>0$. For $\eta \in(0,1]$, $\varepsilon \in(0,1]$, any initial data

$$
\left(\hat{\rho}^{\text {in }}, \hat{u}^{\text {in }}\right) \in \mathcal{B}_{s, \varepsilon}(M)=\left\{(\hat{\rho}, \hat{u}) \in H^{s+1}(\mathbb{R}) \times H^{s}(\mathbb{R}) ;\|(\hat{\rho}, \hat{u})\|_{\left(H^{s}(\mathbb{R})\right)^{2}}+\varepsilon\|\hat{\rho}\|_{H^{s+1}(\mathbb{R})} \leqslant M\right\}
$$

is associated with the solution $(\hat{\rho}, \hat{u}) \in \mathscr{C}\left(\left[0, T_{*} / \eta\right], \mathcal{B}_{s, \varepsilon}(2 M)\right)$ of $\left(\mathrm{EK}_{\varepsilon, \eta}\right)$ such that $(\rho, u)(0)=$ $\left(\rho^{\mathrm{in}}, u^{\mathrm{in}}\right)$, as given by Theorem 2 in the case $d=1$. We also introduce $\mathfrak{Z} \in \mathscr{C}\left(\left[0, \theta_{*}\right], H^{s}(\mathbb{R})\right)$ a smooth solution of the inviscid Burgers equation

$$
\partial_{\theta} \mathfrak{Z}+\Gamma \mathfrak{Z} \partial_{X} \mathfrak{Z}=0
$$


such that $\mathfrak{Z}(0)=\hat{\rho}^{\text {in }}$, where the time of existence $\theta_{*}$ depends continuously on $M$, and $\zeta \in$ $\mathscr{C}\left([0,+\infty), H^{s}(\mathbb{R})\right)$ the global solution of the $\mathrm{KdV}$ equation

$$
\partial_{\theta} \zeta+\Gamma \zeta \partial_{X} \zeta=\frac{\varepsilon^{2}}{\eta} \kappa \partial_{X}^{3} \zeta
$$

such that $\zeta(0)=\hat{\rho}^{\text {in }}$. Then there exists a constant $C$, depending only on $s$ and $M$, so that for $0 \leqslant T \leqslant \min \left(T_{*}, \theta_{*}\right) / \eta$, the following hold:

(i). If $\sigma \in[0, s-3]$, or if $(s>7 / 2$ and $\sigma \in[0, s-2])$,

$$
\begin{aligned}
\|\hat{\rho}-\mathfrak{Z}(\eta T,-\mathfrak{c} T)\|_{H^{\sigma}(\mathbb{R})}+ & \left\|\frac{\varrho}{\mathfrak{c}} \hat{u}-\mathfrak{Z}(\eta T, \cdot-\mathfrak{c} T)\right\|_{H^{\sigma}(\mathbb{R})} \\
& \leqslant C\left(\eta+\varepsilon+\frac{\varepsilon^{2}}{\eta}+\left\|\hat{\rho}^{\text {in }}-\frac{\varrho}{\mathfrak{c}} \hat{u}^{\text {in }}\right\|_{H^{\sigma+1}(\mathbb{R})}\right),
\end{aligned}
$$

If $(s \geqslant 4$ and $\sigma \in[0, s-4])$, or if $(s>9 / 2$ and $\sigma \in[0, s-3])$,

$$
\begin{aligned}
\|\hat{\rho}-\mathfrak{Z}(\eta T, \cdot-\mathfrak{c} T)\|_{H^{\sigma}(\mathbb{R})}+ & \left\|\frac{\varrho}{\mathfrak{c}} \hat{u}-\mathfrak{Z}(\eta T, \cdot-\mathfrak{c} T)\right\|_{H^{\sigma}(\mathbb{R})} \\
& \leqslant C\left(\eta+\frac{\varepsilon^{2}}{\eta}+\left\|\hat{\rho}^{\text {in }}-\frac{\varrho}{\mathfrak{c}} \hat{u}^{\text {in }}\right\|_{H^{\sigma+1}(\mathbb{R})}\right),
\end{aligned}
$$

(ii). For all $\sigma \in[0, s-4]$,

$$
\begin{aligned}
\|\hat{\rho}-\zeta(\eta T,-\mathfrak{c} T)\|_{H^{\sigma}(\mathbb{R})}+ & \left\|\frac{\varrho}{\mathfrak{c}} \hat{u}-\zeta(\eta T, \cdot-\mathfrak{c} T)\right\|_{H^{\sigma}(\mathbb{R})} \\
& \leqslant C\left(1+\frac{\varepsilon^{2}}{\eta}\right)\left(\eta+\varepsilon+\left\|\hat{\rho}^{\text {in }}-\frac{\varrho}{\mathfrak{c}} \hat{u}^{\text {in }}\right\|_{H^{\sigma+3}(\mathbb{R})}\right) .
\end{aligned}
$$

If, in addition $s \geqslant 5$, then for all $\sigma \in[0, s-5]$,

$$
\begin{aligned}
\|\hat{\rho}-\zeta(\eta T, \cdot-\mathfrak{c} T)\|_{H^{\sigma}(\mathbb{R})}+ & \left\|\frac{\varrho}{\mathfrak{c}}-\zeta(\eta T, \cdot-\mathfrak{c} T)\right\|_{H^{\sigma}(\mathbb{R})} \\
& \leqslant C\left(1+\frac{\varepsilon^{2}}{\eta}\right)\left(\eta+\varepsilon^{2}+\left\|\hat{\rho}^{\text {in }}-\frac{\varrho}{\mathfrak{c}} \hat{u}^{\text {in }}\right\|_{H^{\sigma+3}(\mathbb{R})}\right) .
\end{aligned}
$$

Observe that both $\mathfrak{Z}$ and $\zeta$ are shifted to the right at speed $\mathfrak{c}$ in the estimates above. This theorem provides various types of errors, depending on the relation between $\eta$ and $\varepsilon$. Roughly speaking and neglecting the term $\left\|\hat{\rho}^{\text {in }}-\varrho \hat{u}^{\text {in }} / \mathfrak{c}\right\|_{H^{\sigma}(\mathbb{R})}$, which will be small enough provided that the initial data are well-prepared, Theorem 4 ensures that $\hat{\rho} \approx \varrho \hat{u} / \mathfrak{c}$ is close, up to a rescaling in time and space shifting, to the solution to

- the Burgers equation (17) if $\varepsilon \lesssim \eta \ll 1$, with an $\mathcal{O}(\eta)$ error;

- still the Burgers equation (17) if $\varepsilon^{2} \ll \eta \ll \varepsilon \ll 1$, with an $\mathcal{O}\left(\varepsilon^{2} / \eta\right)$ error; 
- but also the $\mathrm{KdV}$ equation (18) if $\varepsilon^{2} \ll \eta \ll \varepsilon \ll 1$ (which makes (18) weakly dispersive), with a smaller error $\mathcal{O}(\eta)$ (because $\eta \ll \varepsilon^{2} / \eta$ ) if we use (ii),

- and the KdV equation (18) if $\varepsilon^{2} \approx \eta \ll 1$, with an $\mathcal{O}(\eta)$ error.

When $\eta \ll \varepsilon^{2}$, the comparison with the solution $\mathfrak{Z}$ of the Burgers equation given in the inequalities of $(i)$ are clearly meaningless. In the first statement of (i), we have in the error term $2 \varepsilon \leqslant \eta+\frac{\varepsilon^{2}}{\eta}$, so that the term $\varepsilon$ seems superfluous, but we have kept it in order to see the $\frac{\varepsilon^{2}}{\eta}$ gain in the second statement, which is the dispersive term $\left(\varepsilon^{2} / \eta\right) \kappa \partial_{X}^{3} \zeta$ of the $\mathrm{KdV}$ equation.

Note also that both statements (i) and (ii) hold true if $s \geqslant 5$. For instance in the case $\varepsilon \lesssim \eta \ll 1$, both $(i)$ and (ii) yield $\mathcal{O}(\eta)$ errors, but the advantage of the second statements are that they control one more derivative. However, this advantage is lost in the case $\varepsilon^{2} \ll \eta \ll \varepsilon \ll 1$, for which the first statement in (i) and (ii) provides $\mathcal{O}(\varepsilon)$ errors instead of the 'natural' $\mathcal{O}(\eta)$. In this sense, the case $\varepsilon \approx \eta$ corresponds to a threshold across which the natural, $\mathcal{O}(\eta)$ estimates lose the control of one derivative.

The coefficient $\varepsilon^{2} / \eta$ in the dispersive term in (18) may be removed using the scaling invariance of $(\mathrm{KdV})$. Indeed, if $\zeta$ solves (18), then the function

$$
\zeta_{\sharp}(\theta, X) \stackrel{\text { def }}{=} \frac{\eta}{\varepsilon^{2}} \zeta\left(\frac{\eta}{\varepsilon^{2}} \theta, X\right)
$$

solves

$$
\partial_{\theta} \zeta_{\sharp}+\Gamma \zeta_{\sharp} \partial_{X} \zeta_{\sharp}=\kappa \partial_{X}^{3} \zeta_{\sharp}
$$

with associated initial datum $\zeta_{\sharp}(\theta=0, X)=\left(\eta / \varepsilon^{2}\right) \zeta(0, X)$, which may be large or small depending on the ratio $\eta / \varepsilon^{2}$. In particular, $\left\|\zeta_{\sharp}(\theta=0)\right\|_{H^{s}}=\left(\eta / \varepsilon^{2}\right)\|\zeta(\theta=0)\|_{H^{s}}$ for any $s$.

Our results subsume those of [8] and [11] as particular cases. Notice that in Corollary 1 of [11], there are two misprints: the correct conclusion is $\sup _{\left[0, \min \left(\tau_{0}, \tau_{*}\right)\right]}\left\|A_{\varepsilon}-\zeta_{\varepsilon}\right\|_{H^{s-5}}+\| U_{\varepsilon}-$ $\zeta_{\varepsilon} \|_{H^{s-5}} \leqslant K\left(\left\|A_{\varepsilon}^{\text {in }}-U_{\varepsilon}^{\text {in }}\right\|_{H^{s-2}}+\varepsilon^{2}\right)$.

\subsection{Proof of error bounds for right-going waves}

Concentrating on right-going waves, we can work in a moving frame, and introduce

$$
\tilde{\rho}(T, Y) \stackrel{\text { def }}{=} \hat{\rho}(T, Y+\mathfrak{c} T) \quad \tilde{u}(T, Y) \stackrel{\text { def }}{=} \hat{u}(T, Y+\mathfrak{c} T) .
$$

This changes $\left(\mathrm{EK}_{\varepsilon, \eta}\right)$ into

$$
\left\{\begin{array}{l}
\partial_{T} \tilde{\rho}-\mathfrak{c} \partial_{Y} \tilde{\rho}+\partial_{Y}((\varrho+\eta \tilde{\rho}) \tilde{u})=0 \\
\partial_{T} \tilde{u}-\mathfrak{c} \partial_{Y} \tilde{u}+\eta \tilde{u} \partial_{Y} \tilde{u}+g^{\prime}(\varrho+\eta \tilde{\rho}) \partial_{Y} \tilde{\rho} \\
\quad=\varepsilon^{2} \partial_{Y}\left(K(\varrho+\eta \tilde{\rho}) \partial_{Y}^{2} \tilde{\rho}+\frac{\eta}{2} K^{\prime}(\varrho+\eta \tilde{\rho})\left(\partial_{Y} \tilde{\rho}\right)^{2}\right) .
\end{array}\right.
$$

In a first step, we estimate the $L^{2}$ norm of $\tilde{\rho}-\varrho c^{-1} \tilde{u}$. 
Lemma 1 We assume that $s \geqslant 3$. Then, in the framework of Theorem 4, there exists a constant $C$, depending only on $s$ and $M$, so that

$$
\sup _{0 \leqslant T \leqslant T_{*} / \eta}\left\|\tilde{\rho}-\frac{\varrho}{\mathfrak{c}} \tilde{u}\right\|_{L^{2}} \leqslant C\left\|\tilde{\rho}^{\text {in }}-\frac{\varrho}{\mathfrak{c}} \tilde{u}^{\text {in }}\right\|_{L^{2}}+C\left(\eta+\varepsilon^{2}\right) .
$$

Proof. We argue as in the proof of Proposition 14 in [8] (see also Proposition 2 in [11]). Throughout the following computations, $C$ will denote a positive constant depending only on $s$ and $M$ that may change from line to line. From (19), we see that the difference

$$
\mathrm{v} \stackrel{\text { def }}{=} \tilde{\rho}-\varrho c^{-1} \tilde{u}
$$

satisfies

$$
\partial_{T} \mathrm{v}-2 \mathfrak{c} \partial_{Y} \mathrm{v}-\eta \frac{\mathfrak{c}}{\varrho} \mathrm{v} \partial_{Y} \mathrm{v}=\partial_{Y} G
$$

where

$$
G \stackrel{\text { def }}{=} \frac{\varrho}{\eta \mathfrak{c}}\left(g(\varrho+\eta \tilde{\rho})-g(\varrho)-\eta g^{\prime}(\varrho) \tilde{\rho}\right)-\varepsilon^{2} \frac{\varrho}{\mathfrak{c}}\left(K(\varrho+\eta \tilde{\rho}) \partial_{Y}^{2} \tilde{\rho}+\frac{\eta}{2} K^{\prime}(\varrho+\eta \tilde{\rho})\left(\partial_{Y} \tilde{\rho}\right)^{2}\right)-\eta \frac{\mathfrak{c}}{\varrho} \frac{\tilde{\rho}^{2}}{2} .
$$

The terms $\mathrm{v} \partial_{Y} \mathrm{v}$ and $\partial_{Y} \mathrm{v}$ in $(20)$ do not contribute to the time evolution of $\|\mathrm{v}\|_{L^{2}}$, and more precisely, (20) implies that

$$
\frac{d}{d T} \int_{\mathbb{R}} \mathrm{v}^{2} d Y=2 \int_{\mathbb{R}} \mathrm{v} \partial_{Y} G d Y=-\frac{1}{\mathfrak{c}} \int_{\mathbb{R}}\left(2 \mathfrak{c} \partial_{Y} \mathrm{v}\right) G d Y .
$$

Using again (20), we can substitute $\partial_{T} \mathrm{v}-\eta \mathfrak{c} \varrho^{-1} \mathrm{v} \partial_{Y} \mathrm{v}-\partial_{Y} G$ for $2 \mathfrak{c} \partial_{Y} \mathrm{v}$ in the previous identity, which gives after integration in time,

$$
\|\mathrm{v}\|_{L^{2}}^{2}=\left\|\hat{\rho}^{\hat{i n}}-\frac{\varrho}{\mathfrak{c}} \hat{u}^{\mathrm{in}}\right\|_{L^{2}}^{2}+\frac{1}{\mathfrak{c}} \int_{0}^{T} \int_{\mathbb{R}}\left(-\partial_{\tau} \mathrm{v}+\eta \stackrel{\mathfrak{c}}{\varrho} \mathrm{v} \partial_{Y} \mathrm{v}+\partial_{Y} G\right) G d Y d \tau .
$$

Of course the integral $\int_{\mathbb{R}} G \partial_{Y} G d Y$ vanishes at all times. Moreover, since $s \geqslant 3$, we have $\left\|\partial_{Y} G\right\|_{L^{\infty}} \leqslant C\|G\|_{H^{s}}$ by Sobolev embedding, and $\|G\|_{H^{s}} \leqslant C\left(\eta+\varepsilon^{2}\right) \leqslant C$. Thus, by integration by parts,

$$
\int_{\mathbb{R}} \eta\left(\mathrm{v} \partial_{Y} \mathrm{v}\right) G d Y=-\frac{\eta}{2} \int_{\mathbb{R}} \mathrm{v}^{2} \partial_{Y} G d Y \leqslant C \eta\left\|\partial_{Y} G\right\|_{L^{\infty}}\|\mathrm{v}\|_{L^{2}}^{2} \leqslant C \eta\|\mathrm{v}\|_{L^{2}}^{2} .
$$

Therefore, integrating by parts in time the remaining integral in $(22)$, we deduce, for $0 \leqslant$ $T \leqslant T_{*} / \eta$

$$
\begin{aligned}
\|\mathrm{v}\|_{L^{2}}^{2} \leqslant & \left\|\hat{\rho}^{\text {in }}-\frac{\varrho}{\mathfrak{c}} \hat{u}^{\mathrm{in}}\right\|_{L^{2}}^{2}+\frac{1}{\mathfrak{c}} \int_{\mathbb{R}}[\mathrm{v}(0) G(0)-\mathrm{v}(T) G(T)] d Y \\
& +\frac{1}{\mathfrak{c}} \int_{0}^{T} \int_{\mathbb{R}} \mathrm{v} \partial_{\tau} G d Y d \tau+C \eta \int_{0}^{T}\|\mathrm{v}\|_{L^{2}}^{2} d \tau .
\end{aligned}
$$


Combining the $L^{2}$ bound (since $\left.s \geqslant 3\right)\|G\|_{L^{2}} \leqslant C\left(\eta+\varepsilon^{2}\right.$ ) with Young's inequality, we infer

$$
\begin{aligned}
\frac{1}{\mathfrak{c}} \int_{\mathbb{R}}[\mathrm{v}(0) G(0)-\mathrm{v}(T) G(T)] d Y & \leqslant C\left(\eta+\varepsilon^{2}\right)\left(\|\mathrm{v}(T)\|_{L^{2}}+\|\mathrm{v}(0)\|_{L^{2}}\right) \\
& \leqslant \frac{1}{4}\|\mathrm{v}\|_{L^{2}}^{2}+C\left(\eta+\varepsilon^{2}\right)^{2}+C\left\|\hat{\rho}^{\text {in }}-\frac{\varrho}{\mathfrak{c}} \hat{u}^{\mathrm{in}}\right\|_{L^{2}}^{2} .
\end{aligned}
$$

As a consequence, putting the term $\frac{1}{4}\|\mathrm{v}\|_{L^{2}}^{2}$ in the left-hand side,

$$
\frac{3}{4}\|\mathrm{v}\|_{L^{2}}^{2} \leqslant C\left\|\hat{\rho}^{\mathrm{in}}-\frac{\varrho}{\mathfrak{c}} \hat{u}^{\mathrm{in}}\right\|_{L^{2}}^{2}+C\left(\eta+\varepsilon^{2}\right)^{2}+\frac{1}{\mathfrak{c}} \int_{0}^{T} \int_{\mathbb{R}} \mathrm{v} \partial_{\tau} G d Y d \tau+C \eta \int_{0}^{T}\|\mathrm{v}\|_{L^{2}}^{2} d \tau .
$$

Now, notice that from the first equation in (19) we have $\partial_{\tau} \tilde{\rho}-\mathfrak{c} \partial_{Y} \mathrm{v}=-\eta \partial_{Y}(\tilde{\rho} \tilde{u})$ with $s \geqslant 3$, thus

$$
\left\|\partial_{\tau} \tilde{\rho}-\mathfrak{c} \partial_{Y} \mathrm{v}\right\|_{H^{2}} \leqslant C \eta
$$

and this implies

$$
\begin{aligned}
\partial_{\tau} G= & \frac{\varrho}{\mathfrak{c}}\left(g^{\prime}(\varrho+\eta \tilde{\rho})-g^{\prime}(\varrho)\right) \partial_{\tau} \tilde{\rho}-\eta \frac{\mathfrak{c}}{\varrho} \tilde{\rho} \partial_{\tau} \tilde{\rho} \\
& \quad-\varepsilon^{2} \frac{\varrho}{\mathfrak{c}}\left(K(\varrho+\eta \tilde{\rho}) \partial_{Y}^{2} \partial_{\tau} \tilde{\rho}+\eta K^{\prime}(\varrho+\eta \tilde{\rho}) \partial_{\tau} \tilde{\rho} \partial_{Y}^{2} \tilde{\rho}\right. \\
& \left.\quad+\eta K^{\prime}(\varrho+\eta \tilde{\rho}) \partial_{Y} \tilde{\rho} \partial_{Y} \partial_{\tau} \tilde{\rho}+\frac{\eta^{2}}{2} K^{\prime \prime}(\varrho+\eta \tilde{\rho})\left(\partial_{Y} \tilde{\rho}\right)^{2} \partial_{\tau} \tilde{\rho}\right) \\
& \quad \varrho\left(g^{\prime}(\varrho+\eta \tilde{\rho})-g^{\prime}(\varrho)\right) \partial_{Y} \mathrm{v}-\eta \frac{\mathfrak{c}^{2}}{\varrho} \tilde{\rho} \partial_{Y} \mathrm{v}-\varepsilon^{2} \varrho K(\varrho) \partial_{Y}^{3} \mathrm{v}+\mathcal{O}_{L^{\infty}\left(\left[0, T_{*} / \eta\right], L^{2}\right)}\left(\eta\left(\varepsilon^{2}+\eta\right)\right) .
\end{aligned}
$$

We then deduce, by another use of Young's inequality,

$$
\begin{aligned}
\frac{3}{4}\|\mathrm{v}\|_{L^{2}}^{2} \leqslant & C\left\|\hat{\rho}^{\text {in }}-\frac{\varrho}{\mathfrak{c}} \hat{u}^{\text {in }}\right\|_{L^{2}}^{2}+C\left(\eta+\varepsilon^{2}\right)^{2}+C \eta \int_{0}^{T}\|\mathrm{v}\|_{L^{2}}^{2} d \tau \\
& +\frac{1}{\mathfrak{c}} \int_{0}^{T} \int_{\mathbb{R}}\left(\varrho\left(g^{\prime}(\varrho+\eta \tilde{\rho})-g^{\prime}(\varrho)\right) \partial_{Y} \mathrm{v}-\eta \frac{\mathfrak{c}^{2}}{\varrho} \tilde{\rho} \partial_{Y} \mathrm{v}-\varepsilon^{2} \varrho K(\varrho) \partial_{Y}^{3} \mathrm{v}\right) \mathrm{v} d Y d \tau .
\end{aligned}
$$

An integration by parts gives

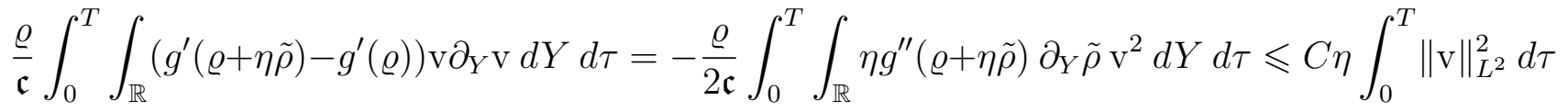
and similarly

$$
-\eta \int_{0}^{T} \int_{\mathbb{R}} \frac{\mathfrak{c}}{\varrho} \tilde{\rho} \partial_{Y} \mathrm{v} \mathrm{v} d Y d \tau=\frac{\eta \mathfrak{c}}{2 \varrho} \int_{0}^{T} \int_{\mathbb{R}} \mathrm{v}^{2} \partial_{Y} \tilde{\rho} d Y d \tau \leqslant C \eta \int_{0}^{T}\|\mathrm{v}\|_{L^{2}}^{2} d \tau .
$$

Furthermore, $\int_{\mathbb{R}} \mathrm{v} \partial_{Y}^{3} \mathrm{v} d Y=0$ for all times, hence

$$
\frac{3}{4}\|\mathrm{v}\|_{L^{2}}^{2} \leqslant C\left\|\hat{\rho}^{\text {in }}-\frac{\varrho}{\mathfrak{c}} \hat{u}^{\mathrm{in}}\right\|_{L^{2}}^{2}+C\left(\eta+\varepsilon^{2}\right)^{2}+C \eta \int_{0}^{T}\|\mathrm{v}\|_{L^{2}}^{2} d \tau .
$$


By Gronwall's lemma this implies

$$
\|\mathrm{v}\|_{L^{2}}^{2} \leqslant C\left(\left\|\hat{\rho}^{\text {in }}-\frac{\varrho}{\mathfrak{c}} \hat{u}^{\text {in }}\right\|_{L^{2}}^{2}+\left(\eta+\varepsilon^{2}\right)^{2}\right) \mathrm{e}^{C \eta T} .
$$

We can also estimate higher order, Sobolev norms of $\tilde{\rho}-\varrho c^{-1} \tilde{u}$. The natural idea is to differentiate the equation with respect to $Y$ and argue as for Lemma 1 (see Proposition 4 in [8], but here we lose three derivatives instead of six, which seems to be a misprint in [8]). However, we shall see that this does not yield an optimal result in terms of loss of derivatives (see Lemma 3 below).

Lemma 2 We assume that $s>7 / 2$. Then, in the framework of Theorem 4, for any $0 \leqslant$ $\sigma \leqslant s-3$, there exists $C$, depending only on $s, \sigma$ and $M$, so that

$$
\sup _{0 \leqslant T \leqslant T_{*} / \eta}\left\|\tilde{\rho}-\frac{\varrho}{\mathfrak{c}} \tilde{u}\right\|_{H^{\sigma}} \leqslant C\left(\left\|\hat{\rho}^{\text {in }}-\frac{\varrho}{\mathfrak{c}} \hat{u}^{\text {in }}\right\|_{H^{\sigma}}+\eta+\varepsilon^{2}\right) .
$$

Proof. We apply $\Lambda^{\sigma}$ to both sides in $(20)$ :

$$
\partial_{T} \Lambda^{\sigma} \mathrm{v}-2 \mathfrak{c} \partial_{Y} \Lambda^{\sigma} \mathrm{v}-\eta \frac{\mathfrak{c}}{\varrho} \Lambda^{\sigma}\left(\mathrm{v} \partial_{Y} \mathrm{v}\right)=\partial_{Y} \Lambda^{\sigma} G
$$

which implies

$$
\frac{d}{d T} \int_{\mathbb{R}}\left(\Lambda^{\sigma} \mathrm{v}\right)^{2} d Y=2 \eta \frac{\mathfrak{c}}{\varrho} \int_{\mathbb{R}} \Lambda^{\sigma} \mathrm{v} \Lambda^{\sigma}\left(\mathrm{v} \partial_{Y} \mathrm{v}\right) d Y+2 \int_{\mathbb{R}} \Lambda^{\sigma} \mathrm{v} \Lambda^{\sigma}\left(\partial_{Y} G\right) d Y
$$

Of course here the term ' $\mathrm{v} \partial_{Y} \mathrm{v}$ ' does have a contribution, which we can control in the usual way of dealing with Burgers' equation. Namely, we have

$$
\begin{aligned}
\int_{\mathbb{R}}\left(\Lambda^{\sigma} \mathrm{v}\right) \Lambda^{\sigma}\left(\mathrm{v} \partial_{Y} \mathrm{v}\right) d Y & =\int_{\mathbb{R}}\left(\Lambda^{\sigma} \mathrm{v}\right) \mathrm{v} \partial_{Y}\left(\Lambda^{\sigma} \mathrm{v}\right) d Y+\int_{\mathbb{R}}\left(\Lambda^{\sigma} \mathrm{v}\right)\left[\Lambda^{\sigma}, \mathrm{v} \partial_{Y}\right] \mathrm{v} d Y \\
& \leqslant-\frac{1}{2} \int_{\mathbb{R}}\left(\partial_{Y} \mathrm{v}\right)\left(\Lambda^{\sigma} \mathrm{v}\right)^{2} d Y+C\left\|\partial_{Y} \mathrm{v}\right\|_{L^{\infty}}\|\mathrm{v}\|_{H^{\sigma}}^{2} \leqslant C\|\mathrm{v}\|_{H^{\sigma}}^{2}
\end{aligned}
$$

where we have integrated by parts the first term, and we have used (A.4) to estimate the commutator and the uniform bound on $(\tilde{\rho}, \tilde{u})$ in $H^{s} \hookrightarrow W^{1, \infty}$ (for $s \geqslant 2$ ). For the last integral, we integrate by parts in space, substitute $\partial_{T} \mathrm{v}-\eta \mathfrak{c} \varrho^{-1} \mathrm{v} \partial_{Y} \mathrm{~V}-\partial_{Y} G$ for $2 \mathfrak{c} \partial_{Y} \mathrm{~V}$ and integrate in time. This yields

$$
\begin{aligned}
\left\|\Lambda^{\sigma} \mathrm{v}\right\|_{L^{2}}^{2} \leqslant & \left\|\Lambda^{\sigma}\left(\hat{\rho}^{\mathrm{in}}-\frac{\varrho}{\mathfrak{c}} \hat{u}^{\mathrm{in}}\right)\right\|_{L^{2}}^{2}+C \eta \int_{0}^{T}\|\mathrm{v}\|_{H^{\sigma}}^{2} d \tau \\
& +\frac{1}{\mathfrak{c}} \int_{0}^{T} \int_{\mathbb{R}} \Lambda^{\sigma}\left(-\partial_{\tau} \mathrm{v}+\eta \frac{\mathfrak{c}}{\varrho} \mathrm{v} \partial_{Y} \mathrm{v}+\partial_{Y} G\right) \Lambda^{\sigma} G d Y d \tau,
\end{aligned}
$$


and here again $\int_{\mathbb{R}} \Lambda^{\sigma}\left(\partial_{Y} G\right) \Lambda^{\sigma} G d Y=0$. We use the commutator estimate A.4 and Young's inequality to deduce

$$
\begin{aligned}
\frac{\eta}{\varrho} \int_{\mathbb{R}} \Lambda^{\sigma}\left(\mathrm{v} \partial_{Y} \mathrm{v}\right) \Lambda^{\sigma} G d Y & \leqslant \frac{\eta}{\varrho} \int_{\mathbb{R}} \mathrm{v}\left(\Lambda^{\sigma} \partial_{Y} \mathrm{v}\right) \Lambda^{\sigma} G d Y+C \eta\left\|\partial_{Y} \mathrm{v}\right\|_{L^{\infty}}\|\mathrm{v}\|_{H^{\sigma}}\left\|\Lambda^{\sigma} G\right\|_{L^{2}} \\
& \leqslant C \eta\|\mathrm{v}\|_{L^{2}}\left\|\Lambda^{\sigma} \partial_{Y} \mathrm{v}\right\|_{L^{\infty}}\left\|\Lambda^{\sigma} G\right\|_{L^{2}}+C \eta\|\mathrm{v}\|_{H^{\sigma}}\|G\|_{H^{\sigma}} \\
& \leqslant C \eta\|\mathrm{v}\|_{H^{\sigma}}\left(\eta+\varepsilon^{2}\right) \leqslant C \eta\|\mathrm{v}\|_{H^{\sigma}}^{2}+\eta\left(\eta+\varepsilon^{2}\right)^{2} .
\end{aligned}
$$

Here above, we have also used that $\sigma \leqslant s-3$, so that $\Lambda^{\sigma} \partial_{Y} \mathrm{v}$ is uniformly bounded in $H^{s-\sigma-1} \subset L^{\infty}$ and $\|G\|_{H^{\sigma}} \leqslant C\left(\eta+\varepsilon^{2}\right)$. Therefore, integrating by parts in time the remaining integral in 25 , we deduce, for $0 \leqslant T \leqslant T_{*} / \eta$,

$$
\begin{aligned}
\left\|\Lambda^{\sigma} \mathrm{v}\right\|_{L^{2}}^{2} \leqslant & \left\|\hat{\rho}^{\mathrm{in}}-\frac{\varrho}{\mathfrak{c}} \hat{u}^{\mathrm{in}}\right\|_{H^{\sigma}}^{2}+\frac{1}{\mathfrak{c}} \int_{\mathbb{R}}\left[\Lambda^{\sigma} \mathrm{v}(0) \Lambda^{\sigma} G(0)-\Lambda^{\sigma} \mathrm{v}(T) \Lambda^{\sigma} G(T)\right] d Y \\
& +\frac{1}{\mathfrak{c}} \int_{0}^{T} \int_{\mathbb{R}} \Lambda^{\sigma} \mathrm{v} \Lambda^{\sigma} \partial_{\tau} G d Y d \tau+C \eta \int_{0}^{T}\|\mathrm{v}\|_{H^{\sigma}}^{2} d \tau+C\left(\eta+\varepsilon^{2}\right)^{2} \\
\leqslant & C\left\|\hat{\rho}^{\mathrm{in}}-\frac{\varrho}{\mathfrak{c}} \hat{u}^{\mathrm{in}}\right\|_{H^{\sigma}}^{2}+\frac{1}{4}\left\|\Lambda^{\sigma} \mathrm{v}\right\|_{H^{\sigma}}^{2} \\
& +\frac{1}{\mathfrak{c}} \int_{0}^{T} \int_{\mathbb{R}} \Lambda^{\sigma} \mathrm{v} \Lambda^{\sigma} \partial_{\tau} G d Y d \tau+C \eta \int_{0}^{T}\|\mathrm{v}\|_{H^{\sigma}}^{2} d \tau+C\left(\eta+\varepsilon^{2}\right)^{2}
\end{aligned}
$$

where we have used that $\|G\|_{H^{\sigma}} \leqslant C\left(\eta+\varepsilon^{2}\right)$, since $\sigma-3 \leqslant s$. From the first equation in (19) we have $\partial_{\tau} \tilde{\rho}-\mathfrak{c} \partial_{Y} \mathrm{v}=-\eta \partial_{Y}(\tilde{\rho} \tilde{u})$, thus

$$
\left\|\partial_{\tau} \tilde{\rho}-\mathfrak{c} \partial_{Y} \mathrm{v}\right\|_{H^{s-1}} \leqslant C \eta,
$$

and this implies, since $H^{s-3}$ is an algebra (recall that $s>7 / 2$ ) and $\sigma \leqslant s-3$,

$$
\begin{aligned}
\partial_{\tau} G= & \frac{\varrho}{\mathfrak{c}}\left(g^{\prime}(\varrho+\eta \tilde{\rho})-g^{\prime}(\varrho)\right) \partial_{\tau} \tilde{\rho}-\eta \frac{\mathfrak{c}}{\varrho} \tilde{\rho} \partial_{\tau} \tilde{\rho} \\
& \quad-\varepsilon^{2} \frac{\varrho}{\mathfrak{c}}\left(K(\varrho+\eta \tilde{\rho}) \partial_{Y}^{2} \partial_{\tau} \tilde{\rho}+\eta K^{\prime}(\varrho+\eta \tilde{\rho}) \partial_{\tau} \tilde{\rho} \partial_{Y}^{2} \tilde{\rho}\right. \\
& \left.\quad+\eta K^{\prime}(\varrho+\eta \tilde{\rho}) \partial_{Y} \tilde{\rho} \partial_{Y} \partial_{\tau} \tilde{\rho}+\frac{\eta^{2}}{2} K^{\prime \prime}(\varrho+\eta \tilde{\rho})\left(\partial_{Y} \tilde{\rho}\right)^{2} \partial_{\tau} \tilde{\rho}\right) \\
= & \varrho\left(g^{\prime}(\varrho+\eta \tilde{\rho})-g^{\prime}(\varrho)\right) \partial_{Y} \mathrm{v}-\eta \frac{\mathfrak{c}^{2}}{\varrho} \tilde{\rho} \partial_{Y} \mathrm{v}-\varepsilon^{2} \varrho K(\varrho) \partial_{Y}^{3} \mathrm{~V}+\mathcal{O}_{L^{\infty}\left(\left[0, T_{*} / \eta\right], H^{\sigma}\right)}\left(\eta\left(\varepsilon^{2}+\eta\right)\right) .
\end{aligned}
$$

We then deduce by Young's inequality again that

$$
\begin{aligned}
\frac{3}{4}\left\|\Lambda^{\sigma} \mathrm{v}\right\|_{L^{2}}^{2} \leqslant & C\left\|\hat{\rho}^{\text {in }}-\frac{\varrho}{\mathfrak{c}} \hat{u}^{\mathrm{in}}\right\|_{H^{\sigma}}^{2}+C\left(\eta+\varepsilon^{2}\right)^{2}+C \eta \int_{0}^{T}\|\mathrm{v}\|_{H^{\sigma}}^{2} d \tau \\
& +\frac{1}{\mathfrak{c}} \int_{0}^{T} \int_{\mathbb{R}} \Lambda^{\sigma}\left(\varrho\left(g^{\prime}(\varrho+\eta \tilde{\rho})-g^{\prime}(\varrho)\right) \partial_{Y} \mathrm{v}-\eta \frac{\mathfrak{c}^{2}}{\varrho} \tilde{\rho} \partial_{Y} \mathrm{v}-\varepsilon^{2} \varrho K(\varrho) \partial_{Y^{3}}^{3} \mathrm{v}\right) \Lambda^{\sigma} \mathrm{v} d Y d \tau \\
\leqslant & C\left\|\hat{\rho}^{\text {in }}-\frac{\varrho}{\mathfrak{c}} \hat{u}^{\text {in }}\right\|_{H^{\sigma}}^{2}+C\left(\eta+\varepsilon^{2}\right)^{2}+C \eta \int_{0}^{T}\|\mathrm{v}\|_{H^{\sigma}}^{2} d \tau
\end{aligned}
$$


by computations similar to those of Lemma 1 and by using the tame estimate (A.4). The conclusion then follows from Gronwall's lemma.

Our next result is an improvement of the previous one in terms of loss of derivatives. The idea is to apply $\partial_{T}$ to the equation and estimate $\partial_{T}(\tilde{\rho}, \tilde{u})$, as in [11]. Since the equations in (19) are formally of the form $\partial_{T} \tilde{\rho}-\mathfrak{c} \partial_{Y} \mathrm{~V}=\mathcal{O}(\eta)$ and $\partial_{T} \tilde{u}+\mathfrak{c} \varrho^{-1} \partial_{Y} \mathrm{~V}=\mathcal{O}\left(\eta+\varepsilon^{2}\right)$, where the remainder terms involve at most three derivatives, this will allow to establish a better estimate, losing only two derivatives instead of three.

Lemma 3 We assume that $s>7 / 2$. Then, in the framework of Theorem 4, the following estimates hold true.

For any $3 / 2<\sigma \leqslant s-2$, there exists $C$, depending only on $s, \sigma$ and $M$, so that

$$
\sup _{0 \leqslant T \leqslant T_{*} / \eta}\left\|\tilde{\rho}-\frac{\varrho}{\mathfrak{c}} \tilde{u}\right\|_{H^{\sigma}} \leqslant C\left(\left\|\hat{\rho}^{\text {in }}-\frac{\varrho}{\mathfrak{c}} \hat{u}^{\text {in }}\right\|_{H^{\sigma}}+\eta+\varepsilon^{2}\right) .
$$

For every $3 / 2<\sigma \leqslant s-1$, there exists $C$, depending only on $s, \sigma$ and $M$, so that

$$
\sup _{0 \leqslant T \leqslant T_{*} / \eta}\left\|\tilde{\rho}-\frac{\varrho}{\mathfrak{c}} \tilde{u}\right\|_{H^{\sigma}} \leqslant C\left(\left\|\hat{\rho}^{\text {in }}-\frac{\varrho}{\mathfrak{c}} \hat{u}^{\text {in }}\right\|_{H^{\sigma}}+\eta+\varepsilon\right) .
$$

Note that the second estimate here above may be poorer than the first one (if $\eta \ll \varepsilon$ ) in terms of the error value, but it controls one more derivative.

Proof. The starting point is the system $(19)$ written with the complex extended formulation, namely

$$
\partial_{T} \tilde{z}-\mathfrak{c} \partial_{Y} \tilde{z}+\eta \tilde{u} \partial_{Y} \tilde{z}+i \eta\left(\partial_{Y} \tilde{z}\right) \tilde{w}+\frac{1}{\varepsilon} b(\rho) \tilde{w}+i \varepsilon \partial_{Y}\left(a(\rho) \partial_{Y} \tilde{z}\right)=0 .
$$

Using the uniform bounds for $\left(\tilde{u}, \partial_{Y} \tilde{z}, \tilde{w}\right)$ in the algebra $H^{s-1}(s>7 / 2)$, we infer

$$
\left\|\eta \tilde{u} \partial_{Y} \tilde{z}+i \eta\left(\partial_{Y} \tilde{z}\right) \tilde{w}\right\|_{H^{\sigma-1}} \leqslant C \eta \text {. }
$$

In addition, since $H^{\sigma}$ is an algebra $(\sigma>3 / 2)$,

$$
\begin{aligned}
\left\|\varepsilon \partial_{Y}\left(a(\rho) \partial_{Y} \tilde{w}\right)\right\|_{H^{\sigma-1}} & \leqslant \varepsilon^{2}\left\|a(\rho) \partial_{Y}\left(g^{\prime}(\rho) \partial_{Y} \tilde{\rho} / b(\rho)\right)\right\|_{H^{\sigma}} \\
& \leqslant C \varepsilon^{2}\left\|g^{\prime}(\rho) \partial_{Y} \tilde{\rho} / b(\rho)\right\|_{H^{\sigma+1}} \\
& \leqslant C \varepsilon^{2}\left\|\partial_{Y} \tilde{\rho}\right\|_{H^{\sigma+1}} \\
& \leqslant \begin{cases}C \varepsilon^{2} & \text { if } \sigma+2 \leqslant s \\
C \varepsilon & \text { if } \sigma+1 \leqslant s .\end{cases}
\end{aligned}
$$

We pursue the computations in the case $\sigma \leqslant s-2$ and point out the modifications to make when $\sigma \leqslant s-1$. Then, we have

$$
\begin{aligned}
0 & =\partial_{T} \tilde{z}-\mathfrak{c} \partial_{Y} \tilde{z}+\frac{1}{\varepsilon} b(\rho) \tilde{w}+i \varepsilon \partial_{Y}\left(a(\rho) \partial_{Y} \tilde{u}\right)+\mathcal{O}_{L^{\infty}\left(\left[0, T_{*} / \eta\right], H^{\sigma-1}\right)}\left(\eta+\varepsilon^{2}\right) \\
& =\partial_{T} \tilde{z}-\mathfrak{c} \partial_{Y} \tilde{u}+g^{\prime}(\rho) \partial_{Y} \tilde{\rho}-i \varepsilon \partial_{Y}\left(\mathfrak{c} b(\rho)^{-1} g^{\prime}(\rho) \partial_{Y} \tilde{\rho}-a(\rho) \partial_{Y} \tilde{u}\right)+\mathcal{O}_{L^{\infty}\left(\left[0, T_{*} / \eta\right], H^{\sigma-1}\right)}\left(\eta+\varepsilon^{2}\right) \\
& =\partial_{T} \tilde{z}-\mathfrak{c} \partial_{Y} \tilde{u}+g^{\prime}(\varrho) \partial_{Y} \tilde{\rho}-i \varepsilon \partial_{Y}\left(\mathfrak{c} b(\varrho)^{-1} g^{\prime}(\varrho) \partial_{Y} \tilde{\rho}-a(\varrho) \partial_{Y} \tilde{u}\right)+\mathcal{O}_{L^{\infty}\left(\left[0, T_{*} / \eta\right], H^{\sigma-1}\right)}\left(\eta+\varepsilon^{2}\right),
\end{aligned}
$$


using that, since $H^{s-1}$ is an algebra, we have

$$
\begin{gathered}
\left\|\left(g^{\prime}(\rho)-g^{\prime}(\varrho)\right) \partial_{Y} \tilde{\rho}\right\|_{H^{\sigma}} \leqslant\left\|\left(g^{\prime}(\rho)-g^{\prime}(\varrho)\right) \partial_{Y} \tilde{\rho}\right\|_{H^{s-1}} \leqslant C \eta \\
\left\|(a(\varrho)-a(\rho)) \partial_{Y} \tilde{u}\right\|_{H^{\sigma}} \leqslant\left\|(a(\varrho)-a(\rho)) \partial_{Y} \tilde{u}\right\|_{H^{s-1}} \leqslant C \eta
\end{gathered}
$$

and finally

$$
\left\|\left(\mathfrak{c} b(\rho)^{-1} g^{\prime}(\rho)-\mathfrak{c} b(\varrho)^{-1} g^{\prime}(\varrho)\right) \partial_{Y} \tilde{u}\right\|_{H^{\sigma}} \leqslant C \eta .
$$

(If $\sigma \leqslant s-1$, the error $\eta+\varepsilon^{2}$ is replaced by $\eta+\varepsilon$ and the term

$$
\left\|\varepsilon \partial_{Y}\left(\mathfrak{c} b(\varrho)^{-1} g^{\prime}(\varrho) \partial_{Y} \tilde{\rho}-a(\varrho) \partial_{Y} \tilde{u}\right)\right\|_{H^{\sigma-1}} \leqslant \varepsilon\left\|\mathfrak{c} b(\varrho)^{-1} g^{\prime}(\varrho) \partial_{Y} \tilde{\rho}-a(\varrho) \partial_{Y} \tilde{u}\right\|_{H^{s-1}} \leqslant C \varepsilon
$$

can be incorporated into the remainder.) Since $a(\varrho) b(\varrho)=\varrho g^{\prime}(\varrho)=\mathfrak{c}^{2}$, we deduce

$$
\begin{aligned}
0 & =\partial_{T} \tilde{z}+\mathfrak{c}^{2} \varrho^{-1} \partial_{Y}\left(\tilde{\rho}-\varrho \mathfrak{c}^{-1} \tilde{u}\right)-i \varepsilon \frac{\mathfrak{c} a(\varrho)}{\varrho} \partial_{Y}^{2}\left(\tilde{\rho}-\varrho \mathfrak{c}^{-1} \tilde{u}\right)+\mathcal{O}_{L^{\infty}\left(\left[0, T_{*} / \eta\right], H^{\sigma-1}\right)}\left(\eta+\varepsilon^{2}\right) \\
& =\partial_{T} \tilde{z}+\mathfrak{c}^{2} \varrho^{-1} \partial_{Y} \mathrm{v}-i \varepsilon \frac{\mathfrak{c} a(\varrho)}{\varrho} \partial_{Y}^{2} \mathrm{v}+\mathcal{O}_{L^{\infty}\left(\left[0, T_{*} / \eta\right], H^{\sigma-1}\right)}\left(\eta+\varepsilon^{2}\right)
\end{aligned}
$$

In particular, for $T=0$,

$$
\left\|\left(\partial_{T} \tilde{z}\right)_{\mid T=0}\right\|_{H^{\sigma-1}} \leqslant C\left\|\partial_{Y} \mathrm{v}_{\mid T=0}\right\|_{H^{\sigma-1}}+C \varepsilon\left\|\partial_{Y}^{2} \mathrm{v}_{\mid T=0}\right\|_{H^{\sigma-1}}+C\left(\eta+\varepsilon^{2}\right) .
$$

Since $\sigma \leqslant s-2$, we may now use the interpolation and Young's inequalities

$$
\varepsilon\left\|\partial_{Y}^{2} \mathrm{v}\right\|_{H^{\sigma-1}} \leqslant \varepsilon\|\mathrm{v}\|_{H^{\sigma}}^{1 / 2}\|\mathrm{v}\|_{H^{\sigma+2}}^{1 / 2} \leqslant C\|\mathrm{v}\|_{H^{\sigma}}+C \varepsilon^{2}
$$

to infer

$$
\left\|\left(\partial_{T} \tilde{z}\right)_{\mid T=0}\right\|_{H^{\sigma-1}} \leqslant C\left\|\mathrm{v}_{\mid T=0}\right\|_{H^{\sigma}}+C\left(\eta+\varepsilon^{2}\right) .
$$

When $\sigma \leqslant s-1$, the error is $\mathcal{O}(\eta+\varepsilon)$ and $\varepsilon\left\|\partial_{Y}^{2} \mathrm{v}\right\|_{H^{\sigma-1}}=\mathcal{O}(\varepsilon)$ since $\sigma+1 \leqslant s$.

Applying $\partial_{T}$ to the equation for $\tilde{z}$, we see that $\tilde{z}_{T} \stackrel{\text { def }}{=} \partial_{T} \tilde{z}$ solves

$$
\begin{aligned}
\partial_{T} \tilde{z}_{T}-\mathfrak{c} \partial_{Y} \tilde{z}_{T}+ & \eta \tilde{u} \partial_{Y} \tilde{z}_{T}+i \eta\left(\partial_{Y} \tilde{z}_{T}\right) \tilde{w}+\frac{1}{\varepsilon} b(\rho) \tilde{w}_{T}+i \varepsilon \partial_{Y}\left(a(\rho) \partial_{Y} \tilde{z}_{T}\right) \\
& =-\eta \tilde{z}_{T} \partial_{Y} \tilde{z}-\frac{\eta}{\varepsilon} b^{\prime}(\rho) \partial_{T} \tilde{\rho} \tilde{w}-i \varepsilon \eta \partial_{Y}\left(a^{\prime}(\rho) \partial_{T} \tilde{\rho} \partial_{Y} \tilde{z}\right)
\end{aligned}
$$

and we wish to perfom an $H^{\sigma-1}$ estimate, with $\sigma-1>1 / 2$. We first easily bound some source terms by using that $H^{\sigma-1}$ is an algebra:

$$
\left\|\eta \tilde{z}_{T} \partial_{Y} \tilde{z}\right\|_{H^{\sigma-1}} \leqslant C \eta\left\|\tilde{z}_{T}\right\|_{H^{\sigma-1}}\left\|\partial_{Y} \tilde{z}\right\|_{H^{\sigma-1}} \leqslant C \eta\left\|\tilde{z}_{T}\right\|_{H^{\sigma-1}} .
$$

In view of the equation $\partial_{T} \tilde{\rho}=\mathfrak{c} \partial_{Y} \mathrm{~V}-\eta \partial_{Y}(\tilde{\rho} \tilde{u})$ we obtain $(\sigma+1 \leqslant s)$ on the one hand

$$
\left\|\frac{\eta}{\varepsilon} b^{\prime}(\rho)\left(\partial_{T} \tilde{\rho}-\mathfrak{c} \partial_{Y} \mathrm{v}\right) \tilde{w}\right\|_{H^{\sigma-1}} \leqslant C \frac{\eta^{2}}{\varepsilon}\|\tilde{\rho} \tilde{u}\|_{H^{\sigma}}\|\tilde{w}\|_{H^{\sigma-1}} \leqslant C \eta^{2}
$$


and on the other hand

$$
\left\|\varepsilon \eta \partial_{Y}\left(a^{\prime}(\rho)\left(\partial_{T} \tilde{\rho}-\mathfrak{c} \partial_{Y} \mathrm{v}\right) \partial_{Y} \tilde{z}\right)\right\|_{H^{\sigma-1}} \leqslant C \varepsilon \eta^{2}\|\tilde{\rho} \tilde{u}\|_{H^{\sigma+1}}\left\|\partial_{Y} \tilde{z}\right\|_{H^{\sigma}} \leqslant C \varepsilon \eta^{2} .
$$

We now express, by the second equation in (19), $\partial_{Y} \mathrm{v}$ in terms of the real part of $\tilde{z}_{T}$ :

$$
\begin{aligned}
0 & =\partial_{T} \tilde{u}-\mathfrak{c} \partial_{Y} \tilde{u}+\eta \tilde{u} \partial_{Y} \tilde{u}+g^{\prime}(\varrho+\eta \tilde{\rho}) \partial_{Y} \tilde{\rho}-\varepsilon^{2} \partial_{Y}\left(K(\varrho+\eta \tilde{\rho}) \partial_{Y}^{2} \tilde{\rho}+\frac{\eta}{2} K^{\prime}(\varrho+\eta \tilde{\rho})\left(\partial_{Y} \tilde{\rho}\right)^{2}\right) \\
& =\operatorname{Re}\left(\tilde{z}_{T}\right)+\mathfrak{c}^{2} \varrho^{-1} \partial_{Y} \mathrm{v}+\mathcal{O}_{L^{\infty}\left(\left[0, T_{*} / \eta\right], H^{\sigma-1}\right)}\left(\eta+\varepsilon^{2}\right)
\end{aligned}
$$

since $\sigma+2 \leqslant s$ (the error is $\mathcal{O}(\eta+\varepsilon)$ if $\sigma+1 \leqslant s$ ), and infer

$$
\begin{aligned}
-\frac{\eta}{\varepsilon} b^{\prime}(\rho) \partial_{T} \tilde{\rho} \tilde{w} & =\mathfrak{c} \varrho \frac{\eta}{\varepsilon} b^{\prime}(\rho) \operatorname{Re}\left(\tilde{z}_{T}\right) \tilde{w}+\mathcal{O}_{L^{\infty}\left(\left[0, T_{*} / \eta\right], H^{\sigma-1}\right)}\left(\eta\left(\eta+\varepsilon^{2}\right)\right) \\
& =\mathcal{O}_{H^{\sigma-1}}\left(\eta\left\|\tilde{z}_{T}\right\|_{H^{\sigma-1}}\right)+\mathcal{O}_{L^{\infty}\left(\left[0, T_{*} / \eta\right], H^{\sigma-1}\right)}\left(\eta\left(\eta+\varepsilon^{2}\right)\right)
\end{aligned}
$$

since $\tilde{w}=\mathcal{O}(\varepsilon)$ in $H^{s-1}$. Concerning the term

$$
\varepsilon \eta \partial_{Y}\left(a^{\prime}(\rho) \partial_{T} \tilde{\rho} \partial_{Y} \tilde{z}\right)=\varepsilon \eta \partial_{Y}\left(a^{\prime}(\rho) \partial_{Y} \tilde{z}\right) \partial_{T} \tilde{\rho}+\varepsilon \eta a^{\prime}(\rho) \partial_{Y} \tilde{z} \partial_{Y} \partial_{T} \tilde{\rho}
$$

in (28), the first term in the right-hand side satisfies

$$
\begin{gathered}
\left\|\varepsilon \eta \partial_{Y}\left(a^{\prime}(\rho) \partial_{Y} \tilde{z}\right) \partial_{T} \tilde{\rho}\right\|_{H^{\sigma-1}} \leqslant C \varepsilon \eta\left\|\partial_{Y}\left(a^{\prime}(\rho) \partial_{Y} \tilde{z}\right) \operatorname{Re}\left(\tilde{z}_{T}\right)\right\|_{H^{\sigma-1}}+C \varepsilon \eta^{2} \\
\leqslant C \eta\left\|\operatorname{Re}\left(\tilde{z}_{T}\right)\right\|_{H^{\sigma-1}}+C \varepsilon \eta^{2}
\end{gathered}
$$

For the second one, we write

$$
\begin{aligned}
\varepsilon \eta a^{\prime}(\rho) \partial_{Y} \tilde{z} \partial_{Y} \partial_{T} \tilde{\rho} & =\eta a^{\prime}(\rho) \partial_{Y} \tilde{z} \partial_{T}(\sqrt{\rho / K(\rho)} \tilde{w}) \\
& =\eta a^{\prime}(\rho) \partial_{Y} \tilde{z} \sqrt{\rho / K(\rho)} \operatorname{Im}\left(\tilde{z}_{T}\right)+\eta a^{\prime}(\rho) \partial_{Y} \tilde{z} \tilde{w} \partial_{T}(\sqrt{\rho / K(\rho)}) \\
& =\mathcal{O}_{H^{\sigma-1}}\left(\eta\left\|\tilde{z}_{T}\right\|_{H^{\sigma-1}}\right)+\mathcal{O}_{H^{\sigma-1}}\left(\eta^{2}\right) .
\end{aligned}
$$

Gathering all these estimates and inserting them in (28), we arrive at

$$
\begin{aligned}
\partial_{T} \tilde{z}_{T}-\mathfrak{c} \partial_{Y} \tilde{z}_{T}+ & \eta \tilde{u} \partial_{Y} \tilde{z}_{T}+i \eta\left(\partial_{Y} \tilde{z}_{T}\right) \tilde{w}+\frac{1}{\varepsilon} b(\rho) \tilde{w}_{T}+i \varepsilon \partial_{Y}\left(a(\rho) \partial_{Y} \tilde{z}_{T}\right) \\
& =\mathcal{O}_{H^{\sigma-1}}\left(\eta\left\|\tilde{z}_{T}\right\|_{H^{\sigma-1}}\right)+\mathcal{O}_{L^{\infty}\left(\left[0, T_{*} / \eta\right], H^{\sigma-1}\right)}\left(\eta\left(\eta+\varepsilon^{2}\right)\right)
\end{aligned}
$$

Therefore, following the lines of the proof of Theorem 2 , we infer, for $0 \leqslant T \leqslant T_{*} / \eta$, $\left\|\left(\partial_{T} \tilde{\rho}, \tilde{z}_{T}\right)\right\|_{H^{\sigma-1}} \leqslant C\left\|\left(\partial_{T} \tilde{\rho}, \tilde{z}_{T}\right)(T=0)\right\|_{H^{\sigma-1}}+C\left(\eta+\varepsilon^{2}\right) \leqslant C\left\|\tilde{z}_{T}(T=0)\right\|_{H^{\sigma-1}}+C\left(\eta+\varepsilon^{2}\right)$, since $\left(\partial_{T} \tilde{\rho}(T=0)=-\eta \partial_{X}(\tilde{\rho} \tilde{u})=\mathcal{O}_{H^{\sigma-1}}(\eta)\right.$. As a consequence, considering the real part of (27), we obtain

$$
\left\|\partial_{Y} \mathrm{v}\right\|_{H^{\sigma-1}} \leqslant C\left\|\operatorname{Re}\left(\tilde{z}_{T}\right)\right\|_{H^{\sigma-1}}+C\left(\eta+\varepsilon^{2}\right) \leqslant C\left\|\tilde{\rho}^{\text {in }}-\varrho \mathfrak{c}^{-1} \tilde{u}^{\text {in }}\right\|_{H^{\sigma}}+C\left(\eta+\varepsilon^{2}\right),
$$

which completes the proof (since the $L^{2}$ norm of $\mathrm{v}$ is already estimated in Lemma 1 ). 
Proof of Theorem 4 completed. We focus first on (ii) in the case $s \geqslant 5$ and $0 \leqslant \sigma \leqslant s-5$. In particular, we may apply Lemma 3 with $3 / 2<\sigma+3 \leqslant s-2$ to deduce

$$
\|\mathrm{v}\|_{H^{\sigma+3}} \leqslant C\left(\eta+\varepsilon^{2}+\left\|\hat{\rho}^{\text {in }}-\varrho c^{-1} \hat{u}^{\text {in }}\right\|_{H^{\sigma+3}}\right) .
$$

Combining the two equations in 19 , we infer that

$$
\mathrm{w} \stackrel{\text { def }}{=} \frac{1}{2}\left(\tilde{\rho}+\frac{\varrho}{\mathfrak{c}} \tilde{u}\right)
$$

satisfies

$$
\begin{aligned}
2 \partial_{T} \mathrm{w}+2 \eta \Gamma \mathrm{w} \partial_{Y} \mathrm{w}-2 \varepsilon^{2} \kappa \partial_{Y}^{3} \mathrm{w}= & -\frac{\varrho}{\mathfrak{c}}\left[g^{\prime}(\varrho+\eta \tilde{\rho})-g^{\prime}(\varrho)-\eta g^{\prime \prime}(\varrho) \tilde{\rho}\right] \partial_{Y} \tilde{\rho} \\
& +\eta\left(\frac{\mathfrak{c}}{\varrho}-\frac{\varrho}{\mathfrak{c}} g^{\prime \prime}(\varrho)\right) \mathrm{v} \partial_{Y} \mathrm{w}+\eta\left(\frac{\mathfrak{c}}{\varrho}-\frac{\varrho}{\mathfrak{c}} g^{\prime \prime}(\varrho)\right) \tilde{\rho} \partial_{Y} \mathrm{v}+2 \varepsilon^{2} \kappa \partial_{Y}^{3} \mathrm{v} \\
& +\varepsilon^{2} \frac{\varrho}{2 \mathfrak{c}} \partial_{Y}\left([K(\varrho+\eta \tilde{\rho})-K(\varrho)] \partial_{Y}^{2} \tilde{\rho}+\frac{\eta}{2} K^{\prime}(\varrho+\eta \tilde{\rho})\left(\partial_{Y} \tilde{\rho}\right)^{2}\right) .
\end{aligned}
$$

From the $H^{\sigma+3}$ bound on $\mathrm{v}$ and the uniform bounds of Theorem 2, we infer consistency with the KdV equation by using A.1. Our consistency estimate reads

$$
\left\|\partial_{T^{\mathrm{w}}}+\eta \Gamma \mathrm{w} \partial_{Y} \mathrm{w}-\varepsilon^{2} \kappa \partial_{Y}^{3} \mathrm{w}\right\|_{H^{\sigma}} \leqslant C\left(\eta+\varepsilon^{2}\right)\left(\eta+\varepsilon^{2}+\left\|\hat{\rho}^{\mathrm{in}}-\frac{\varrho}{\mathfrak{c}} \hat{u}^{\mathrm{in}}\right\|_{H^{\sigma+3}}\right) .
$$

for $0 \leqslant \sigma \leqslant s-5$, and allows by very standard estimates on the KdV equation - like the one in (24) - to conclude that

$$
\|\mathrm{w}-\zeta(\eta T, \cdot-\mathfrak{c} T)\|_{H^{\sigma}(\mathbb{R})} \leqslant C\left(1+\frac{\varepsilon^{2}}{\eta}\right)\left(\eta+\varepsilon^{2}+\left\|\hat{\rho}^{\mathrm{in}}-\frac{\varrho}{\mathfrak{c}} \hat{u}^{\mathrm{in}}\right\|_{H^{\sigma+3}(\mathbb{R})}\right) .
$$

Then it suffices to write

$$
\tilde{\rho}=\frac{\mathrm{v}+\mathrm{w}}{2} \quad \text { and } \quad \tilde{u}=\frac{\mathfrak{c}}{2 \varrho}(\mathrm{w}-\mathrm{v})
$$

and use Lemma 3 to complete the proof of the second statement of $(i i)$. If $s \geqslant 4$, we use the second estimate in Lemma 3 ,

We now turn to $(i)$ in the case $s \geqslant 4$ (or $s>9 / 2$ ), and write (30) under the form

$$
\begin{aligned}
2 \partial_{T} \mathrm{w}+2 \eta \Gamma \mathrm{w} \partial_{Y} \mathrm{w}= & -\frac{\varrho}{\mathfrak{c}}\left[g^{\prime}(\varrho+\eta \tilde{\rho})-g^{\prime}(\varrho)-\eta g^{\prime \prime}(\varrho) \tilde{\rho}\right] \partial_{Y} \tilde{\rho} \\
& +\eta\left(\frac{\mathfrak{c}}{\varrho}-\frac{\varrho}{\mathfrak{c}} g^{\prime \prime}(\varrho)\right) \mathrm{v} \partial_{Y} \mathrm{w}+\eta\left(\frac{\mathfrak{c}}{\varrho}-\frac{\varrho}{\mathfrak{c}} g^{\prime \prime}(\varrho)\right) \tilde{\rho} \partial_{Y} \mathrm{v} \\
& +\varepsilon^{2} \frac{\varrho}{2 \mathfrak{c}} \partial_{Y}\left(K(\varrho+\eta \tilde{\rho}) \partial_{Y}^{2} \tilde{\rho}+\frac{\eta}{2} K^{\prime}(\varrho+\eta \tilde{\rho})\left(\partial_{Y} \tilde{\rho}\right)^{2}\right),
\end{aligned}
$$

with the dispersive terms in the source terms. Note also that if ( $s \geqslant 4$ and $\sigma \in[0, s-4])$, or if $(s>9 / 2$ and $\sigma \in[0, s-3])$, then Lemma 2 or Lemma 3 with $3 / 2<\sigma+1 \leqslant s-2$ gives

$$
\|\mathrm{v}\|_{H^{\sigma+1}} \leqslant C\left(\eta+\varepsilon^{2}+\left\|\rho^{\text {in }}-\varrho c^{-1} \hat{u}^{\text {in }}\right\|_{H^{\sigma+1}}\right) .
$$


From this, we infer the consistency estimate

$$
\left\|\partial_{T} \mathrm{w}+\eta \Gamma \mathrm{w} \partial_{Y} \mathrm{w}\right\|_{H^{\sigma}} \leqslant C \eta\left(\eta+\frac{\varepsilon^{2}}{\eta}+\left\|\hat{\rho}^{\text {in }}-\frac{\varrho}{\mathfrak{c}} \hat{u}^{\text {in }}\right\|_{H^{\sigma+1}}\right) .
$$

In the case $(s \geqslant 3$ and $\sigma \in[0, s-3])$, or $(s>7 / 2$ and $\sigma \in[0, s-2])$, we have

$$
\|\mathrm{v}\|_{H^{\sigma+1}} \leqslant C\left(\eta+\varepsilon+\left\|\hat{\rho}^{\text {in }}-\varrho c^{-1} \hat{u}^{\text {in }}\right\|_{H^{\sigma+1}}\right)
$$

and

$$
\left\|\partial_{T} \mathrm{w}+\eta \Gamma \mathrm{w} \partial_{Y} \mathrm{w}\right\|_{H^{\sigma}} \leqslant C \eta\left(\eta+\varepsilon+\frac{\varepsilon^{2}}{\eta}+\left\|\hat{\rho}^{\text {in }}-\frac{\varrho}{\mathfrak{c}} \hat{u}^{\text {in }}\right\|_{H^{\sigma+1}}\right)
$$

which completes the proof.

\subsection{The $\mathrm{KdV}$ regime for travelling waves}

Under fairly general assumptions on the energy density $\mathscr{F}$, (gEK) admits rich families of planar travelling wave solutions. Indeed, for $(\rho, u)=(R, U)(x-\sigma t)$ to solve the one $\mathrm{D}$ version of $(\mathrm{gEK})$ the profile $(R, U)$ must solve the ODEs

$$
\left\{\begin{array}{l}
(R(U-\sigma))^{\prime}=0 \\
\left.\left(\frac{1}{2}(U-\sigma)^{2}+\delta \mathscr{F}[R]\right)\right)^{\prime}=0
\end{array}\right.
$$

which is equivalent to the existence of three constants $(j, \lambda, \mu)$ such that

$$
\left\{\begin{array}{l}
R(U-\sigma)=j \\
R^{\prime} \frac{\partial \mathscr{F}}{\partial \rho_{x}}\left(R, R^{\prime}\right)-\mathscr{F}\left(R, R^{\prime}\right)+\frac{j^{2}}{2 R}+\lambda R=\mu
\end{array}\right.
$$

(see [3] for more details). If $\varrho>0$ and $(j, \lambda)$ are such that $(\varrho, 0)$ is a strict local minimum of the mapping $\mathscr{H}:(R, \dot{R}) \mapsto \dot{R} \frac{\partial \mathscr{F}}{\partial \rho_{x}}(R, \dot{R})-\mathscr{F}(R, \dot{R})+\frac{j^{2}}{2 R}+\lambda R$ then the level sets

$$
\left\{(R, \dot{R}) ; \dot{R} \frac{\partial \mathscr{F}}{\partial \rho_{x}}(R, \dot{R})-\mathscr{F}(R, \dot{R})+\frac{j^{2}}{2 R}+\lambda R=\mu\right\}
$$

consist of closed curves for $\mu$ greater than, and close to $-\mathscr{F}(\varrho, 0)+\frac{j^{2}}{2 \varrho}+\lambda \varrho$. These correspond to periodic travelling wave solutions to $(\mathrm{gEK})$ oscillating around $\varrho$. Note that, since the Hessian matrix of $\mathscr{H}$ at $(\varrho, 0)$ is given by

$$
\operatorname{Hess} \mathscr{H}(\varrho, 0)=\left(\begin{array}{cc}
-\frac{\partial^{2} \mathscr{F}}{\partial \rho^{2}}(\varrho, 0)+\frac{j^{2}}{\varrho^{3}} & 0 \\
0 & \frac{\partial^{2} \mathscr{F}}{\partial \rho_{x}^{2}}(\varrho, 0)
\end{array}\right)=\left(\begin{array}{cc}
\frac{j^{2}}{\varrho^{3}}-\frac{\mathfrak{c}^{2}}{\varrho^{2}} & 0 \\
0 & \underline{K}
\end{array}\right)
$$


the strict local minimization condition for $\mathscr{H}$ at $(\varrho, 0)$ is ensured by the inequalities $\underline{K}>0$, $j^{2}>\varrho^{2} \mathfrak{c}^{2}$, provided that $(\varrho, 0)$ is a critical point of $\mathscr{H}$, which requires that

$$
\lambda=\frac{\partial \mathscr{F}}{\partial \rho}(\varrho, 0)+\frac{j^{2}}{2 \varrho^{2}} .
$$

This means that (gEK) admits periodic travelling waves solutions with large enough momentum in the frame attached to them. Solitary waves with endstate $\varrho$ arise when $(\varrho, 0)$ is a saddle-point of $\mathscr{H}$. They are of small amplitude if this saddle-point is close to local minimum of $\mathscr{H}$. This happens only if $(\varrho, 0)$ is close to a critical point of $\mathscr{H}$ where the Hessian of $\mathscr{H}$ is singular. In other words, small amplitude solitary waves occur when $\varrho^{2} \mathfrak{c}^{2}-j^{2}$ is positive and close to zero. Note that for small amplitude waves around $(\varrho, 0)$, we have $j=R(U-\sigma) \approx-\varrho \sigma$, so that $\varrho^{2} \mathfrak{c}^{2}-j^{2}$ being close to zero is equivalent to $\sigma^{2}$ being close to $\mathfrak{c}^{2}$.

Let us consider a travelling wave $(\rho, u)(x, t)=(R, U)(x-\sigma t)$ solution to (gEK), of small amplitude around some reference state $(\varrho, 0)$. Assume moreover that its speed $\sigma$ is close to $\mathfrak{c}$, say $\sigma=\mathfrak{c}+\varepsilon^{2} \tilde{\sigma}$ with $\varepsilon>0$ small. Then of course we can write

$$
x-\sigma t=\left(\varepsilon(x-\mathfrak{c} t)-\tilde{\sigma} \varepsilon^{3} t\right) / \varepsilon,
$$

so that if we use the KdV rescaling in (1),

$$
(\rho, u)(x, t)=(\varrho, 0)+\varepsilon^{2}(\tilde{\rho}, \tilde{u})\left(\varepsilon(x-\mathfrak{c} t), \varepsilon^{3} t\right),
$$

we have

$$
(\tilde{\rho}, \tilde{u})(Y, \theta)=\frac{1}{\varepsilon^{2}}\left((R, U)\left(\frac{Y-\tilde{\sigma} \theta}{\varepsilon}\right)-(\varrho, 0)\right)=(\hat{R}, \hat{U})(Y-\tilde{\sigma} \theta)
$$

if we set

$$
(R, U)(x)=(\varrho, 0)+\varepsilon^{2}(\hat{R}, \hat{U})(\varepsilon x) .
$$

As far as $(\mathrm{EK})$ is concerned, we know by Theorem 4 that $(\tilde{\rho}, \tilde{u})$ is such that $\mathrm{w} \stackrel{\text { def }}{=}$ $\frac{1}{2}\left(\tilde{\rho}+\varrho c^{-1} \tilde{u}\right)$ approximately solves the $\mathrm{KdV}$ equation

$$
\partial_{\theta} \mathrm{w}+\Gamma \mathrm{w} \partial_{Y} \mathrm{w}=\kappa \partial_{Y}^{3} \mathrm{w} .
$$

Therefore, $W \stackrel{\text { def }}{=} \frac{1}{2}\left(\hat{R}+\varrho c^{-1} \hat{U}\right)$ is close to the profile of a travelling wave solution to this $\mathrm{KdV}$ equation with speed $\tilde{\sigma}$. This can also be seen on the profile equations themselves, which is especially interesting for (gEK), for which we do not have a result like Theorem 4 at hand.

Theorem 5 Let $\varrho>0$ be such that $\frac{\partial^{2} \mathscr{F}}{\partial \rho^{2}}(\varrho, 0)>0$ and $\frac{\partial^{2} \mathscr{F}}{\partial \rho_{x}^{2}}(\varrho, 0)>0$. We denote as before

$$
\mathfrak{c}=\sqrt{\varrho \frac{\partial^{2} \mathscr{F}}{\partial \rho^{2}}(\varrho, 0)}, \Gamma=\frac{3 \mathfrak{c}}{2 \varrho}+\frac{\varrho}{2 \mathfrak{c}} \frac{\partial^{3} \mathscr{F}}{\partial \rho^{3}}(\varrho, 0), \quad \kappa=\frac{\varrho}{2 \mathfrak{c}} \frac{\partial^{2} \mathscr{F}}{\partial \rho_{x}^{2}}(\varrho, 0),
$$


and assume that $\Gamma>0$ (which is the case in 'standard' fluids). Let $\mathrm{w}=W(Y-\tilde{\sigma} \theta)$ be a travelling wave of speed $\tilde{\sigma}>0$ solution to the $\mathrm{KdV}$ equation

$$
\partial_{\theta} \mathrm{w}+\Gamma \mathrm{w} \partial_{Y} \mathrm{w}=\kappa \partial_{Y}^{3} \mathrm{w}
$$

and more precisely such that

$$
\frac{1}{2} \kappa W^{\prime 2}-\frac{1}{6} \Gamma W^{3}+\frac{1}{2} \tilde{\sigma} W^{2}=m \in\left(0, m_{0}\right), m_{0} \stackrel{\text { def }}{=} \frac{2 \tilde{\sigma}^{3}}{3 \Gamma^{2}} .
$$

Then there exists $\varepsilon_{0}>0$ such that for all $\varepsilon \in\left(0, \varepsilon_{0}\right]$, there is a periodic traveling wave $(\rho, u)(x, t)=(R, U)(x-\sigma t)$ solution to $(\mathrm{gEK})$ with $\sigma=\mathfrak{c}+\varepsilon^{2} \tilde{\sigma}$, verifying (31) with

$$
j=-\varrho\left(\mathfrak{c}+\frac{1}{2} \varepsilon^{2} \tilde{\sigma}\right), \lambda=\frac{\partial \mathscr{F}}{\partial \rho}(\varrho, 0)+\frac{j^{2}}{2 \varrho^{2}}, \mu=\varrho \frac{\partial \mathscr{F}}{\partial \rho}(\varrho, 0)-\mathscr{F}(\varrho, 0)+\frac{j^{2}}{\varrho}+\frac{2 \mathfrak{c}}{\varrho} \varepsilon^{6} m,
$$

and

$$
(R, U)(x)=(\varrho, 0)+\varepsilon^{2}(\hat{R}, \hat{U})(\varepsilon x)
$$

with

$$
\inf _{\xi \in \mathbb{R}}\|\hat{R}(\cdot+\xi)-W\|_{W^{1, \infty}}=\mathcal{O}\left(\varepsilon^{2}\right)
$$

From a straightforward phase portrait analysis of the KdV travelling wave ODEs, using that $\tilde{\sigma}$ is positive we see that the wave profile $W$ is indeed periodic for $m \in\left(0, m_{0}\right)$, and homoclinic to $\rho_{0} \stackrel{\text { def }}{=} 2 \tilde{\sigma} / \Gamma$ in the limiting case $m=m_{0}$. As explained above, there is no hope to get a solitary wave solution to $(\mathrm{gEK})$ that is homoclinic to $\varrho$ if $j^{2}>\varrho^{2} \mathfrak{c}^{2}$. This explains why the $\mathrm{KdV}$ regime for solitary waves requires $\tilde{\sigma}<0$, so that $j=-\varrho\left(\mathfrak{c}+\frac{1}{2} \varepsilon^{2} \tilde{\sigma}\right)$ implies $j^{2}<\varrho^{2} \mathfrak{c}^{2}$ for $\varepsilon$ small enough. The KdV regime for solitary waves is a little simpler than for periodic waves, and can be justified as follows.

Theorem 6 With the same notations as in Theorem 5, we consider a travelling wave for $(\mathrm{KdV})$ of speed $\tilde{\sigma}<0, \mathrm{w}=W(Y-\tilde{\sigma} \theta)$ such that

$$
\frac{1}{2} \kappa W^{\prime 2}-\frac{1}{6} \Gamma W^{3}+\frac{1}{2} \tilde{\sigma} W^{2}=0 .
$$

Then there exists $\varepsilon_{0}>0$ such that for all $\varepsilon \in\left(0, \varepsilon_{0}\right]$, there is a solitary traveling wave $(\rho, u)(x, t)=(R, U)(x-\sigma t)$ solution to $(\mathrm{gEK})$ with $\sigma=\mathfrak{c}+\varepsilon^{2} \tilde{\sigma}$, verifying (31) with

$$
j=-\varrho\left(\mathfrak{c}+\frac{1}{2} \varepsilon^{2} \tilde{\sigma}\right), \lambda=\frac{\partial \mathscr{F}}{\partial \rho}(\varrho, 0)+\frac{j^{2}}{2 \varrho^{2}}, \quad \mu=\varrho \frac{\partial \mathscr{F}}{\partial \rho}(\varrho, 0)-\mathscr{F}(\varrho, 0)+\frac{j^{2}}{\varrho},
$$

and

$$
(R, U)(x)=(\varrho, 0)+\varepsilon^{2}(\hat{R}, \hat{U})(\varepsilon x)
$$

with

$$
\inf _{\xi \in \mathbb{R}}\|\hat{R}(Y+\xi)-W(Y)\|_{W^{1, \infty}}=\mathcal{O}\left(\varepsilon^{2}\right) .
$$

This result was already known for the KdV regime associated with (NLS), see [12]. 


\section{Approximation by counter propagating waves}

\subsection{Statement of error bounds for counter propagating waves}

For the extension of Theorem 4 to left and right-going waves, we can no longer change frame. In order to secure the interaction between those two waves, we shall assume an additional bound on the initial data, ensuring some kind of decay at infinity for the solutions of the $\mathrm{KdV}$ equations. For the similar issue concerning water waves, some control on $\left(1+X^{2}\right)^{2}\left(\left(\hat{\rho}^{\text {in }} \pm\right.\right.$ $\left.\left.\varrho \hat{u}^{\mathrm{in}} / \mathfrak{c}\right)\right)$ in $L^{2}$ was required by Schneider and Wayne [25]. Béthuel, Gravejat, Saut and Smets [8] replaced this assumption by a weaker one, expressed in terms of the $\mathcal{M}$-norm defined by

$$
\|F\|_{\mathcal{M}} \stackrel{\text { def }}{=} \sup _{a, b \in \mathbb{R}}\left|\int_{a}^{b} F\right| .
$$

Theorem 7 We assume $d=1$, and take an integer $s \geqslant 3$, and a real number $M>0$. For $\eta \in(0,1], \varepsilon \in(0,1]$, any initial data

$$
\left(\hat{\rho}^{\text {in }}, \hat{u}^{\text {in }}\right) \in \mathcal{B}_{s, \varepsilon}(M)=\left\{(\hat{\rho}, \hat{u}) \in H^{s+1}(\mathbb{R}) \times H^{s}(\mathbb{R}) ;\|(\hat{\rho}, \hat{u})\|_{\left(H^{s}(\mathbb{R})\right)^{2}}+\varepsilon\|\hat{\rho}\|_{H^{s+1}(\mathbb{R})} \leqslant M\right\}
$$

is associated with the solution $(\hat{\rho}, \hat{u}) \in \mathscr{C}\left(\left[0, T_{*} / \eta\right], \mathcal{B}_{s, \varepsilon}(2 M)\right)$ of $\left(\mathrm{EK}_{\varepsilon, \eta}\right)$ such that $(\hat{\rho}, \hat{u})(0)=$ $\left(\hat{\rho}^{\mathrm{in}}, \hat{u}^{\mathrm{in}}\right)$, as given by Theorem 2 in the case $d=1$. We also introduce $\mathfrak{Z}^{ \pm} \in \mathscr{C}\left(\left[0, \theta_{*}^{ \pm}\right], H^{s}(\mathbb{R})\right)$ solutions of the uncoupled, inviscid Burgers equations

$$
\partial_{\theta} \mathfrak{Z}^{ \pm} \pm \Gamma \mathfrak{Z}^{ \pm} \partial_{X} \mathfrak{Z}^{ \pm}=0
$$

such that $\mathfrak{Z}^{ \pm}(0)=\left(\hat{\rho}^{\text {in }} \pm \varrho \hat{u}^{\text {in }} / \mathfrak{c}\right) / 2$, where the times of existence $\theta_{*}^{ \pm}$depend continuously on $M$, and $\zeta^{ \pm} \in \mathscr{C}\left([0,+\infty), H^{s}(\mathbb{R})\right)$ the global solutions of the uncoupled $\mathrm{KdV}$ equations

$$
\partial_{\theta} \zeta^{ \pm} \pm \Gamma \zeta^{ \pm} \partial_{X} \zeta^{ \pm}= \pm \frac{\varepsilon^{2}}{\eta} \kappa \partial_{X}^{3} \zeta^{ \pm}
$$

such that $\zeta^{ \pm}(0)=\left(\hat{\rho}^{\text {in }} \pm \varrho \hat{u}^{\text {in }} / \mathfrak{c}\right) / 2$. If, in addition,

$$
\left\|\left(\hat{\rho}^{\text {in }}, \hat{u}^{\text {in }}\right)\right\|_{\mathcal{M}} \leqslant M
$$

then there exists a constant $C$, depending only on $s$ and $M$, so that for $0 \leqslant T \leqslant \min \left(T_{*}, \theta_{*}^{+}, \theta_{*}^{-}\right) / \eta$, the following hold:

$\left\|\frac{1}{2}\left(\hat{\rho}+\frac{\varrho}{\mathfrak{c}} \hat{u}\right)(T)-\mathfrak{Z}^{+}(\eta T, \cdot-\mathfrak{c} T)\right\|_{H^{s-3}}+\left\|\frac{1}{2}\left(\hat{\rho}-\frac{\varrho}{\mathfrak{c}} \hat{u}\right)(T)-\mathfrak{Z}^{-}(\eta T, \cdot+\mathfrak{c} T)\right\|_{H^{s-3}} \leqslant C\left(\eta+\frac{\varepsilon^{2}}{\eta}\right)$.

(ii). If in addition $s \geqslant 6$ and $\eta \leqslant \varepsilon^{2}$, then

$$
\left\|\frac{1}{2}\left(\hat{\rho}+\frac{\varrho}{\mathfrak{c}} \hat{u}\right)(T)-\zeta^{+}(\eta T,-\mathfrak{c} T)\right\|_{H^{s-6}}+\left\|\frac{1}{2}\left(\hat{\rho}-\frac{\varrho}{\mathfrak{c}} \hat{u}\right)(T)-\zeta^{-}(\eta T, \cdot+\mathfrak{c} T)\right\|_{H^{s-6}} \leqslant C \varepsilon^{2} .
$$


The proof in [8] relies on a careful (and lengthy) estimate of the interaction terms, such as those involving

$$
\partial_{X}\left(\frac{1}{2}\left(\hat{\rho}+\frac{\varrho}{\mathfrak{c}} \hat{u}\right) \frac{1}{2}\left(\hat{\rho}-\frac{\varrho}{\mathfrak{c}} \hat{u}\right)\right) .
$$

We use instead a systematic approach, and prove an error estimate between the solution and an approximate solution involving the two counter propagating waves, as well as terms associated with these interaction terms. This simplifies the energy estimate performed in [8], and gives a natural explanation for introducing the $\mathcal{M}$-norm. The drawback of our approach is that we lose one more derivative.

\subsection{Detailed proof of error bounds for counter propagating waves}

Construction of an approximate solution in case $(i i)\left(s \geqslant 6, \eta \leqslant \varepsilon^{2}\right)$. The aim of this paragraph is to construct an approximate solution to (19) up to a sufficiently small error, namely $\mathcal{O}\left(\eta\left(\eta+\varepsilon^{2}\right)\right)$. We consider $\zeta^{ \pm}$, solutions of the $\mathrm{KdV}$ equations in (33) associated with the initial data $\left(\hat{\rho}^{\text {in }} \pm \varrho \hat{u}^{\text {in }} / \mathfrak{c}\right) / 2$, and seek approximate solutions of the form

$$
\begin{gathered}
\hat{\rho}^{\text {app }}(T, X) \stackrel{\text { def }}{=} \zeta^{+}(\eta T, X-\mathfrak{c} T)+\zeta^{-}(\eta T, X+\mathfrak{c} T)+\eta \hat{\rho}^{1}(\eta T, T, X), \\
\hat{u}^{\text {app }}(T, X) \stackrel{\text { def }}{=} \frac{\mathfrak{c}}{\varrho}\left(\zeta^{+}(\eta T, X-\mathfrak{c} T)-\zeta^{-}(\eta T, X+\mathfrak{c} T)\right)+\eta \hat{u}^{1}(\eta T, T, X),
\end{gathered}
$$

where $\left(\hat{\rho}^{1}, \hat{u}^{1}\right)$ will be defined later but have to be thought of order one in $\eta$. Then

$$
\begin{aligned}
\operatorname{Err}_{\rho} \stackrel{\text { def }}{=} & \partial_{T} \hat{\rho}^{\text {app }}+\partial_{X}\left(\left(\varrho+\eta \hat{\rho}^{\text {app }}\right) \hat{u}^{\text {app }}\right) \\
= & \eta \partial_{\theta} \zeta^{+}(\eta T, X-\mathfrak{c} T)+\eta \partial_{\theta} \zeta^{-}(\eta T, X+\mathfrak{c} T) \\
& +\eta \frac{\mathfrak{c}}{\varrho}\left(\partial_{X}\left(\left[\zeta^{+}(\eta T, X-\mathfrak{c} T)\right]^{2}\right)-\partial_{X}\left(\left[\zeta^{-}(\eta T, X+\mathfrak{c} T)\right]^{2}\right)\right) \\
& +\eta \partial_{T} \hat{\rho}^{1}(\eta T, T, X)+\eta \varrho \partial_{X} \hat{u}^{1}(\eta T, T, X)+\eta^{2} \partial_{\theta} \hat{\rho}^{1}(\eta T, T, X) \\
& +\eta^{2} \partial_{X}\left(\hat{\rho}^{1}(\eta T, T, X) \hat{u}^{1}(\eta T, T, X)\right)
\end{aligned}
$$

and

$$
\begin{aligned}
& \operatorname{Err}_{u} \stackrel{\text { def }}{=} \partial_{T} \hat{u}^{\mathrm{app}}+\eta \hat{u}^{\mathrm{app}} \partial_{X} \hat{u}^{\mathrm{app}}+g^{\prime}\left(\varrho+\eta \hat{\rho}^{\mathrm{app}}\right) \partial_{X} \hat{\rho}^{\mathrm{app}} \\
&-\varepsilon^{2} \partial_{X}\left(K\left(\varrho+\eta \hat{\rho}^{\mathrm{app}}\right) \partial_{X}^{2} \hat{\rho}^{\mathrm{app}}+\frac{\eta}{2} K^{\prime}\left(\varrho+\eta \hat{\rho}^{\mathrm{app}}\right)\left(\partial_{X} \hat{\rho}^{\mathrm{app}}\right)^{2}\right) \\
&= \mathcal{S}+\eta \partial_{T} \hat{u}^{1}(\eta T, T, X)+\eta g^{\prime}(\varrho) \partial_{X} \hat{\rho}^{1}(\eta T, T, X)+\eta^{2} \partial_{\theta} \hat{u}^{1}(\eta T, T, X)+\mathcal{R},
\end{aligned}
$$


where $\mathcal{S}$ contains the terms of formal order $\eta$, namely

$$
\begin{aligned}
\mathcal{S} \stackrel{\text { def }}{=} & \eta \frac{\mathfrak{c}}{\varrho} \partial_{\theta} \zeta^{+}(\eta T, X-\mathfrak{c} T)-\eta \frac{\mathfrak{c}}{\varrho} \partial_{\theta} \zeta^{-}(\eta T, X+\mathfrak{c} T) \\
& +\frac{\eta \mathfrak{c}^{2}}{\varrho^{2}} \zeta^{+}(\eta T, X-\mathfrak{c} T) \partial_{X} \zeta^{+}(\eta T, X-\mathfrak{c} T)+\frac{\eta \mathfrak{c}^{2}}{\varrho^{2}} \zeta^{-}(\eta T, X+\mathfrak{c} T) \partial_{X} \zeta^{-}(\eta T, X+\mathfrak{c} T) \\
& -\frac{\eta \mathfrak{c}^{2}}{\varrho^{2}} \partial_{X}\left[\zeta^{+}(\eta T, X-\mathfrak{c} T) \zeta^{-}(\eta T, X+\mathfrak{c} T)\right] \\
& +\eta g^{\prime}(\varrho) \partial_{X} \hat{\rho}^{1}(\eta T, T, X) \\
& +g^{\prime \prime}(\varrho) \eta\left(\zeta^{+}(\eta T, X-\mathfrak{c} T) \partial_{X} \zeta^{+}(\eta T, X-\mathfrak{c} T)+\zeta^{-}(\eta T, X+\mathfrak{c} T) \partial_{X} \zeta^{-}(\eta T, X+\mathfrak{c} T)\right) \\
& +g^{\prime \prime}(\varrho) \eta \partial_{X}\left[\zeta^{+}(\eta T, X-\mathfrak{c} T) \zeta^{-}(\eta T, X+\mathfrak{c} T)\right] \\
& -\varepsilon^{2} K(\varrho) \partial_{X}^{3} \zeta^{+}(\eta T, X-\mathfrak{c} T)-\varepsilon^{2} K(\varrho) \partial_{X}^{3} \zeta^{-}(\eta T, X+\mathfrak{c} T),
\end{aligned}
$$

and $\mathcal{R}$ contains terms of formal order $\mathcal{O}\left(\eta\left(\eta+\varepsilon^{2}\right)\right)$, namely

$$
\begin{aligned}
\mathcal{R} \stackrel{\text { def }}{=} & \frac{\eta^{2} \mathfrak{c}}{2 \varrho} \partial_{X}\left[\left(\zeta^{+}(\eta T, X-\mathfrak{c} T)-\zeta^{-}(\eta T, X+\mathfrak{c} T)\right) \hat{u}^{1}(\eta T, T, X)\right] \\
& +\eta^{3} \hat{u}^{1}(\eta T, T, X) \partial_{X} \hat{u}^{1}(\eta T, T, X) \\
& +\eta\left(g^{\prime}\left(\varrho+\eta \hat{\rho}^{\mathrm{app}}\right)-g^{\prime}(\varrho)\right) \partial_{X} \hat{\rho}^{1}(\eta T, T, X) \\
& +\left(g^{\prime}\left(\varrho+\eta \hat{\rho}^{\mathrm{app}}\right)-g^{\prime}(\varrho)-g^{\prime \prime}(\varrho) \eta \hat{\rho}^{\mathrm{app}}\right) \partial_{X}\left(\zeta^{+}(\eta T, X-\mathfrak{c} T)+\zeta^{-}(\eta T, X+\mathfrak{c} T)\right) \\
& +g^{\prime \prime}(\varrho) \eta^{2} \hat{\rho}^{1}(\eta T, T, X) \partial_{X}\left(\zeta^{+}(\eta T, X-\mathfrak{c} T)+\zeta^{-}(\eta T, X+\mathfrak{c} T)\right) \\
& -\varepsilon^{2} \eta K(\varrho) \partial_{X}^{3} \hat{\rho}^{1}(\eta T, T, X) \\
& -\varepsilon^{2} \partial_{X}\left(\left[K(\varrho)-K\left(\varrho+\eta \hat{\rho}^{\mathrm{app}}\right)\right] \partial_{X}^{2} \hat{\rho}^{\mathrm{app}}\right)-\frac{\eta \varepsilon^{2}}{2} \partial_{X}\left(K^{\prime}\left(\varrho+\eta \hat{\rho}^{\mathrm{app}}\right)\left(\partial_{X} \hat{\rho}^{\mathrm{app}}\right)^{2}\right)
\end{aligned}
$$

At this stage we see the interaction terms, like $\partial_{X}\left[\zeta^{+}(\eta T, X-\mathfrak{c} T) \zeta^{-}(\eta T, X+\mathfrak{c} T)\right]$, appearing in $\mathcal{S}$. We wish to define $\left(\hat{\rho}^{1}, \hat{u}^{1}\right)$ so that the terms of formal order $\eta$ and $\varepsilon^{2}$ cancel out, that is as the solutions of

$$
\left\{\begin{array}{l}
\partial_{T} \hat{\rho}^{1}(\theta, T, X)+\varrho \partial_{X} \hat{u}^{1}(\theta, T, X) \\
=-\partial_{\theta} \zeta^{+}(\theta, X-\mathfrak{c} T)-\partial_{\theta} \zeta^{-}(\theta, X+\mathfrak{c} T) \\
\quad-\frac{\mathfrak{c}}{\varrho}\left(\partial_{X}\left(\left[\zeta^{+}(\theta, X-\mathfrak{c} T)\right]^{2}\right)-\partial_{X}\left(\left[\zeta^{-}(\theta, X+\mathfrak{c} T)\right]^{2}\right)\right), \\
\partial_{T} \hat{u}^{1}(\theta, T, X)+g^{\prime}(\varrho) \partial_{X} \hat{\rho}^{1}(\theta, T, X)=\frac{\mathcal{S}}{\eta} .
\end{array}\right.
$$

Here, $\theta$ is seen as a parameter, and for $T=0$, we set zero initial data. In addition, $\mathcal{S}$ is 
evaluated at $\theta$ instead of $\eta T$. The system above equivalently reads

$$
\begin{aligned}
& {\left[\partial_{T}+\mathfrak{c} \partial_{X}\right]\left(\hat{\rho}^{1}+\varrho \mathfrak{c}^{-1} \hat{u}^{1}\right)(\theta, T, X)=} \\
& \quad-2\left[\partial_{\theta} \zeta^{+}+\Gamma \zeta^{+} \partial_{X} \zeta^{+}-\frac{\varepsilon^{2}}{\eta} \kappa \partial_{X}^{3} \zeta^{+}\right](\theta, X-\mathfrak{c} T) \\
& \quad-\left(\frac{\varrho}{\mathfrak{c}} g^{\prime \prime}(\varrho)+\frac{\mathfrak{c}}{\varrho}\right)\left(\zeta^{-} \partial_{X} \zeta^{-}\right)(\theta, X+\mathfrak{c} T) \\
& \quad-\left(\frac{\varrho}{\mathfrak{c}} g^{\prime \prime}(\varrho)-\frac{\mathfrak{c}}{\varrho}\right) \partial_{X}\left[\zeta^{+}(\theta, X-\mathfrak{c} T) \zeta^{-}(\theta, X+\mathfrak{c} T)\right] \\
& \quad+\frac{2 \kappa \varepsilon^{2}}{\eta} \partial_{X}^{3} \zeta^{-}(\theta, X+\mathfrak{c} T)
\end{aligned}
$$

and

$$
\begin{aligned}
& {\left[\partial_{T}-\mathfrak{c} \partial_{X}\right]\left(\hat{\rho}^{1}-\varrho \mathfrak{c}^{-1} \hat{u}^{1}\right)(\theta, T, X)=} \\
& \quad-2\left[\partial_{\theta} \zeta^{-}-\Gamma \zeta^{-} \partial_{X} \zeta^{-}-\frac{\varepsilon^{2}}{\eta} \kappa \partial_{X}^{3} \zeta^{-}\right](\theta, X-\mathfrak{c} T) \\
& \quad+\left(\frac{\varrho}{\mathfrak{c}} g^{\prime \prime}(\varrho)-\frac{\mathfrak{c}}{\varrho}\right)\left(\zeta^{-} \partial_{X} \zeta^{-}\right)(\theta, X+\mathfrak{c} T) \\
& \quad+\left(\frac{\varrho}{\mathfrak{c}} g^{\prime \prime}(\varrho)-\frac{\mathfrak{c}}{\varrho}\right) \partial_{X}\left[\zeta^{+}(\theta, X-\mathfrak{c} T) \zeta^{-}(\theta, X+\mathfrak{c} T)\right] \\
& \quad-\frac{2 \kappa \varepsilon^{2}}{\eta} \partial_{X}^{3} \zeta^{+}(\theta, X+\mathfrak{c} T)
\end{aligned}
$$

Notice that the second line in (34) and (35)) vanish since $\zeta^{+}$and $\zeta^{-}$satisfy KdV equations. The next lemma will allow us to derive estimates for the solutions associated with the remaining interaction terms in the transport equation (34), which have a specific form.

Lemma 4 (i) For $F \in H^{s}(\mathbb{R})$ the solution $h$ of

$$
\left[\partial_{T}+\mathfrak{c} \partial_{X}\right] h(T, X)=\partial_{X} F(X+\mathfrak{c} T), \quad h_{\mid T=0}=0
$$

satisfies, for any $T \geqslant 0,\|h(T)\|_{H^{s}} \leqslant\|F\|_{H^{s}} / \mathfrak{c}$.

(ii) Assume that $s$ is a nonzero integer, that $F^{+}, F^{-} \in H^{s}(\mathbb{R})$ and that $\left\|F^{-}\right\|_{\mathcal{M}}<+\infty$. Then the solution $h$ of

$$
\left[\partial_{T}+\mathfrak{c} \partial_{X}\right] h(T, X)=\partial_{X}\left[F^{+}(X-\mathfrak{c} T) F^{-}(X+\mathfrak{c} T)\right], \quad h_{\mid T=0}=0
$$

satisfies

$$
\|h(T)\|_{H^{s-1}} \leqslant C\left\|F^{+}\right\|_{H^{s}}\left(\left\|F^{-}\right\|_{H^{s}}+\left\|F^{-}\right\|_{\mathcal{M}}\right)
$$

for all $T \geqslant 0$, where $C$ depends only on $s$ and $\mathfrak{c}$.

When the source term is not an exact spatial derivative, we refer to Proposition 3.2 in [20], where a norm growing like $\sqrt{t}$ is in general unavoidable. This growth is prevented by 
our assumption on the boundedness of the $\mathcal{M}$ norm of the left-going part of the right-hand side.

Proof. The estimate $(i)$ is a direct consequence of the explicit formula $h(T, X)=(F(X+$ $\mathfrak{c} T)-F(X-\mathfrak{c} T)) /(2 \mathfrak{c})$. For the proof of $(i i)$, we obtain by the method of characteristics the explicit formula

$$
\begin{aligned}
h(T, X) & =\int_{0}^{T} \partial_{X}\left[F^{+}(X-\mathfrak{c} T) F^{-}(X-\mathfrak{c} T+2 \mathfrak{c} \tau)\right] d \tau \\
& =\int_{0}^{T} \partial_{X} F^{+}(X-\mathfrak{c} T) F^{-}(X-\mathfrak{c} T+2 \mathfrak{c} \tau) d \tau+\int_{0}^{T} F^{+}(X-\mathfrak{c} T) \partial_{X} F^{-}(X-\mathfrak{c} T+2 \mathfrak{c} \tau) d \tau \\
& =\frac{1}{2 \mathfrak{c}} \partial_{X} F^{+}(X-\mathfrak{c} T) \int_{X-\mathfrak{c} T}^{X+\mathfrak{c} T} F^{-}+F^{+}(X-\mathfrak{c} T)\left[F^{-}(X+\mathfrak{c} T)-F^{-}(X-\mathfrak{c} T)\right] .
\end{aligned}
$$

Since $X \mapsto \int_{X-\mathfrak{c} T}^{X+\mathfrak{c} T} F^{-}$is bounded in $L^{\infty}$ by $\left\|F^{-}\right\|_{\mathcal{M}}$ (independently of $T$ ), we immediately conclude that

$$
\|h(T)\|_{L^{2}} \leqslant C\left\|F^{+}\right\|_{H^{1}}\left(\left\|F^{-}\right\|_{L^{2}}+\left\|F^{-}\right\|_{\mathcal{M}}\right) .
$$

The higher order estimates follow the same lines - alternatively, we may differentiate our transport equation, and observe that if $F \in H^{1}$, then $\left\|\partial_{X} F\right\|_{\mathcal{M}} \leqslant 2 C\|F\|_{H^{1}}$, by Sobolev embedding.

In order to apply Lemma 4 , we need a bound on the $\mathcal{M}$ norms of $\zeta^{ \pm}$for $0 \leqslant \theta \leqslant \theta_{*}^{ \pm}$.

Lemma 5 Let $s$ be a real number, $s \geqslant 3$, and $\zeta \in \mathscr{C}\left(\left[0, \theta_{*}\right], H^{s}\right)$ be a solution of the $\mathrm{KdV}$ equation

$$
\partial_{\theta} \zeta+\Gamma \zeta \partial_{X} \zeta=\frac{\varepsilon^{2}}{\eta} \partial_{X}^{3} \zeta
$$

Then, for any $0 \leqslant \theta \leqslant \theta_{*}$, we have

$$
\|\zeta(\theta)\|_{\mathcal{M}} \leqslant\|\zeta(0)\|_{\mathcal{M}}+C \int_{0}^{\theta}\left(\|\zeta(\omega)\|_{H^{s}}^{2}+\frac{\varepsilon^{2}}{\eta}\|\zeta(\omega)\|_{H^{s}}\right) d \omega,
$$

where the constant $C$ depends only on $\Gamma$.

Proof. We consider two real numbers $a<b$ and simply write

$$
\begin{aligned}
& \int_{a}^{b} \zeta(\theta, X) d X-\int_{a}^{b} \zeta(\theta=0, X) d X \\
& =\int_{a}^{b} \int_{0}^{\theta} \partial_{\theta} \zeta(\omega, X) d \omega d X=-\int_{0}^{\theta} \Gamma \int_{a}^{b} \zeta \partial_{X} \zeta d X d \omega+\frac{\varepsilon^{2}}{\eta} \int_{0}^{\theta} \int_{a}^{b} \partial_{X}^{3} \zeta d X d \omega \\
& =-\frac{\Gamma}{2} \int_{0}^{\theta}\left(\zeta^{2}(\omega, b)-\zeta^{2}(\omega, a)\right) d \omega+\frac{\varepsilon^{2}}{\eta} \int_{0}^{\theta}\left(\partial_{X}^{2} \zeta(\omega, b)-\partial_{X}^{2} \zeta(\omega, a)\right) d \omega .
\end{aligned}
$$

We then obtain

$$
\left|\int_{a}^{b} \zeta(\theta, X) d X\right| \leqslant\left|\int_{a}^{b} \zeta(\theta=0, X) d X\right|+C \int_{0}^{\theta}\|\zeta(\omega)\|_{L^{\infty}}^{2}+\frac{\varepsilon^{2}}{\eta}\left\|\partial_{X}^{2} \zeta(\omega)\right\|_{L^{\infty}} d \omega,
$$


and use the Sobolev embedding to conclude.

Lemma 5 implies in particular that, for $0 \leqslant \theta \leqslant \theta_{*}^{ \pm}$,

$$
\left\|\zeta^{ \pm}(\theta)\right\|_{\mathcal{M}} \leqslant C(M)
$$

provided $\varepsilon^{2} \leqslant \eta$. As already mentioned, only three terms remain in the right-hand side of (34) and (35), since $\zeta^{+}$and $\zeta^{-}$solve the $\mathrm{KdV}$ equation in (33) with the + and - sign respectively. The first and last terms fall into case $(i)$ in Lemma 4, while the second one is as in case $(i i)$. By the superposition property of transport equations, we can add the contributions of these terms given by Lemma 4. Thanks to the estimates of $\zeta^{ \pm}$resulting from Lemma 5 , this eventually yields

$$
\sup _{0 \leqslant \eta T, \theta \leqslant \min \left(T_{*}, \theta_{*}^{+}, \theta_{*}^{-}\right)}\left\|\hat{\rho}^{1}+\varrho \mathfrak{c}^{-1} \hat{u}^{1}\right\|_{H^{s-2}} \leqslant C(M)
$$

and

$$
\sup _{0 \leqslant \eta T, \theta \leqslant \min \left(T_{*}, \theta_{*}^{+}, \theta_{*}^{-}\right)}\left\|\hat{\rho}^{1}-\varrho c^{-1} \hat{u}^{1}\right\|_{H^{s-2}} \leqslant C(M)
$$

hence

$$
\sup _{0 \leqslant \eta T, \theta \leqslant \min \left(T_{*}, \theta_{*}^{+}, \theta_{*}^{-}\right)}\left\|\left(\hat{\rho}^{1}, \hat{u}^{1}\right)\right\|_{H^{s-2}} \leqslant C(M) .
$$

Consistency error and comparison estimate. To control the consistency error, we have to bound $\partial_{\theta} \hat{\rho}^{1}$ and $\partial_{\theta} \hat{u}^{1}$. Differentiating (34) with respect to $\theta$ we obtain

$$
\begin{aligned}
0= & {\left[\partial_{T}+\mathfrak{c} \partial_{X}\right] \partial_{\theta}\left(\hat{\rho}^{1}+\varrho \mathfrak{c}^{-1} \hat{u}^{1}\right)(\theta, T, X) } \\
& +\left(\frac{\varrho}{\mathfrak{c}} g^{\prime \prime}(\varrho)-\frac{\mathfrak{c}}{\varrho}\right) \partial_{X}\left(\zeta^{-} \partial_{\theta} \zeta^{-}\right)(\theta, X+\mathfrak{c} T) \\
& +\left(\frac{\varrho}{\mathfrak{c}} g^{\prime \prime}(\varrho)-\frac{\mathfrak{c}}{\varrho}\right) \partial_{X}\left[\partial_{\theta} \zeta^{+}(\theta, X-\mathfrak{c} T) \zeta^{-}(\theta, X+\mathfrak{c} T)+\zeta^{+}(\theta, X-\mathfrak{c} T) \partial_{\theta} \zeta^{-}(\theta, X+\mathfrak{c} T)\right] \\
& -\frac{2 \kappa \varepsilon^{2}}{\eta} \partial_{X}^{3} \partial_{\theta} \zeta^{-}(\theta, X+\mathfrak{c} T) .
\end{aligned}
$$

Noticing that $\partial_{\theta} \zeta^{ \pm}$is bounded in $\mathscr{C}\left(\left[0, \theta_{*}^{ \pm}\right], H^{s-3}(\mathbb{R})\right)$ by a constant depending only on $M$ (since $\varepsilon^{2} \leqslant \eta$ ) and that the $\mathcal{M}$ norm of $\partial_{\theta} \zeta^{ \pm}$is also bounded by a constant depending only on $M$ (see the proof of Lemma 5), we infer once again from Lemmas 4 and 5 that

$$
\sup _{0 \leqslant \eta T, \theta \leqslant \min \left(T_{*}, \theta_{*}^{+}, \theta_{*}^{-}\right)}\left\|\partial_{\theta}\left[\hat{\rho}^{1}+\varrho \mathfrak{c}^{-1} \hat{u}^{1}\right]\right\|_{H^{s-5}} \leqslant C(M) .
$$

Arguing similarly with $\hat{\rho}^{1}-\varrho c^{-1} \hat{u}^{1}$, we deduce

$$
\sup _{0 \leqslant \eta T, \theta \leqslant \min \left(T_{*}, \theta_{*}^{+}, \theta_{*}^{-}\right)}\left\|\left(\partial_{\theta} \hat{\rho}^{1}, \partial_{\theta} \hat{u}^{1}\right)\right\|_{H^{s-5}} \leqslant C(M) .
$$

It then follows that $\left(\hat{\rho}^{\text {app }}, \hat{u}^{\text {app }}\right)$ is an approximate solution to 19$)$ with inital datum $\left(\hat{\rho}^{\text {in }}, \hat{u}^{\text {in }}\right)$ and a consistency error

$$
\left\|\left(\operatorname{Err}_{\rho}, \operatorname{Err}_{u}\right)\right\|_{H^{s-5}} \leqslant C \eta\left(\eta+\varepsilon^{2}\right) .
$$


Proposition 6 Let $\sigma$ and $\sigma^{\prime}$ be two integers such that $\sigma \geqslant 2$ and $0 \leqslant \sigma^{\prime} \leqslant \sigma-2$. Assume that $(\tilde{\rho}, \tilde{u}) \in \mathscr{C}\left([0, \tilde{T}], H^{\sigma+1}(\mathbb{R}) \times H^{\sigma}(\mathbb{R})\right)$ solves

$$
\left\{\begin{array}{l}
\partial_{T} \tilde{\rho}+\partial_{X}((\varrho+\eta \tilde{\rho}) \tilde{u})=\operatorname{Err}_{\tilde{\rho}} \\
\partial_{T} \tilde{u}+\eta \tilde{u} \partial_{X} \tilde{u}+g^{\prime}(\varrho+\eta \tilde{\rho}) \partial_{X} \tilde{\rho}=\varepsilon^{2} \partial_{X}\left[K(\varrho+\eta \tilde{\rho}) \partial_{X}^{2} \tilde{\rho}+\frac{\eta}{2} K^{\prime}(\varrho+\eta \tilde{\rho})\left(\partial_{X} \tilde{\rho}\right)^{2}\right]+\operatorname{Err}_{\tilde{u}}
\end{array}\right.
$$

where $\left(\operatorname{Err}_{\tilde{\rho}}, \operatorname{Err}_{\tilde{u}}\right) \in L^{\infty}\left([0, \tilde{T}], H^{\sigma^{\prime}+1}(\mathbb{R}) \times H^{\sigma^{\prime}}(\mathbb{R})\right)$. Let us denote by $(\hat{\rho}, \hat{u}) \in \mathscr{C}\left([0, \hat{T}], H^{\sigma+1}(\mathbb{R}) \times\right.$ $\left.H^{\sigma}(\mathbb{R})\right)$ a solution of $\left(\mathrm{EK}_{\varepsilon, \eta}\right)$ with $(\tilde{\rho}, \tilde{u})(0)$ as initial condition. Assume that $M>0$ is such that $(\hat{\rho}, \hat{u})(T)$ and $(\tilde{\rho}, \tilde{u})(T) \in \mathcal{B}_{\sigma, \varepsilon}(2 M)$ as long as $0 \leqslant T \leqslant \min (\tilde{T}, \hat{T})$. Then, for $0 \leqslant T \leqslant \min (\tilde{T}, \hat{T})$

$$
\begin{array}{r}
\left\|\left(\tilde{\rho}-\hat{\rho}, \tilde{u}-\hat{u}, \varepsilon \partial_{X}(\tilde{\rho}-\hat{\rho})\right)\right\|_{H^{\sigma^{\prime}}} \\
\leqslant C(\sigma, r, M) \sqrt{\eta T} \mathrm{e}^{C(\sigma, r, M) \eta T}\left(\frac{1}{\eta}\left\|\left(\operatorname{Err}_{\tilde{\rho}}, \operatorname{Err}_{\tilde{u}}, \varepsilon \partial_{X} \operatorname{Err}_{\tilde{\rho}}\right)\right\|_{L^{\infty}\left([0, \min (\tilde{T}, \hat{T})], H^{\sigma^{\prime}}\right)}+\eta+\varepsilon^{2}\right) .
\end{array}
$$

Proof. The difference $(\check{\rho}, \check{u}) \stackrel{\text { def }}{=}(\hat{\rho}, \hat{u})-(\tilde{\rho}, \tilde{u})$ satisfies the system

$$
\left\{\begin{array}{l}
\partial_{T} \check{\rho}+\partial_{X}((\varrho+\eta \hat{\rho}) \check{u})+\eta \partial_{X}(\check{\rho} \hat{u})=-\operatorname{Err}_{\tilde{\rho}} \\
\partial_{T} \check{u}+g^{\prime}(\varrho) \partial_{X} \check{\rho}+\eta \hat{u} \partial_{X} \check{u}+\eta \check{u} \partial_{X} \tilde{u}+\eta g^{\prime \prime}(\varrho) \hat{\rho} \partial_{X} \check{\rho}+\eta g^{\prime \prime}(\varrho) \check{\rho} \partial_{X} \tilde{\rho} \\
\quad=\varepsilon^{2} K(\varrho) \partial_{X}^{3} \check{\rho}-\operatorname{Err}_{\tilde{u}}+\mathcal{O}_{L^{\infty}\left([0, \min (\tilde{T}, \hat{T})], H^{\sigma-2}\right)}\left(\eta\left(\varepsilon^{2}+\eta\right)\right),
\end{array}\right.
$$

with null initial condition. In the second equation, the error term contains the remainder associated with the expansion of the nonlinearity $g(\varrho+\eta \hat{\rho})$, the term $\varepsilon^{2} \eta \partial_{X}\left(K^{\prime}(\varrho+\eta \hat{\rho})\left(\partial_{X} \hat{\rho}\right)^{2}\right)$, the difference $\varepsilon^{2} \partial_{X}\left(K(\varrho+\eta \hat{\rho}) \partial_{X}^{2} \hat{\rho}\right)-\varepsilon^{2} K(\varrho) \partial_{X}^{3} \hat{\rho}$ and the corresponding terms with $\eta \tilde{\rho}$. Then, the complex vector field

$$
\check{z} \stackrel{\text { def }}{=} \check{u}+i \check{w}, \quad \check{w} \stackrel{\text { def }}{=} \varepsilon \sqrt{\frac{K(\varrho)}{\varrho+\eta \hat{\rho}}} \partial_{X} \hat{\rho}-\varepsilon \sqrt{\frac{K(\varrho)}{\varrho+\eta \tilde{\rho}}} \partial_{X} \tilde{\rho}=\varepsilon \sqrt{\frac{K(\varrho)}{\varrho+\eta \hat{\rho}}} \partial_{X} \check{\rho}+\mathcal{O}_{H^{\sigma-1}}(\varepsilon \eta)
$$

is a solution, with zero initial datum, of

$$
\begin{aligned}
\partial_{T} \check{z} & +\eta \tilde{u} \partial_{X} \check{z}+\eta \check{u} \partial_{X} \tilde{z}+i \eta\left(\partial_{X} \check{z}\right) \hat{w}+i \eta\left(\partial_{X} \tilde{z}\right) \check{w} \\
& +\frac{1}{\varepsilon} b_{\sharp}(\varrho+\eta \hat{\rho}) \check{w}+\frac{1}{\varepsilon}\left(b_{\sharp}(\varrho+\eta \hat{\rho})-b_{\sharp}(\varrho+\eta \tilde{\rho})\right) \tilde{w} \\
& +i \varepsilon \partial_{X}\left(a_{\sharp}(\varrho+\eta \hat{\rho}) \partial_{X} \check{z}\right)+i \varepsilon \partial_{X}\left(\left(a_{\sharp}(\varrho+\eta \hat{\rho})-a_{\sharp}(\varrho+\eta \tilde{\rho})\right) \partial_{X} \tilde{z}\right) \\
& =-\operatorname{Err}_{\tilde{u}}-i \varepsilon \sqrt{\frac{K(\varrho)}{\varrho+\eta \hat{\rho}}} \partial_{X} \operatorname{Err}_{\tilde{\rho}},
\end{aligned}
$$

where

$$
a_{\sharp}(\rho) \stackrel{\text { def }}{=} \sqrt{K(\varrho) \rho}, \quad b_{\sharp}(\rho) \stackrel{\text { def }}{=} \frac{\rho\left(g^{\prime}(\varrho)+(\rho-\varrho) g^{\prime \prime}(\varrho)\right)}{a_{\sharp}(\rho)} .
$$


The terms $\eta \check{u} \partial_{X} \tilde{z}$ and $i \eta\left(\partial_{X} \tilde{z}\right) \check{w}$ are easily estimated in $H^{\sigma^{\prime}}$, using A.2, by

$$
C(\sigma) \eta\|\check{z}\|_{H^{\sigma^{\prime}}}\left\|\partial_{X} \tilde{z}\right\|_{W^{\sigma^{\prime}, \infty}} \leqslant C(\sigma, M) \eta\|\check{z}\|_{H^{\sigma^{\prime}}}\|\tilde{z}\|_{H^{\sigma}} \leqslant C(\sigma, M) \eta\|\check{z}\|_{H^{\sigma^{\prime}}}
$$

since $\sigma^{\prime}+2 \leqslant \sigma$ by assumption and by Sobolev embedding. We write similarly that the $H^{\sigma^{\prime}}$ norm of the term $\varepsilon^{-1}\left(b_{\sharp}(\varrho+\eta \hat{\rho})-b_{\sharp}(\varrho+\eta \tilde{\rho})\right) \hat{w}$ is

$$
\leqslant C(\sigma, r) \eta \varepsilon^{-1}\|\check{\rho}\|_{H^{\sigma^{\prime}}}\|\tilde{w}\|_{W^{\sigma^{\prime}, \infty}} \leqslant C(\sigma, r, M) \eta\|\check{\rho}\|_{H^{\sigma^{\prime}}}\left\|\partial_{X} \tilde{\rho}\right\|_{H^{\sigma}} \leqslant C(\sigma, r, M) \eta\|\check{\rho}\|_{H^{\sigma^{\prime}}} .
$$

By A.3 and (A.1), since $\sigma^{\prime}+2 \leqslant \sigma$,

$$
\begin{aligned}
& \left\|i \varepsilon \partial_{X}\left(\left(a_{\sharp}(\varrho+\eta \hat{\rho})-a_{\sharp}(\varrho+\eta \tilde{\rho})\right) \partial_{X} \tilde{z}\right)\right\|_{H^{\sigma^{\prime}}} \\
& \leqslant C(\sigma, r) \varepsilon\left\|\left(a_{\sharp}(\varrho+\eta \hat{\rho})-a_{\sharp}(\varrho+\eta \tilde{\rho})\right) \partial_{X} \tilde{z}\right\|_{H^{\sigma^{\prime}+1}} \\
& \leqslant C(\sigma, r) \varepsilon \eta\|\check{\rho}\|_{H^{\sigma^{\prime}+1}}\left\|\partial_{X} \tilde{z}\right\|_{H^{\sigma^{\prime}+1}} \leqslant C(\sigma, r, M) \eta\left\|\left(\check{\rho}, \varepsilon \partial_{X} \check{\rho}\right)\right\|_{H^{\sigma^{\prime}}} .
\end{aligned}
$$

Therefore, $\check{z}$ is a solution of

$$
\partial_{T} \check{z}+\eta \hat{u} \partial_{X} \check{z}+i \eta\left(\partial_{X} \check{z}\right) \hat{w}+\frac{1}{\varepsilon} b_{\sharp}(\varrho+\eta \hat{\rho}) \check{w}+i \varepsilon \partial_{X}\left(a_{\sharp}(\varrho+\eta \hat{\rho}) \partial_{X} \check{z}\right)=\mathcal{G},
$$

with zero initial datum and where, for $0 \leqslant T \leqslant \min (\tilde{T}, \hat{T})$,

$$
\|\mathcal{G}(T)\|_{H^{\sigma^{\prime}}} \leqslant C(\sigma, r, M)\left(\left\|\left(\operatorname{Err}_{\tilde{u}}, \varepsilon \partial_{X} \operatorname{Err}_{\tilde{\rho}}\right)\right\|_{H^{\sigma^{\prime}}}+\eta\left(\eta+\varepsilon^{2}\right)+\eta\left\|\left(\check{\rho}, \varepsilon \partial_{X} \check{\rho}\right)\right\|_{H^{\sigma^{\prime}}}\right) .
$$

Letting

$$
E_{\sigma^{\prime}}^{\sharp}(\check{\rho}, \check{z}) \stackrel{\text { def }}{=} \sum_{k=0}^{\sigma^{\prime}} \dot{E}_{k}^{\sharp}(\check{\rho}, \check{z})
$$

where

$$
\dot{E}_{k}^{\sharp}(\check{\rho}, \check{z}) \stackrel{\text { def }}{=} \int_{\mathbb{R}} \frac{1}{2} a_{\sharp}^{k}(\varrho+\eta \hat{\rho})\left((\varrho+\eta \hat{\rho})\left|\partial_{X}^{k} \check{z}\right|^{2}+\left(g^{\prime}(\varrho)+g^{\prime \prime}(\varrho) \eta \hat{\rho}\right)\left(\partial_{X}^{k} \check{\rho}\right)^{2}\right) d X,
$$

and arguing as in the proof of Proposition 4, we arrive at

$$
\begin{aligned}
\frac{d}{d T} E_{\sigma^{\prime}}^{\sharp}(\check{\rho}, \check{z}) \leqslant & C(\sigma, r, M) \eta E_{\sigma^{\prime}}^{\sharp}(\check{\rho}, \check{z}) \\
& +C(\sigma, r, M) \sqrt{E_{\sigma^{\prime}}^{\sharp}(\check{\rho}, \check{z})}\left(\left\|\left(\operatorname{Err}_{\tilde{\rho}}, \operatorname{Err}_{\tilde{u}}, \varepsilon \partial_{X} \operatorname{Err}_{\tilde{\rho}}\right)\right\|_{H^{\sigma^{\prime}}}+\eta\left(\eta+\varepsilon^{2}\right)+\eta \sqrt{E_{\sigma^{\prime}}^{\sharp}(\check{\rho}, \check{z})}\right) \\
\leqslant & C(\sigma, r, M) \eta E_{\sigma^{\prime}}^{\sharp}(\check{\rho}, \check{z})+C(\sigma, r, M)\left(\frac{1}{\eta}\left\|\left(\operatorname{Err}_{\tilde{\rho}}, \operatorname{Err}_{\tilde{u}}, \varepsilon \partial_{X} \operatorname{Err}_{\tilde{\rho}}\right)\right\|_{H^{\sigma^{\prime}}}^{2}+\eta\left(\eta+\varepsilon^{2}\right)^{2}\right)
\end{aligned}
$$

for $0 \leqslant T \leqslant \min (\tilde{T}, \hat{T})$, since $\partial_{X} \hat{z}$ and $\partial_{X} \hat{\rho}$ are uniformly bounded in $L^{\infty}(\sigma>3 / 2)$. Indeed, there is only one place where we have to pay attention to the extra terms in the first equation in (36), namely when we compute

$$
\frac{d}{d T} \int_{\mathbb{R}} \frac{1}{2}\left[g^{\prime}(\varrho)+\eta \hat{\rho} g^{\prime \prime}(\varrho)\right] a_{\sharp}^{k}(\varrho+\eta \hat{\rho})\left(\partial^{k} \check{\rho}\right)^{2} d X
$$


and handle the term $\int_{\mathbb{R}} \frac{1}{2}\left[g^{\prime}(\varrho)+\eta \hat{\rho} g^{\prime \prime}(\varrho)\right] a_{\sharp}^{k}(\varrho+\eta \hat{\rho}) \partial_{T}\left[\left(\partial^{k} \check{\rho}\right)^{2}\right] d X$, which produces the extra term

$$
\int_{\mathbb{R}}\left[g^{\prime}(\varrho)+\eta \hat{\rho} g^{\prime \prime}(\varrho)\right] a_{\sharp}^{k}(\varrho+\eta \hat{\rho}) \partial^{k} \hat{\rho} \partial^{k} \operatorname{Err}_{\tilde{\rho}} d X \leqslant C(\sigma, r, M) \sqrt{E_{\sigma^{\prime}}^{\sharp}(\check{\rho}, \check{z})}\left\|\operatorname{Err}_{\tilde{\rho}}\right\|_{H^{\sigma^{\prime}}} .
$$

We complete the proof of Proposition 6 by a Gronwall-type argument.

To complete the proof of Theorem 7 ( $(i i)$, we choose $\sigma=s \geqslant 2$ and $\sigma^{\prime}=s-6 \in[0, \sigma-2]$ in order to apply Proposition 6 (notice that the term $\varepsilon \partial_{X} \operatorname{Err}_{\tilde{\rho}}$ induces the loss of one more derivative). Therefore, for $0 \leqslant T \leqslant \min \left(T_{*}, \theta_{*}^{+}, \theta_{*}^{-}\right) / \eta$,

$$
\left\|\left(\hat{\rho}^{\text {app }}-\hat{\rho}, \hat{u}^{\text {app }}-\hat{u}, \varepsilon \partial_{X}\left(\hat{\rho}^{\text {app }}-\hat{\rho}\right)\right)\right\|_{H^{s-6}} \leqslant C(\sigma, r, M)\left(\eta+\varepsilon^{2}\right),
$$

and this concludes the proof in case $(i i)$.

Proof of Theorem 7 in case $(i)$. We argue in a similar way and look for an approximate solution under the form

$$
\hat{\rho}^{\text {app }}(T, X) \stackrel{\text { def }}{=} \mathfrak{Z}^{+}(\eta T, X-\mathfrak{c} T)+\mathfrak{Z}^{-}(\eta T, X+\mathfrak{c} T)+\eta \hat{\rho}^{1}(\eta T, T, X)
$$

and

$$
\hat{u}^{\text {app }}(T, X) \stackrel{\text { def }}{=} \frac{\mathfrak{c}}{\varrho}\left(\mathfrak{Z}^{+}(\eta T, X-\mathfrak{c} T)-\mathfrak{Z}^{-}(\eta T, X+\mathfrak{c} T)\right)+\eta \hat{u}^{1}(\eta T, T, X) .
$$

Now, we include the dispersive terms in $\mathcal{R}$ instead of $\mathcal{S}$, since we want to prove a comparison result with Burgers equations. We thus define $\left(\hat{\rho}^{1}, \hat{u}^{1}\right)$ as the solutions of

$$
\begin{aligned}
0= & {\left[\partial_{T}+\mathfrak{c} \partial_{X}\right]\left(\hat{\rho}^{1}+\varrho \mathfrak{c}^{-1} \hat{u}^{1}\right)(\theta, T, X) } \\
& +2\left[\partial_{\theta} \mathfrak{Z}^{+}+\Gamma \mathfrak{Z}^{+} \partial_{X} \mathfrak{Z}^{+}\right](\theta, X-\mathfrak{c} T) \\
& +\left(\frac{\varrho}{\mathfrak{c}} g^{\prime \prime}(\varrho)-\frac{\mathfrak{c}}{\varrho}\right)\left(\mathfrak{Z}^{-} \partial_{X} \mathfrak{Z}^{-}\right)(\theta, X+\mathfrak{c} T) \\
& +\left(\frac{\varrho}{\mathfrak{c}} g^{\prime \prime}(\varrho)-\frac{\mathfrak{c}}{\varrho}\right) \partial_{X}\left[\mathfrak{Z}^{+}(\theta, X-\mathfrak{c} T) \mathfrak{Z}^{-}(\theta, X+\mathfrak{c} T)\right]
\end{aligned}
$$

and

$$
\begin{aligned}
0= & {\left[\partial_{T}-\mathfrak{c} \partial_{X}\right]\left(\hat{\rho}^{1}-\varrho \mathfrak{c}^{-1} \hat{u}^{1}\right)(\theta, T, X) } \\
& +2\left[\partial_{\theta} \mathfrak{Z}^{-}-\Gamma \mathfrak{Z}^{-} \partial_{X} \mathfrak{Z}^{-}\right](\theta, X-\mathfrak{c} T) \\
& -\left(\frac{\varrho}{\mathfrak{c}} g^{\prime \prime}(\varrho)-\frac{\mathfrak{c}}{\varrho}\right)\left(\mathfrak{Z}^{-} \partial_{X} \mathfrak{Z}^{-}\right)(\theta, X+\mathfrak{c} T) \\
& -\left(\frac{\varrho}{\mathfrak{c}} g^{\prime \prime}(\varrho)-\frac{\mathfrak{c}}{\varrho}\right) \partial_{X}\left[\mathfrak{Z}^{+}(\theta, X-\mathfrak{c} T) \mathfrak{Z}^{-}(\theta, X+\mathfrak{c} T)\right] .
\end{aligned}
$$

It follows from the above arguments that

$$
\sup _{0 \leqslant \eta T, \theta \leqslant \min \left(T_{*}, \theta_{*}^{+}, \theta_{*}^{-}\right)}\left\|\left(\hat{\rho}^{1}, \hat{u}^{1}\right)\right\|_{H^{s-1}} \leqslant C(M)
$$


and

$$
\sup _{0 \leqslant \eta T, \theta \leqslant \min \left(T_{*}, \theta_{*}^{+}, \theta_{*}^{-}\right)}\left\|\left(\partial_{\theta} \hat{\rho}^{1}, \partial_{\theta} \hat{u}^{1}\right)\right\|_{H^{s-2}} \leqslant C(M) .
$$

This implies the following estimate for the consistency error

$$
\left\|\left(\operatorname{Err}_{\rho}, \operatorname{Err}_{u}\right)\right\|_{H^{s-2}} \leqslant C\left(\eta^{2}+\varepsilon^{2}\right) .
$$

Using Proposition 6 with $\sigma=s \geqslant 2$ and $\sigma^{\prime}=s-3 \in[0, s-2]$, this finishes the proof of Theorem 7] $(i)$.

\section{The KP-I asymptotic regime}

In one space dimension, we have obtained as asymptotic equations a single $\mathrm{KdV}$ equation for well-prepared initial data, and two decoupled KdV equations for more general initial data. In higher dimensions, if one considers a weakly transverse perturbation, we expect to obtain Kadomtsev-Petviashvili (KP-I) type equations

$$
\partial_{\theta} \zeta+\Gamma \zeta \partial_{z_{1}} \zeta=\kappa \frac{\varepsilon^{2}}{\eta} \partial_{z_{1}}^{3} \zeta-\frac{\mathfrak{c}}{2} \frac{\delta^{2}}{\eta} \Delta_{z_{\perp}} \partial_{z_{1}}^{-1} \zeta .
$$

Throughout this section, we shall assume that the vector field $\mathbf{u}$ is curl-free, which is a natural hypothesis for the KP-I asymptotic regime.

\subsection{Main results}

We replace the long wave ansatz (2) by the weakly transverse long wave ansatz

$$
\rho(t, x)=\varrho+\eta \hat{\rho}(T, z) \quad \mathbf{u}(t, x)=\eta\left(\hat{\mathbf{u}}_{1}, \delta \hat{\mathbf{u}}_{\perp}\right)(T, z), \quad T=\varepsilon t, \quad z=\left(\varepsilon x_{1}, \varepsilon \delta x_{\perp}\right),
$$

where $\delta$ is another small parameter (we have changed $X$ to $z$ to keep in mind that the scaling is now weakly transverse). Usually, we take $\eta=\varepsilon^{2}=\delta^{2}$ to derive (KP-I) but we may consider weakly dispersive KP-Iequations similar to weakly dispersive KdV equation we have already obtained. Then, the Euler-Korteweg system (EK) becomes

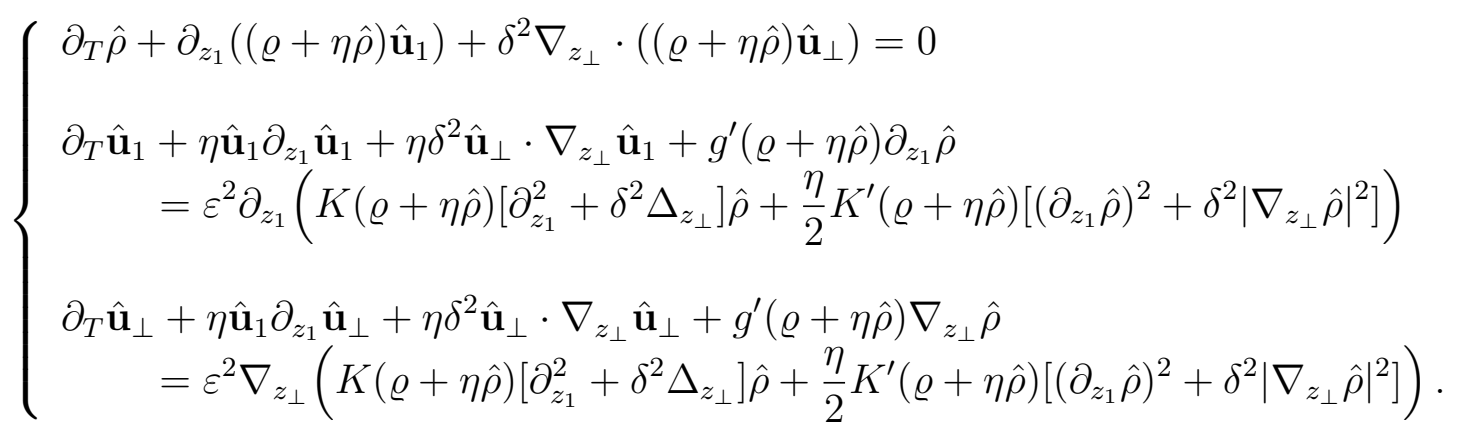


We first state a result providing uniform bounds on the time scale $T \approx \eta^{-1}$ (that is $t \approx \varepsilon^{-1} \eta^{-1}$ ) and need to define, for $s \geqslant 0$ and $M>0$, the set

$$
\begin{gathered}
\tilde{\mathcal{B}}_{s, \varepsilon, \delta}(M) \stackrel{\text { def }}{=}\left\{(\hat{\rho}, \hat{\mathbf{u}}) \in H^{s+1}\left(\mathbb{R}^{d}\right) \times\left(H^{s}\left(\mathbb{R}^{d}\right)\right)^{d} ;\right. \\
\left.\left\|\left(\hat{\rho}, \hat{\mathbf{u}}, \varepsilon \partial_{1} \hat{\rho}, \varepsilon \delta \nabla_{\perp} \hat{\rho}\right)\right\|_{H^{s}\left(\mathbb{R}^{d}\right) \times\left(H^{s}\left(\mathbb{R}^{d}\right)\right)^{d} \times H^{s}\left(\mathbb{R}^{d}\right) \times\left(H^{s}\left(\mathbb{R}^{d}\right)\right)^{d-1}} \leqslant M\right\} .
\end{gathered}
$$

Theorem 8 Let $s$ be an integer greater than $1+d / 2$ and $\eta \in(0,1]$. If $\varrho>0, g^{\prime}(\varrho)>0$, and $\left(\hat{\rho}^{\text {in }}, \hat{\mathbf{u}}^{\text {in }}\right) \in \tilde{\mathcal{B}}_{s, \varepsilon, \delta}(M)$, then there exists $T_{*}>0$, depending only on $M, s$ and $d$, such that the maximal solution to (41) such that $(\hat{\rho}, \hat{\mathbf{u}})(0)=\left(\hat{\rho}^{\mathrm{in}}, \hat{\mathbf{u}}^{\mathrm{in}}\right)$ exists at least on $\left[0, T_{*} / \eta\right]$, and $(\hat{\rho}, \hat{\mathbf{u}})(T) \in \tilde{\mathcal{B}}_{s, \varepsilon, \delta}(2 M)$ for all $T \in\left[0, T_{*} / \eta\right]$.

In this asymptotic regime, one might expect an approximation by the two counter propagating waves described by the two uncoupled KP-I equations

$$
\left\{\begin{array}{l}
\partial_{\theta} \zeta^{+}+\Gamma \zeta^{+} \partial_{z_{1}} \zeta^{+}=\frac{\varepsilon^{2}}{\eta} \kappa \partial_{z_{1}}^{3} \zeta^{+}-\frac{\mathfrak{c}}{2} \cdot \frac{\delta^{2}}{\eta} \Delta_{z_{\perp}} \partial_{z_{1}}^{-1} \zeta^{+} \\
\partial_{\theta} \zeta^{-}-\Gamma \zeta^{-} \partial_{z_{1}} \zeta^{-}=-\frac{\varepsilon^{2}}{\eta} \kappa \partial_{z_{1}}^{3} \zeta^{-}+\frac{\mathfrak{c}}{2} \cdot \frac{\delta^{2}}{\eta} \Delta_{z_{\perp}} \partial_{z_{1}}^{-1} \zeta^{-}
\end{array}\right.
$$

instead of the two KdV equations. However, Lannes has shown in [19] that, in the case $\eta=\varepsilon^{2}=\delta^{2}$ to fix ideas, the natural $\mathcal{O}\left(\varepsilon^{2}\right)$ error estimate does not hold. This is due to the singularity of the symbol associated with the operator $\Delta_{z_{\perp}} \partial_{z_{1}}^{-1}$, unless we impose the zero mass assumption $\int_{\mathbb{R}} A\left(z_{1}, z_{\perp}\right) d z_{1}=0$ for every $z_{\perp} \in \mathbb{R}^{d-1}$, which is not physical. This is the reason why Lannes and Saut have proposed in [21] weakly transverse Boussinesq type systems for which we can prove the natural error estimate and for which no zero mass assumption is made. This weakly transverse Boussinesq type system is formally equivalent to the system of two uncoupled KP-I equations (42), and it can be shown to converge to (42) (without optimal error estimates) under extra regularity and zero mass type hypothesis. In our context, a natural weakly transverse Boussinesq type system is the following:

$\left(\mathrm{B}_{\varepsilon, \delta, \eta}\right)$

$$
\left\{\begin{array}{l}
\partial_{T} \hat{\rho}+\varrho \partial_{z_{1}} \hat{\mathbf{u}}_{1}+\eta \partial_{z_{1}}\left(\hat{\rho} \hat{\mathbf{u}}_{1}\right)+\delta^{2} \nabla_{z_{\perp}} \cdot\left((\varrho+\eta \hat{\rho}) \hat{\mathbf{u}}_{\perp}\right)=0 \\
\partial_{T} \hat{\mathbf{u}}_{1}+g^{\prime}(\varrho) \partial_{z_{1}} \hat{\rho}+\eta \hat{\mathbf{u}}_{1} \partial_{z_{1}} \hat{\mathbf{u}}_{1}+\eta \delta^{2} \hat{\mathbf{u}}_{\perp} \cdot \nabla_{z_{\perp}} \hat{\mathbf{u}}_{1}+\eta \hat{\rho} g^{\prime \prime}(\varrho) \partial_{z_{1}} \hat{\rho} \\
=\varepsilon^{2} K(\varrho) \partial_{z_{1}}\left[\partial_{z_{1}}^{2}+\delta^{2} \Delta_{z_{\perp}}\right] \hat{\rho} \\
\partial_{T} \hat{\mathbf{u}}_{\perp}+g^{\prime}(\varrho) \nabla_{z_{\perp}} \hat{\rho}+\eta \hat{\mathbf{u}}_{1} \partial_{z_{1}} \hat{\mathbf{u}}_{\perp}+\eta \delta^{2} \hat{\mathbf{u}}_{\perp} \cdot \nabla_{z_{\perp}} \hat{\mathbf{u}}_{\perp}+\eta \hat{\rho} g^{\prime \prime}(\varrho) \nabla_{z_{\perp}} \hat{\rho} \\
=\varepsilon^{2} K(\varrho) \nabla_{z_{\perp}}\left[\partial_{z_{1}}^{2}+\delta^{2} \Delta_{z_{\perp}}\right] \hat{\rho} .
\end{array}\right.
$$

Let us observe that system $\left(\mathrm{B}_{\varepsilon, \delta, \eta}\right)$ may be seen as a particular case of system (41) when $g$ is a quadratic polynomial and the capillarity $K$ has constant value $K(\varrho)$. The weakly transverse Boussinesq system $\left(\mathrm{B}_{\varepsilon, \delta, \eta}\right)$ may also be seen as the weakly transverse analogue to the systems of the $(a, b, c, d)$ class introduced in [9] and [10] when $a=b=d=0$ and $c<0$. 
Theorem 9 Let $s$ be an integer such that $s>1+d / 2$ and $\eta \in(0,1]$.

(i) If $\varrho>0, g^{\prime}(\varrho)>0$, and $\left(\hat{\rho}^{\text {in }}, \hat{\mathbf{u}}^{\mathrm{in}}\right) \in \tilde{\mathcal{B}}_{s, \varepsilon, \delta}(M)$, then there exists $T_{*}>0$, depending only on $M, s$ and $d$, such that the system $\left(\mathrm{B}_{\varepsilon, \delta, \eta}\right)$ with initial datum $\left(\hat{\rho}^{\mathrm{in}}, \hat{\mathbf{u}}^{\mathrm{in}}\right)$ has a unique solution $(\hat{\rho}, \hat{\mathbf{u}}) \in \mathscr{C}\left(\left[0, T_{*} / \eta\right], H^{s+1}\left(\mathbb{R}^{d}\right) \times\left(H^{s}\left(\mathbb{R}^{d}\right)\right)^{d}\right)$. Moreover, we have $(\hat{\rho}, \hat{\mathbf{u}})(T) \in \tilde{\mathcal{B}}_{s, \varepsilon, \delta}(2 M)$ for all $T \in\left[0, T_{*} / \eta\right]$.

(ii) Assume that $\zeta^{ \pm \text {,in }}$ belongs to $\partial_{z_{1}} H^{s+7}\left(\mathbb{R}^{d}\right)$ and that $\Delta_{z_{\perp}} \zeta^{ \pm, \text {in }} \in \partial_{z_{1}}^{2} H^{s+3}\left(\mathbb{R}^{d}\right)$. Then, there exists $\theta_{*}>0$, depending only on $s, d$ and the initial data $\zeta^{ \pm \text {in }}$ such that the uncoupled system (42) has a unique solution $\zeta^{ \pm} \in \mathscr{C}\left(\left[0, \theta_{*}\right], H^{s+6}\left(\mathbb{R}^{d}\right)\right) \cap \operatorname{Lip}\left(\left[0, \theta_{*}\right], H^{s+3}\left(\mathbb{R}^{d}\right)\right)$. Moreover, one has $\zeta^{ \pm} \in L^{\infty}\left(\left[0, \theta_{*}\right], \partial_{z_{1}} H^{s+6}\left(\mathbb{R}^{d}\right)\right)$. Let us also assume that

$$
\frac{1}{2}\left(\hat{\rho}^{\text {in }}+\frac{\varrho}{\mathfrak{c}} \hat{\mathbf{u}}_{1}^{\text {in }}\right)=\zeta^{+, \text {in }}, \quad \frac{1}{2}\left(\hat{\rho}^{\text {in }}-\frac{\varrho}{\mathfrak{c}} \hat{\mathbf{u}}_{1}^{\text {in }}\right)=\zeta^{-, \text {in }}, \quad \frac{\varrho}{\mathfrak{c}} \hat{\mathbf{u}}_{\perp}^{\text {in }}=\nabla_{z_{\perp}} \partial_{z_{1}}^{-1}\left(\zeta^{+, \text {in }}-\zeta^{-, \text {in }}\right)
$$

and that

$$
\delta^{2} \leqslant \eta \quad \text { and } \quad \varepsilon^{2} \leqslant \eta .
$$

Then, the following comparison estimate with the uncoupled system (42) holds as $\eta \rightarrow 0$ :

$$
\sup _{0 \leqslant T \leqslant \min \left(\theta_{*}, T_{*}\right) / \eta}\left\|\frac{1}{2}\left(\hat{\rho}+\frac{\varrho}{\mathfrak{c}} \hat{\mathbf{u}}_{1}\right)(T)-\zeta^{+}(\eta T, \cdot-\mathfrak{c} T)\right\|_{H^{s-1}\left(\mathbb{R}^{d}\right)} \rightarrow 0
$$

and

$$
\sup _{0 \leqslant T \leqslant \min \left(\theta_{*}, T_{*}\right) / \eta}\left\|\frac{1}{2}\left(\hat{\rho}-\frac{\varrho}{\mathfrak{c}} \hat{\mathbf{u}}_{1}\right)(T)-\zeta^{-}(\eta T, \cdot+\mathfrak{c} T)\right\|_{H^{s-1}\left(\mathbb{R}^{d}\right)} \rightarrow 0 .
$$

Remark 3 The properties of the solution $\zeta^{ \pm}$to the KP-Iequation given in $(i i)$ come from [27] and [23] and use (44). The compatibility condition (43) on $\hat{\mathbf{u}}_{\perp}^{\text {in }}$ is natural since the vector field $\hat{\mathbf{u}}$ is curl-free.

Remark 4 Statement $(i)$ is a consequence of Theorem 8 in the particular case where $g$ is quadratic and $K \equiv K(\varrho)$ is constant. An alternate approach would be to use the result given in Theorem 1.1 of [24] with $a=b=d=0>c$. However, this result is stated in the Boussinesq scaling and not the weakly transverse one. It is plausible that the method of Saut and $\mathrm{Xu}$ extends to the weakly transverse case, but we have not checked this fact.

Remark 5 The proof of Theorem 9 consists in constructing an approximate solution and then proving that the error remains small. The first point requires to be able to compare $\varepsilon$, $\delta$ and $\eta$, which is the reason for assuming (44).

Remark 6 In [11, we have proposed (in the case $\eta=\varepsilon^{2}=\delta^{2}$ ) another weakly transverse Boussinesq system adapted to the case where one wave, say the left-going one, is negligible. This system has the structure of a symmetrizable hyperbolic system plus a constant coefficient skew adjoint term - not affected by the symmetrization, which is simpler than $\left(\mathrm{B}_{\varepsilon, \delta, \eta}\right)$. One may think that the dispersive terms $\varepsilon^{2} \delta^{2} \Delta_{z_{\perp}} \partial_{z_{1}} \hat{\rho}$ and $\varepsilon^{2} \delta^{2} \Delta_{z_{\perp}} \nabla_{z_{\perp}} \hat{\rho}$ in the last two equations in $\left(\mathrm{B}_{\varepsilon, \delta, \eta}\right)$ should be removable in view of their formal order $\mathcal{O}\left(\eta^{2}\right)$ behavior (by (44)). 
However, our existence and uniqueness result relies on a nonlinear symmetrization type argument which breaks down without these terms. Moreover, our estimates provide a uniform control on $\hat{\rho}, \varepsilon \partial_{1} \hat{\rho}$ and $\varepsilon \delta \nabla_{z_{\perp}} \hat{\rho}$ in $H^{s}$, so that the high order derivatives of $\varepsilon^{2} \delta^{2} \Delta_{z_{\perp}} \partial_{z_{1}} \hat{\rho}$ and $\varepsilon^{2} \delta^{2} \Delta_{z_{\perp}} \nabla_{z_{\perp}} \hat{\rho}$ are not that small.

Our last result gives a quantitative comparison estimate between system (41) and the weakly transverse system $\left(\mathrm{B}_{\varepsilon, \delta, \eta}\right)$.

Theorem 10 Let $s>2+d / 2$ (s integer), $\eta, \varepsilon, \delta \in(0,1]$ and assume that $\varrho>0, g^{\prime}(\varrho)>0$, and let $\left(\hat{\rho}^{\text {in }}, \hat{\mathbf{u}}^{\text {in }}\right) \in \tilde{\mathcal{B}}_{s, \varepsilon, \delta}(M)$. Then, there exists $T_{*}>0$, depending only on $M, s$ and $d$, such that the two systems (41), resp. $\left(\mathrm{B}_{\varepsilon, \delta, \eta}\right)$, with initial datum $\left(\hat{\rho}^{\text {in }}, \hat{\mathbf{u}}^{\text {in }}\right)$ have a unique solution $(\hat{\rho}, \hat{\mathbf{u}})$, resp. $(\tilde{\rho}, \tilde{\mathbf{u}})$, in $\mathscr{C}\left(\left[0, T_{*} / \eta\right], H^{s+1}\left(\mathbb{R}^{d}\right) \times\left(H^{s}\left(\mathbb{R}^{d}\right)\right)^{d}\right)$. Moreover, for any $T \in\left[0, T_{*} / \eta\right]$, $(\hat{\rho}, \hat{\mathbf{u}})$ and $(\tilde{\rho}, \tilde{\mathbf{u}})$ belong to $\tilde{\mathcal{B}}_{s, \varepsilon, \delta}(2 M)$. Then, there exists a constant $C$, depending only on $s$, $d$ and $M$, such that, for $0 \leqslant T \leqslant T_{*} / \eta$, we have

$$
\left\|\left(\hat{\rho}, \hat{\mathbf{u}}_{1}, \delta \hat{\mathbf{u}}_{\perp}\right)-\left(\tilde{\rho}, \tilde{\mathbf{u}}_{1}, \delta \tilde{\mathbf{u}}_{\perp}\right)\right\|_{H^{s-2}\left(\mathbb{R}^{d}\right)} \leqslant C(\eta+\varepsilon),
$$

and, for $s>3+d / 2$,

$$
\left\|\left(\hat{\rho}, \hat{\mathbf{u}}_{1}, \delta \hat{\mathbf{u}}_{\perp}\right)-\left(\tilde{\rho}, \tilde{\mathbf{u}}_{1}, \delta \tilde{\mathbf{u}}_{\perp}\right)\right\|_{H^{s-3}\left(\mathbb{R}^{d}\right)} \leqslant C\left(\eta+\varepsilon^{2}\right) .
$$

\subsection{Uniform bounds in the weakly transverse scaling}

Proof of Theorem 8. The complex vector field $\hat{\mathbf{z}}$ is now

$$
\hat{\mathbf{z}}=\left(\hat{\mathbf{z}}_{1}, \delta \hat{\mathbf{z}}_{\perp}\right)=\hat{\mathbf{u}}+i \hat{\mathbf{w}}=\left(\hat{\mathbf{u}}_{1}, \delta \hat{\mathbf{u}}_{\perp}\right)+i \varepsilon \sqrt{\frac{K(\rho)}{\rho}}\left(\partial_{1} \hat{\rho}, \delta \nabla_{\perp} \hat{\rho}\right),
$$

and the assumption that the vector field $\hat{\mathbf{u}}$ is curl free now reads

$$
\partial_{1} \hat{\mathbf{u}}_{\perp}=\nabla_{\perp} \hat{\mathbf{u}}_{1}
$$

The $L^{2}$-type functional $E_{0}$ becomes

$$
E_{0}^{\natural}[\hat{\rho}, \hat{\mathbf{z}}] \stackrel{\text { def }}{=} \frac{1}{2} \int_{\mathbb{R}^{d}} \rho\left|\hat{\mathbf{z}}_{1}\right|^{2}+\delta^{2} \rho\left|\hat{\mathbf{z}}_{\perp}\right|^{2}+g^{\prime}(\rho) \hat{\rho}^{2} d z, \quad \rho=\varrho+\eta \hat{\rho},
$$

and the $H^{s}$-type functional $E_{s}$ becomes

$$
E_{s}^{\natural}[\hat{\rho}, \hat{\mathbf{z}}] \stackrel{\text { def }}{=} \sum_{\sigma=0}^{s} \dot{E}_{\sigma}^{\natural}(\hat{\rho}, \hat{\mathbf{z}}),
$$

where we have denoted

$$
\dot{E}_{\sigma}^{\natural}[\hat{\rho}, \hat{\mathbf{z}}] \stackrel{\text { def }}{=} \sum_{\substack{\alpha \in \mathbb{N}_{0}^{d},|\alpha|=\sigma}} \frac{\sigma !}{\alpha !} \int_{\mathbb{R}^{d}} \frac{1}{2} a(\rho)^{\sigma}\left(\rho\left|\partial^{\alpha} \hat{\mathbf{z}}_{1}\right|^{2}+\delta^{2} \rho\left|\partial^{\alpha} \hat{\mathbf{z}}_{\perp}\right|^{2}+g^{\prime}(\rho)\left(\partial^{\alpha} \hat{\rho}\right)^{2}\right) d z
$$


with $\rho=\varrho+\eta \hat{\rho}$. Under the assumptions of Proposition 3 and setting $\nabla^{\delta} \stackrel{\text { def }}{=}\left(\partial_{1}, \delta \nabla_{\perp}\right)$, we now have

$$
c\left(\|\hat{\mathbf{u}}\|_{H^{s}}^{2}+\|\hat{\rho}\|_{H^{s}}^{2}+\varepsilon^{2}\left\|\nabla^{\delta} \hat{\rho}\right\|_{H^{s}}^{2}\right) \leqslant E_{s}^{\natural}[\hat{\rho}, \hat{\mathbf{u}}] \leqslant C\left(\|\hat{\mathbf{u}}\|_{H^{s}}^{2}+\|\hat{\rho}\|_{H^{s}}^{2}+\varepsilon^{2}\left\|\nabla^{\delta} \hat{\rho}\right\|_{H^{s}}^{2}\right),
$$

where $c>0$ and $C>0$ depend only on $r, s, d$ (and the functions $g, K$ ).

The system $\left(\mathrm{ES}_{\varepsilon, \eta}\right)$ yields

$$
\partial_{T} \hat{\mathbf{z}}+\eta\left(\hat{\mathbf{u}} \cdot \nabla^{\delta}\right) \hat{\mathbf{z}}+i \eta\left(\nabla^{\delta} \hat{\mathbf{z}}\right) \hat{\mathbf{w}}+\frac{1}{\varepsilon} b(\rho) \hat{\mathbf{w}}+i \varepsilon \nabla^{\delta}\left(a(\rho) \nabla^{\delta} \cdot \hat{\mathbf{z}}\right)=0,
$$

and by applying the operator $\partial^{\alpha}$, where $|\alpha| \leqslant s$, we obtain

$$
\partial_{T} \partial^{\alpha} \hat{\mathbf{z}}+\eta\left(\hat{\mathbf{u}} \cdot \nabla^{\delta}\right) \partial^{\alpha} \hat{\mathbf{z}}+i \eta\left(\nabla^{\delta} \hat{\mathbf{z}}\right) \partial^{\alpha} \hat{\mathbf{w}}+\frac{1}{\varepsilon} b(\rho) \partial^{\alpha} \hat{\mathbf{w}}+i \varepsilon \partial^{\alpha} \nabla^{\delta}\left(a(\rho) \nabla^{\delta} \cdot \hat{\mathbf{z}}\right)=\mathbf{R},
$$

where

$$
\mathbf{R}=\left(\mathbf{R}_{1}, \delta \mathbf{R}_{\perp}\right) \stackrel{\text { def }}{=} \eta\left[\hat{\mathbf{u}} \cdot \nabla^{\delta}, \partial^{\alpha}\right] \hat{\mathbf{z}}+\frac{1}{\varepsilon}\left[b(\rho), \partial^{\alpha}\right] \hat{\mathbf{w}}+i \eta\left(\left(\nabla^{\delta} \partial^{\alpha} \hat{\mathbf{z}}\right) \hat{\mathbf{w}}-\partial^{\alpha}\left(\left(\nabla^{\delta} \hat{\mathbf{z}}\right) \hat{\mathbf{w}}\right)\right) .
$$

In view of (A.4), we have

$$
\left\|\left(\mathbf{R}_{1}, \delta \mathbf{R}_{\perp}\right)\right\|_{L^{2}} \leqslant C(r, s, d) \varepsilon^{2}\|(\nabla \hat{\mathbf{z}}, \nabla \hat{\rho})\|_{L^{\infty}} \sqrt{E_{s}^{\natural}[\hat{\rho}, \hat{\mathbf{z}}]},
$$

since, recalling that $\delta^{2} \leqslant \eta \leqslant 1$ and $\varepsilon^{2} \leqslant \eta \leqslant 1$ by (44),

$$
\begin{gathered}
\eta\left\|\left[\hat{\mathbf{u}} \cdot \nabla^{\delta}, \partial^{\alpha}\right] \hat{\mathbf{z}}\right\|_{L^{2}} \leqslant C(s, d) \eta\left(\|\nabla \hat{\mathbf{u}}\|_{H^{s-1}}\left\|\nabla^{\delta} \hat{\mathbf{z}}\right\|_{L^{\infty}}+\left\|\nabla^{\delta} \hat{\mathbf{z}}\right\|_{H^{s-1}}\|\nabla \hat{\mathbf{u}}\|_{L^{\infty}}\right) \\
\leqslant C(s, d) \eta\|\nabla \hat{\mathbf{z}}\|_{L^{\infty}} \sqrt{E_{s}^{\natural}[\hat{\rho}, \hat{\mathbf{z}}]}, \\
\eta\left\|\left(\nabla^{\delta} \partial^{\alpha} \hat{\mathbf{z}}\right) \hat{\mathbf{w}}-\partial^{\alpha}\left(\left(\nabla^{\delta} \hat{\mathbf{z}}\right) \hat{\mathbf{w}}\right)\right\|_{L^{2}} \leqslant C(s, d) \eta\left(\|\nabla \hat{\mathbf{w}}\|_{H^{s-1}}\left\|\nabla^{\delta} \hat{\mathbf{z}}\right\|_{L^{\infty}}+\left\|\nabla^{\delta} \hat{\mathbf{z}}\right\|_{H^{s-1}}\|\nabla \hat{\mathbf{w}}\|_{L^{\infty}}\right) \\
\leqslant C(s, d) \eta\|\nabla \hat{\mathbf{z}}\|_{L^{\infty}} \sqrt{E_{s}^{\natural}[\hat{\rho}, \hat{\mathbf{z}}]}
\end{gathered}
$$

and, using that $\|\hat{\mathbf{w}}\|_{H^{s-1}} \leqslant C(r, s, d) \varepsilon\|\hat{\rho}\|_{H^{s}} \leqslant C(r, s, d) \varepsilon \sqrt{E_{s}^{\natural}[\hat{\rho}, \hat{\mathbf{z}}]}$ and that $\|\hat{\mathbf{w}}\|_{L^{\infty}} \leqslant$ $C(r, s, d) \varepsilon\left\|\nabla^{\delta} \hat{\rho}\right\|_{L^{\infty}}$,

$$
\begin{aligned}
\left\|\frac{1}{\varepsilon}\left[b(\rho), \partial^{\alpha}\right] \hat{\mathbf{w}}\right\|_{L^{2}} & \leqslant C(r, s, d) \frac{\eta}{\varepsilon}\left(\|\hat{\mathbf{w}}\|_{H^{s-1}}\|\nabla \hat{\rho}\|_{L^{\infty}}+\|\hat{\mathbf{w}}\|_{L^{\infty}}\|\nabla \hat{\rho}\|_{H^{s-1}}\right) \\
& \leqslant C(r, s, d) \eta\|\nabla \hat{\rho}\|_{L^{\infty}} \sqrt{E_{s}^{\natural}[\hat{\rho}, \hat{\mathbf{z}}]} .
\end{aligned}
$$

Then, following the same lines as in the proof of Proposition 4 , but working with the variables (45) and the operator $\nabla^{\delta}=\left(\partial_{1}, \delta \nabla_{\perp}\right)$, we infer

$$
\frac{d}{d T} \dot{E}_{\sigma}^{\natural}[\hat{\rho}, \hat{\mathbf{z}}] \leqslant C(r, s, d) \eta\|(\nabla \hat{\rho}, \nabla \hat{\mathbf{z}})\|_{L^{\infty}}\left(1+\varepsilon \eta\|\nabla \hat{\rho}\|_{L^{\infty}}\right) E_{s}^{\natural}[\hat{\rho}, \hat{\mathbf{z}}] .
$$

This completes the proof of Theorem 8 . 


\subsection{Proof of Theorem 9 (ii)}

As already mentioned, the argument follows the lines of the proof of Theorem 1 in [21]. We briefly recall the ideas.

Construction of an approximate solution. We look for an approximate solution $\left(\hat{\rho}^{\mathrm{app}}, \hat{\mathbf{u}}^{\mathrm{app}}\right)$ to $\left(\mathrm{B}_{\varepsilon, \delta, \eta}\right)$ under the form

$$
\left(\hat{\rho}^{\mathrm{app}}, \hat{\mathbf{u}}^{\mathrm{app}}\right)(T, z)=\left(\hat{\rho}^{0}, \hat{\mathbf{u}}^{0}\right)(T, \eta T, z)+\eta\left(\hat{\rho}^{1}, \hat{\mathbf{u}}^{1}\right)(T, \eta T, z),
$$

where $\hat{\mathbf{u}}^{0}$ and $\hat{\mathbf{u}}^{1}$ are curl free. We set $\theta=\eta T$. Recall that $\delta^{2} \leqslant \eta$ and $\varepsilon^{2} \leqslant \eta$, and we wish to construct an approximate solution so that the consistency error is $o(\eta)$, since we consider $T \lesssim \eta^{-1}$. Notice that we simplify the computations by assuming an expansion in powers of $\varepsilon^{2}$, but an expansion in powers of $\varepsilon$ is also possible (see [21] in this case and also [11] if one considers only one wave propagating to the right). Then we compute

$$
\begin{aligned}
\operatorname{Err}_{\rho} \stackrel{\text { def }}{=} & \partial_{T} \hat{\rho}^{\text {app }}+\varrho \partial_{z_{1}} \hat{\mathbf{u}}_{1}^{\text {app }}+\eta \partial_{z_{1}}\left(\hat{\rho}^{\text {app }} \hat{\mathbf{u}}_{1}^{\text {app }}\right)+\delta^{2} \nabla_{z_{\perp}} \cdot\left(\left(\varrho+\eta \hat{\rho}^{\text {app }}\right) \hat{\mathbf{u}}_{\perp}^{\text {app }}\right) \\
= & \left(\partial_{T} \hat{\rho}^{0}+\varrho \partial_{z_{1}} \hat{\mathbf{u}}^{0}\right)+\eta\left(\partial_{T} \hat{\rho}^{1}+\varrho \partial_{z_{1}} \hat{\mathbf{u}}_{1}^{1}+\partial_{\theta} \hat{\rho}^{0}+\partial_{z_{1}}\left(\hat{\rho}^{0} \hat{\mathbf{u}}_{1}^{0}\right)+\varrho\left(\delta^{2} / \eta\right) \nabla_{z_{\perp}} \cdot \hat{\mathbf{u}}_{\perp}^{0}\right)+\mathcal{R}, \\
\operatorname{Err}_{1} \stackrel{\text { def }}{=} & \partial_{T} \hat{\mathbf{u}}_{1}^{\text {app }}+g^{\prime}(\varrho) \partial_{z_{1}} \hat{\rho}^{\text {app }}+\varepsilon^{2} \hat{\mathbf{u}}_{1}^{\text {app }} \partial_{z_{1}} \hat{\mathbf{u}}_{1}^{\text {app }}+\varepsilon^{4} \hat{\mathbf{u}}_{\perp}^{\mathrm{app}} \cdot \nabla_{z_{\perp}} \hat{\mathbf{u}}_{1}^{\text {app }}+\varepsilon^{2} g^{\prime \prime}(\varrho) \hat{\rho}^{\mathrm{app}} \partial_{z_{1}} \hat{\rho}^{\mathrm{app}} \\
& \quad-\varepsilon^{2} K(\varrho) \partial_{z_{1}}\left[\partial_{z_{1}}^{2}+\varepsilon^{2} \Delta_{z_{\perp}}\right] \hat{\rho}^{\mathrm{app}} \\
= & \left(\partial_{T} \hat{\mathbf{u}}_{1}^{0}+g^{\prime}(\varrho) \partial_{z_{1}} \hat{\mathbf{u}}_{1}^{0}\right)+\mathcal{S}_{1} \\
& \quad+\varepsilon^{2}\left(\partial_{T} \hat{\mathbf{u}}_{1}^{1}+g^{\prime}(\varrho) \partial_{z_{1}} \hat{\mathbf{u}}_{1}^{1}+\partial_{\theta} \hat{\mathbf{u}}_{1}^{0}+\hat{\mathbf{u}}_{1}^{0} \partial_{z_{1}} \hat{\mathbf{u}}_{1}^{0}+g^{\prime \prime}(\varrho) \partial_{z_{1}} \hat{\rho}^{0}-K(\varrho) \partial_{z_{1}}^{3} \hat{\rho}^{0}\right),
\end{aligned}
$$

and

$$
\begin{aligned}
\operatorname{Err}_{\perp} \stackrel{\text { def }}{=} & \partial_{T} \hat{\mathbf{u}}_{\perp}^{\mathrm{app}}+g^{\prime}(\varrho) \nabla_{z_{\perp}} \hat{\rho}^{\mathrm{app}}+\varepsilon^{2} \hat{\mathbf{u}}_{1}^{\mathrm{app}} \partial_{z_{1}} \hat{\mathbf{u}}_{\perp}^{\mathrm{app}}+\varepsilon^{4} \hat{\mathbf{u}}_{\perp}^{\mathrm{app}} \cdot \nabla_{z_{\perp}} \hat{\mathbf{u}}_{1}^{\mathrm{app}}+\varepsilon^{2} g^{\prime \prime}(\varrho) \hat{\rho}^{\mathrm{app}} \nabla_{z_{\perp}} \hat{\rho}^{\mathrm{app}} \\
& -\varepsilon^{2} K(\varrho) \partial_{z_{1}}\left[\partial_{z_{1}}^{2}+\varepsilon^{2} \Delta_{z_{\perp}}\right] \hat{\rho}^{\mathrm{app}} \\
= & \left(\partial_{T} \hat{\mathbf{u}}_{1}^{0}+g^{\prime}(\varrho) \partial_{z_{1}} \hat{\mathbf{u}}_{1}^{0}\right)+\mathcal{S}_{\perp} \\
& +\varepsilon^{2}\left(\partial_{T} \hat{\mathbf{u}}_{1}^{1}+g^{\prime}(\varrho) \partial_{z_{1}} \hat{\mathbf{u}}_{1}^{1}+\partial_{\theta} \hat{\mathbf{u}}_{1}^{0}+\hat{\mathbf{u}}_{1}^{0} \partial_{z_{1}} \hat{\mathbf{u}}_{1}^{0}+g^{\prime \prime}(\varrho) \partial_{z_{1}} \hat{\rho}^{0}-K(\varrho) \partial_{z_{1}}^{3} \hat{\rho}^{0}\right)
\end{aligned}
$$

where the error terms $\mathcal{R}$ and $\mathcal{S}$ will be explicited later on. The point is that we are not able to prove that $\hat{\mathbf{u}}_{\perp}^{1}$ remains of order one on the time intervals we consider.

Cancellation of the terms of formal order $\eta^{0}$ yields

$$
\partial_{T} \hat{\rho}^{0}+\varrho \partial_{z_{1}} \hat{\mathbf{u}}_{1}^{0}=\partial_{T} \hat{\mathbf{u}}_{1}^{0}+g^{\prime}(\varrho) \partial_{z_{1}} \hat{\rho}^{0}=0,
$$

with general solution $\left(\hat{\rho}^{0}, \varrho \mathfrak{c}^{-1} \hat{\mathbf{u}}_{1}^{0}\right)(T, \theta, z)=\mathcal{Z}^{+}\left(\theta, z_{1}-\mathfrak{c} T, z_{\perp}\right)(1,1)+\mathcal{Z}^{-}\left(\theta, z_{1}+\mathfrak{c} T, z_{\perp}\right)(1,-1)$ for some functions $\mathcal{Z}^{ \pm}$.

Cancellation of the terms of formal order $\eta$ provides

$$
\left\{\begin{array}{l}
\partial_{T} \hat{\rho}^{1}+\varrho \partial_{z_{1}} \hat{\mathbf{u}}_{1}^{1}+\partial_{z_{1}}\left(\hat{\rho}^{0} \hat{\mathbf{u}}_{1}^{0}\right)+\partial_{\theta} \hat{\rho}^{0}+\varrho\left(\delta^{2} / \eta\right) \nabla_{z_{\perp}} \cdot \hat{\mathbf{u}}_{\perp}^{0}=0 \\
\partial_{T} \hat{\mathbf{u}}_{1}^{1}+g^{\prime}(\varrho) \partial_{z_{1}} \hat{\rho}^{1}+\partial_{\theta} \hat{\mathbf{u}}_{1}^{0}+\hat{\mathbf{u}}_{1}^{0} \partial_{z_{1}} \hat{\mathbf{u}}_{1}^{0}+g^{\prime \prime}(\varrho) \partial_{z_{1}} \hat{\rho}^{0}-K(\varrho)\left(\varepsilon^{2} / \eta\right) \partial_{z_{1}}^{3} \hat{\rho}^{0}=0
\end{array}\right.
$$


Therefore, using the expressions for $\hat{\rho}^{0}$ and $\varrho \mathfrak{c}^{-1} \hat{\mathbf{u}}_{1}^{0}$,

$$
\begin{aligned}
\partial_{T} & \left(\hat{\rho}^{1}+\frac{\varrho}{\mathfrak{c}} \hat{\mathbf{u}}_{1}^{1}\right)+\mathfrak{c} \partial_{z_{1}}\left(\hat{\rho}^{1}+\frac{\varrho}{\mathfrak{c}} \hat{\mathbf{u}}_{1}^{1}\right) \\
= & -\partial_{\theta} \hat{\rho}^{0}-\frac{\varrho}{\mathfrak{c}} \partial_{\theta} \hat{\mathbf{u}}_{1}^{0}-\partial_{z_{1}}\left(\hat{\rho}^{0} \hat{\mathbf{u}}_{1}^{0}\right)-\varrho\left(\delta^{2} / \eta\right) \nabla_{z_{\perp}} \cdot \hat{\mathbf{u}}_{\perp}^{0}-\frac{\varrho}{\mathfrak{c}} \hat{\mathbf{u}}_{1}^{0} \partial_{z_{1}} \hat{\mathbf{u}}_{1}^{0} \\
& -\frac{\varrho}{\mathfrak{c}} g^{\prime \prime}(\varrho) \hat{\rho}^{0} \partial_{z_{1}} \hat{\rho}^{0}+\frac{\varrho}{\mathfrak{c}}\left(\varepsilon^{2} / \eta\right) K(\varrho) \partial_{z_{1}}^{3} \hat{\rho}^{0} \\
= & \left(-2 \partial_{\theta} \mathcal{Z}^{+}-2 \Gamma \mathcal{Z}^{+} \partial_{z_{1}} \mathcal{Z}^{+}-\mathfrak{c}\left(\delta^{2} / \eta\right) \Delta_{z_{\perp}} \partial_{z_{1}}^{-1} \mathcal{Z}^{+}+2 \kappa\left(\varepsilon^{2} / \eta\right) \partial_{z_{1}}^{3} \mathcal{Z}^{+}\right)\left(\theta, z_{1}-\mathfrak{c} T, z_{\perp}\right) \\
& +\left(-2(\Gamma-2 \mathfrak{c} / \varrho) \mathcal{Z}^{-} \partial_{z_{1}} \mathcal{Z}^{-}+\mathfrak{c}\left(\delta^{2} / \eta\right) \Delta_{z_{\perp}} \partial_{z_{1}}^{-1} \mathcal{Z}^{-}+2 \kappa\left(\varepsilon^{2} / \eta\right) \partial_{z_{1}}^{3} \mathcal{Z}^{-}\right)\left(\theta, z_{1}+\mathfrak{c} T, z_{\perp}\right) \\
& -\left(\frac{\mathfrak{c}}{\varrho}+2 \kappa\left(\varepsilon^{2} / \eta\right)\right) \partial_{z_{1}}\left[\mathcal{Z}^{+}\left(\theta, z_{1}-\mathfrak{c} T, z_{\perp}\right) \mathcal{Z}^{-}\left(\theta, z_{1}+\mathfrak{c} T, z_{\perp}\right)\right] .
\end{aligned}
$$

Then, $\hat{\rho}^{1}+\varrho c^{-1} \hat{\mathbf{u}}_{1}^{1}$ solves a transport equation with source terms. Notice that the first source term is a function of $z_{1}-\mathfrak{c} T$, thus is a solution to the associated homogeneous transport equation. Therefore, it has to vanish in order to remove secular growth (by the characteristic method). Hence,

$$
\partial_{\theta} \mathcal{Z}^{+}+\Gamma \mathcal{Z}^{+} \partial_{z_{1}} \mathcal{Z}^{+}+\frac{\mathfrak{c}}{2}\left(\delta^{2} / \eta\right) \Delta_{z_{\perp}} \partial_{z_{1}}^{-1} \mathcal{Z}^{+}-\kappa\left(\varepsilon^{2} / \eta\right) \partial_{z_{1}}^{3} \mathcal{Z}^{+}=0
$$

which is precisely the right-going KP-I equation: we then choose $\mathcal{Z}^{+}=\zeta^{+}$. In a symmetric way, we shall take $\mathcal{Z}^{-}=\zeta^{-}$. Recall that we assume $\Delta_{z_{\perp}} \zeta^{ \pm}(\theta=0) \in \partial_{z_{1}}^{2} H^{s+3}\left(\mathbb{R}^{d}\right)$. Therefore, $\partial_{\theta} \zeta^{ \pm} \in L^{\infty}\left(\left[0, \theta_{*}\right], \partial_{z_{1}} H^{s+3}\right)$ and $\Delta_{z_{\perp}} \zeta^{ \pm} \in L^{\infty}\left(\left[0, \theta_{*}\right], \partial_{z_{1}}^{2} H^{s+3}\right)$, as it follows from the arguments in [22] (see (3.9) and (3.10) there). Indeed, $\partial_{\theta} \zeta^{ \pm}$solves

$$
\partial_{\theta}\left(\partial_{\theta} \zeta^{ \pm}\right)+\Gamma \partial_{z_{1}}\left(\zeta^{ \pm}\left(\partial_{\theta} \zeta^{ \pm}\right)\right)+\frac{\mathfrak{c}}{2}\left(\delta^{2} / \eta\right) \Delta_{z_{\perp}} \partial_{z_{1}}^{-1}\left(\partial_{\theta} \zeta^{ \pm}\right)-\kappa\left(\varepsilon^{2} / \eta\right) \partial_{z_{1}}^{3}\left(\partial_{\theta} \zeta^{ \pm}\right)=0
$$

and $\partial_{\theta} \zeta^{ \pm}(\theta=0)=-\Gamma \partial_{z_{1}}\left(\left(\zeta^{ \pm}\right)^{2}(0) / 2\right)-\left(\mathfrak{c} \delta^{2} /(2 \eta)\right) \Delta_{z_{\perp}} \partial_{z_{1}}^{-1}\left(\zeta^{ \pm}(0)\right)+\left(\kappa \varepsilon^{2} / \eta\right) \partial_{z_{1}}^{3}\left(\zeta^{ \pm}(0)\right) \in$ $\partial_{z_{1}} H^{s+3}$ by assumption. Hence

$$
\begin{aligned}
\mathcal{F}\left(\partial_{\theta} \zeta^{ \pm}\right)(\theta)= & \exp \left(-i \theta\left(\kappa\left(\varepsilon^{2} / \eta\right) \xi_{1}^{3}+\mathfrak{c}\left(\delta^{2} / \eta\right)\left|\xi_{\perp}\right|^{2} /\left(2 \xi_{1}\right)\right)\right) \mathcal{F}\left(\partial_{\theta} \zeta^{ \pm}(0)\right) \\
& -i \Gamma \xi_{1} \int_{0}^{\theta} \exp \left(-i(\theta-\bar{\theta})\left(\kappa\left(\varepsilon^{2} / \eta\right) \xi_{1}^{3}+\mathfrak{c}\left(\delta^{2} / \eta\right)\left|\xi_{\perp}\right|^{2} /\left(2 \xi_{1}\right)\right)\right) \mathcal{F}\left(\zeta^{ \pm}\left(\partial_{\theta} \zeta^{ \pm}\right)\right)(\bar{\theta}) d \bar{\theta}
\end{aligned}
$$

where $\mathcal{F}$ denotes the Fourier transform. It then follows that $\partial_{\theta} \zeta^{ \pm} \in L^{\infty}\left(\left[0, \theta_{*}\right], \partial_{z_{1}} H^{s+3}\right)$ (and the argument does not depend on the space dimension). Consequently, we may rewrite the source term in the equation for $\hat{\rho}^{1}+\varrho \mathfrak{c}^{-1} \hat{\mathbf{u}}_{1}^{1}$ as a $z_{1}$-derivative:

$$
\begin{aligned}
\partial_{T}\left(\hat{\rho}^{1}+\frac{\varrho}{\mathfrak{c}} \hat{\mathbf{u}}_{1}^{1}\right)+\mathfrak{c} \partial_{z_{1}}\left(\hat{\rho}^{1}+\frac{\varrho}{\mathfrak{c}} \hat{\mathbf{u}}_{1}^{1}\right) \\
=\partial_{z_{1}}\left\{-(\Gamma-2 \mathfrak{c} / \varrho)\left[\zeta^{-}\right]^{2}\left(\theta, z_{1}+\mathfrak{c} T, z_{\perp}\right)+2 \kappa\left(\varepsilon^{2} / \eta\right) \partial_{z_{1}}^{2} \zeta^{-}\left(\theta, z_{1}+\mathfrak{c} T, z_{\perp}\right)\right. \\
\quad-\left(\frac{\mathfrak{c}}{\varrho}+2 \kappa\left(\varepsilon^{2} / \eta\right)\right) \zeta^{+}\left(\theta, z_{1}-\mathfrak{c} T, z_{\perp}\right) \zeta^{-}\left(\theta, z_{1}+\mathfrak{c} T, z_{\perp}\right) \\
\left.+\mathfrak{c}\left(\delta^{2} / \eta\right) \Delta_{z_{\perp}} \partial_{z_{1}}^{-2} \zeta^{-}\left(\theta, z_{1}+\mathfrak{c} T, z_{\perp}\right)\right\} .
\end{aligned}
$$


The characteristics method then yields

$$
\begin{aligned}
\left(\hat{\rho}^{1}+\frac{\varrho}{\mathfrak{c}} \hat{\mathbf{u}}_{1}^{1}\right)(T, \theta, z)= & \left(\hat{\rho}^{1, \text { in }}+\frac{\varrho}{\mathfrak{c}} \hat{\mathbf{u}}_{1}^{1, \text { in }}\right)\left(z_{1}-\mathfrak{c} T, z_{\perp}\right) \\
& +\frac{1}{2 \mathfrak{c}}(\Gamma-2 \mathfrak{c} / \varrho)\left[\zeta^{-}\right]^{2}\left(\theta, z_{1}-\mathfrak{c} T, z_{\perp}\right)-\frac{1}{2 \mathfrak{c}}(\Gamma-2 \mathfrak{c} / \varrho)\left[\zeta^{-}\right]^{2}\left(\theta, z_{1}+\mathfrak{c} T, z_{\perp}\right) \\
& +\frac{\kappa}{\mathfrak{c}}\left(\varepsilon^{2} / \eta\right) \partial_{z_{1}}^{2} \zeta^{-}\left(\theta, z_{1}+\mathfrak{c} T\right)-\frac{\kappa}{\mathfrak{c}}\left(\varepsilon^{2} / \eta\right) \partial_{z_{1}}^{2} \zeta^{-}\left(\theta, z_{1}-\mathfrak{c} T\right) \\
& -\left(\frac{1}{2 \varrho}+\frac{\kappa}{\mathfrak{c}}\left(\varepsilon^{2} / \eta\right)\right) \partial_{z_{1}}\left[\zeta^{+}\left(\theta, z_{1}-\mathfrak{c} T, z_{\perp}\right) \int_{z_{1}-\mathfrak{c} T}^{z_{1}+\mathfrak{c} T} \zeta^{-}\left(\theta, y, z_{\perp}\right) d y\right] \\
& +\frac{\delta^{2}}{2 \eta} \Delta_{z_{\perp}} \partial_{z_{1}}^{-2} \zeta^{-}\left(\theta, z_{1}+\mathfrak{c} T, z_{\perp}\right)-\frac{\delta^{2}}{2 \eta} \Delta_{z_{\perp}} \partial_{z_{1}}^{-2} \zeta^{-}\left(\theta, z_{1}-\mathfrak{c} T, z_{\perp}\right) .
\end{aligned}
$$

All the terms in the second, third and fifth lines in $(50)$ belong to $L^{\infty}\left(\left[0, \theta_{*}\right], H^{s+3}\right)$. For the term in the last line, we do not use (as in [21]) Proposition 3.6 in [20] for an estimate by $o(\sqrt{T})$, but write it under the form

$$
-\left(\frac{1}{2 \varrho}+\frac{\kappa}{\mathfrak{c}}\left(\varepsilon^{2} / \eta\right)\right) \partial_{z_{1}}\left[\zeta^{+}\left(\theta, z_{1}-\mathfrak{c} T, z_{\perp}\right)\left(\partial_{z_{1}}^{-1} \zeta^{-}\left(\theta, z_{1}+\mathfrak{c} T, z_{\perp}\right)-\partial_{z_{1}}^{-1} \zeta^{-}\left(\theta, z_{1}-\mathfrak{c} T, z_{\perp}\right)\right)\right],
$$

to see that is is bounded in $H^{s+3}$ by a constant uniformly for $0 \leqslant \theta, \eta T \leqslant \theta_{*}$. This allows us to derive the estimate

$$
\sup _{0 \leqslant T \leqslant \theta_{*} / \eta}\left\|\left(\hat{\rho}^{1}+\frac{\varrho}{\mathfrak{c}} \hat{\mathbf{u}}_{1}^{1}\right)(T, \eta T, \cdot)\right\|_{H^{s+3}} \leqslant C .
$$

In a similar way, we show that

$$
\sup _{0 \leqslant T \leqslant \theta_{*} / \eta}\left\|\left(\hat{\rho}^{1}-\frac{\varrho}{\mathfrak{c}} \hat{\mathbf{u}}_{1}^{1}\right)(T, \eta T, \cdot)\right\|_{H^{s+3}} \leqslant C,
$$

from which we deduce

$$
\sup _{0 \leqslant T \leqslant \theta_{*} / \eta}\left\|\left(\hat{\rho}^{1}, \hat{\mathbf{u}}_{1}^{1}\right)(T, \eta T, \cdot)\right\|_{H^{s+3}} \leqslant C .
$$

As a consequence, the approximate solution (49) enjoys the estimate

$$
\sup _{0 \leqslant T \leqslant \theta_{*} / \eta}\left\|\left(\hat{\rho}^{\mathrm{app}}, \hat{\mathbf{u}}^{\mathrm{app}}\right)(T)\right\|_{H^{s}} \leqslant C .
$$

The error terms $\mathcal{R}$ and $\mathcal{S}$ contain $\theta$-derivatives of $\hat{\rho}^{1}$ and $\hat{\mathbf{u}}_{1}^{1}$ that we wish to control. Let us observe that we have $\partial_{\theta} \zeta^{ \pm} \in L^{\infty}\left(\left[0, \theta_{*}\right], \partial_{z_{1}} H^{s+3}\right) \cap L^{\infty}\left(\left[0, \theta_{*}\right], H^{s+6}\right)$, but the direct differentiation of (50) with respect to $\theta$ would require to have $\partial_{\theta} \zeta^{ \pm} \in L^{\infty}\left(\left[0, \theta_{*}\right], \partial_{z_{1}}^{2} H^{s+2}\right)$, or at least $\Delta_{z_{\perp}} \partial_{\theta} \zeta^{ \pm} \in L^{\infty}\left(\left[0, \theta_{*}\right], \partial_{z_{1}}^{2} H^{s+2}\right)$. This is by no way possible if $d=2$ or 3 since the term $\Delta_{z_{\perp}} \partial_{z_{1}}^{-1}\left[\left(\zeta^{ \pm}\right)^{2}\right]$ appearing in $\partial_{\theta} \zeta^{ \pm}$is meaningless. Indeed, $\left(\zeta^{ \pm}\right)^{2} \in L^{1}$ has a Fourier 
transform which is continuous in $\mathbb{R}^{d}$ and positive at $\xi=0$ (unless $\zeta^{ \pm} \equiv 0$ ), but $\frac{\xi_{1}}{\left|\xi_{\perp}\right|^{2}}$ is not integrable near the origin for $d=2,3$. We thus proceed to the estimate for $\partial_{\theta}\left(\hat{\rho}^{1}, \hat{\mathbf{u}}_{1}^{1}\right)$ by first rewriting the term $\Delta_{z_{\perp}} \partial_{z_{1}}^{-2} \zeta^{-}\left(\theta, z_{1}+\mathfrak{c} T, z_{\perp}\right)-\Delta_{z_{\perp}} \partial_{z_{1}}^{-2} \zeta^{-}\left(\theta, z_{1}-\mathfrak{c} T, z_{\perp}\right)$ in the right-hand side of (50) under the form $\int_{z_{1}-\mathfrak{c} T}^{z_{1}+\mathfrak{c} T} \Delta_{z_{\perp}} \partial_{z_{1}}^{-1} \zeta^{-}\left(\theta, y, z_{\perp}\right) d y$. Consequently, by differentiation of 50 with respect to $\theta$, we obtain by using (51),

$$
\begin{aligned}
& \partial_{\theta}\left(\hat{\rho}^{1}+\frac{\varrho}{\mathfrak{c}} \hat{\mathbf{u}}_{1}^{1}\right) \\
= & \frac{1}{\mathfrak{c}}\left\{-(\Gamma-2 \mathfrak{c} / \varrho) \zeta^{+} \partial_{\theta} \zeta^{+}\left(\theta, z_{1}+\mathfrak{c} T, z_{\perp}\right)+(\Gamma-2 \mathfrak{c} / \varrho) \zeta^{-} \partial_{\theta} \zeta^{-}\left(\theta, z_{1}-\mathfrak{c} T, z_{\perp}\right)\right. \\
& \left.\quad+\kappa\left(\varepsilon^{2} / \eta\right) \partial_{z_{1}}^{2} \partial_{\theta} \zeta^{-}\left(\theta, z_{1}+\mathfrak{c} T, z_{\perp}\right)-\kappa\left(\varepsilon^{2} / \eta\right) \partial_{z_{1}}^{2} \partial_{\theta} \zeta^{-}\left(\theta, z_{1}-\mathfrak{c} T, z_{\perp}\right)\right\} \\
& -\left(\frac{1}{2 \varrho}+\frac{\kappa}{\mathfrak{c}}\left(\varepsilon^{2} / \eta\right)\right) \partial_{z_{1}} \partial_{\theta}\left[\zeta^{+}\left(\theta, z_{1}-\mathfrak{c} T, z_{\perp}\right)\left(\partial_{z_{1}}^{-1} \zeta^{-}\left(\theta, z_{1}+\mathfrak{c} T, z_{\perp}\right)-\partial_{z_{1}}^{-1} \zeta^{-}\left(\theta, z_{1}-\mathfrak{c} T, z_{\perp}\right)\right)\right] \\
& +\mathfrak{c}\left(\delta^{2} / \eta\right) \int_{-\mathfrak{c} T}^{+\mathfrak{c} T} \Delta_{z_{\perp}} \partial_{z_{1}}^{-1} \partial_{\theta} \zeta^{-}\left(\theta, y+z_{1}, z_{\perp}\right) d y,
\end{aligned}
$$

thus the estimates on $\zeta^{ \pm}$we have at hand and Proposition 3.6 in [20] for the last term yield

$$
\left\|\partial_{\theta}\left(\hat{\rho}^{1}+\frac{\varrho}{\mathfrak{c}} \hat{\mathbf{u}}_{1}^{1}\right)(T, \eta T, \cdot)\right\|_{H^{s}} \leqslant C+o(T)=o\left(\eta^{-1}\right) .
$$

Since a similar estimate holds true for $\partial_{\theta}\left(\hat{\rho}^{1}-\varrho \mathfrak{c}^{-1} \hat{\mathbf{u}}_{1}^{1}\right)$, we deduce

$$
\sup _{0 \leqslant T \leqslant \theta_{*} / \eta}\left\|\partial_{\theta}\left(\hat{\rho}^{1}, \hat{\mathbf{u}}_{1}^{1}\right)(T, \eta T, \cdot)\right\|_{H^{s}}=o\left(\eta^{-1}\right) .
$$

On the other hand, the formula 50 provides, since $\hat{\mathbf{u}}^{1}$ is curl free,

$$
\begin{aligned}
&\left(\nabla_{z_{\perp}} \hat{\rho}^{1}+\frac{\varrho}{\mathfrak{c}} \partial_{z_{1}} \hat{\mathbf{u}}_{\perp}^{1}\right)(T, \theta, z) \\
&=\left(\nabla_{z_{\perp}} \hat{\rho}^{1, \mathrm{in}}+\frac{\varrho}{\mathfrak{c}} \partial_{z_{1}} \hat{\mathbf{u}}_{\perp}^{1, \mathrm{in}}\right)\left(z_{1}-\mathfrak{c} T, z_{\perp}\right) \\
&+\frac{1}{2 \mathfrak{c}}\left\{-(\Gamma-2 \mathfrak{c} / \varrho) \partial_{z_{1}} \nabla_{z_{\perp}} \int_{z_{1}-\mathfrak{c} T}^{z_{1}+\mathfrak{c} T}\left[\zeta^{-}\right]^{2}\left(\theta, y, z_{\perp}\right) d y\right. \\
&+\mathfrak{c}\left(\delta^{2} / \eta\right) \partial_{z_{1}} \int_{z_{1}-\mathfrak{c} T}^{z_{1}+\mathfrak{c} T} \Delta_{z_{\perp}} \partial_{z_{1}}^{-2} \zeta^{-}\left(\theta, y, z_{\perp}\right) d y \\
&\left.\quad+2 \kappa\left(\varepsilon^{2} / \eta\right) \partial_{z_{1}}^{2} \nabla_{z_{\perp}}\left[\zeta^{-}\left(\theta, z_{1}+\mathfrak{c} T\right)-\zeta^{-}\left(\theta, z_{1}-\mathfrak{c} T\right)\right]\right\} \\
& \quad-\left(\frac{1}{2 \varrho}+\frac{\kappa}{\mathfrak{c}}\left(\varepsilon^{2} / \eta\right)\right) \partial_{z_{1}} \nabla_{z_{\perp}}\left[\zeta^{+}\left(\theta, z_{1}-\mathfrak{c} T, z_{\perp}\right) \int_{z_{1}-\mathfrak{c} T}^{z_{1}+\mathfrak{c} T} \zeta^{-}\left(\theta, y, z_{\perp}\right) d y\right]
\end{aligned}
$$

and a similar equality holds for $\nabla_{z_{\perp}} \hat{\rho}^{1}-\varrho \mathfrak{c}^{-1} \partial_{z_{1}} \hat{\mathbf{u}}_{\perp}^{1}$. Thus, taking the difference of the two equations and integrating in $z_{1}$, we obtain

$$
\sup _{0 \leqslant T \leqslant \theta_{*} / \eta}\left\|\hat{\mathbf{u}}_{\perp}^{1}(T, \eta T)\right\|_{H^{s}} \leqslant C+o(T)=o\left(\eta^{-1}\right),
$$


using once again Proposition 3.6 in [20] for the terms involving $\int_{z_{1}-\mathfrak{c} T}^{z_{1}+\mathfrak{c} T}$.

Let us now write more explicitly

$$
\begin{aligned}
\mathcal{R}= & \eta^{2} \partial_{\theta} \hat{\rho}^{1}+\eta^{2} \partial_{z_{1}}\left(\hat{\rho}^{1} \hat{\mathbf{u}}_{1}^{0}+\hat{\rho}^{0} \hat{\mathbf{u}}_{1}^{1}\right)+\delta^{2} \eta \nabla_{z_{\perp}} \cdot\left(\varrho \hat{\mathbf{u}}_{\perp}^{1}+\hat{\rho}^{0} \hat{\mathbf{u}}_{\perp}^{0}\right) \\
& +\eta^{2} \delta^{2} \nabla_{z_{\perp}} \cdot\left(\hat{\rho}^{0} \hat{\mathbf{u}}_{\perp}^{1}+\hat{\rho}^{1} \hat{\mathbf{u}}_{\perp}^{0}\right)+\eta^{3} \delta^{2} \nabla_{z_{\perp}} \cdot\left(\hat{\rho}^{1} \hat{\mathbf{u}}_{\perp}^{1}\right) .
\end{aligned}
$$

It follows from (52), (54) and (55) that

$$
\sup _{0 \leqslant T \leqslant T_{*} / \eta}\left\|\operatorname{Err}_{\rho}\right\|_{H^{s}} \leqslant \sup _{0 \leqslant T \leqslant \theta_{*} / \eta}\|\mathcal{R}\|_{H^{s}}=o(\eta) .
$$

Similarly, from the explicit relations

$$
\begin{aligned}
\mathcal{S}_{1}= & \eta^{2} \partial_{\theta} \hat{\mathbf{u}}_{1}^{1}+\eta^{2} \partial_{z_{1}}\left(\hat{\mathbf{u}}_{1}^{1} \hat{\mathbf{u}}_{1}^{0}\right)+\eta \delta^{2} \hat{\mathbf{u}}_{\perp}^{0} \cdot \nabla_{z_{\perp}} \hat{\mathbf{u}}_{1}^{0}+\eta^{2} g^{\prime \prime}(\varrho) \partial_{z_{1}}\left(\hat{\rho}^{1} \hat{\rho}^{0}\right)+\eta^{3} g^{\prime \prime}(\varrho) \hat{\rho}^{1} \partial_{z_{1}} \hat{\rho}^{1} \\
& -\varepsilon^{2} \eta K(\varrho) \partial_{z_{1}}^{3} \hat{\rho}^{1}-\varepsilon^{2} \delta^{2} K(\varrho) \partial_{z_{1}} \Delta_{z_{\perp}} \hat{\rho}^{0}-\eta^{2} \delta^{2} K(\varrho) \partial_{z_{1}} \Delta_{z_{\perp}} \hat{\rho}^{1} \\
& +\eta^{3} \hat{\mathbf{u}}_{1}^{1} \partial_{z_{1}} \hat{\mathbf{u}}_{1}^{1}+\eta^{2} \delta^{2} \hat{\mathbf{u}}_{\perp}^{1} \cdot \nabla_{z_{\perp}} \hat{\mathbf{u}}_{1}^{0}+\eta^{2} \delta^{2} \hat{\mathbf{u}}_{\perp}^{0} \cdot \nabla_{z_{\perp}} \hat{\mathbf{u}}_{1}^{1}+\eta^{3} \delta^{2} \hat{\mathbf{u}}_{\perp}^{1} \cdot \nabla_{z_{\perp}} \hat{\mathbf{u}}_{1}^{1},
\end{aligned}
$$

we infer

$$
\sup _{0 \leqslant T \leqslant T_{*} / \eta}\left\|\operatorname{Err}_{1}\right\|_{H^{s}}=o(\eta)
$$

and from

$$
\begin{aligned}
\mathcal{S}_{\perp}= & \eta^{2} \partial_{\theta} \hat{\mathbf{u}}_{\perp}^{1}+\eta^{2} \hat{\mathbf{u}}_{1}^{1} \partial_{z_{1}} \hat{\mathbf{u}}_{\perp}^{0}+\eta^{2} \hat{\mathbf{u}}_{1}^{0} \partial_{z_{1}} \hat{\mathbf{u}}_{\perp}^{1}+\eta \delta^{2} \hat{\mathbf{u}}_{\perp}^{0} \cdot \nabla_{z_{\perp}} \hat{\mathbf{u}}_{\perp}^{0}+\eta^{2} g^{\prime \prime}(\varrho) \nabla_{z_{\perp}}\left(\hat{\rho}^{1} \hat{\rho}^{0}\right) \\
& -\varepsilon^{2} \eta K(\varrho) \nabla_{z_{\perp}} \partial_{z_{1}}^{2} \hat{\rho}^{1}-\varepsilon^{2} \delta^{2} K(\varrho) \nabla_{z_{\perp}} \Delta_{z_{\perp}} \hat{\rho}^{0} \\
& +\eta^{3} \hat{\mathbf{u}}_{1}^{1} \partial_{z_{1}} \hat{\mathbf{u}}_{\perp}^{1}+\eta^{2} \delta^{2} \hat{\mathbf{u}}_{\perp}^{1} \cdot \nabla_{z_{\perp}} \hat{\mathbf{u}}_{\perp}^{0}+\eta^{2} \delta^{2} \hat{\mathbf{u}}_{\perp}^{0} \cdot \nabla_{z_{\perp}} \hat{\mathbf{u}}_{\perp}^{1}+\eta^{3} g^{\prime \prime}(\varrho) \hat{\rho}^{1} \nabla_{z_{\perp}} \hat{\rho}^{1}-\varepsilon^{2} \delta^{2} K(\varrho) \nabla_{z_{\perp}} \Delta_{z_{\perp}} \hat{\rho}^{1} \\
& +\eta^{3} \delta^{2} \hat{\mathbf{u}}_{\perp}^{1} \cdot \nabla_{z_{\perp}} \hat{\mathbf{u}}_{\perp}^{1},
\end{aligned}
$$

we deduce

$$
\sup _{0 \leqslant T \leqslant T_{*} / \varepsilon^{2}}\left\|\varepsilon \operatorname{Err}_{\perp}\right\|_{H^{s}}=o(\eta) .
$$

Comparison estimate. We shall follow the lines of the proof of Proposition 6. From (41) and the uniform bounds $(\hat{\rho}, \hat{\mathbf{u}}) \in \tilde{\mathcal{B}}_{s, \varepsilon, \delta}(2 M)$ for any $0 \leqslant T \leqslant T_{*} / \eta$, we infer that the difference $(\check{\rho}, \check{\mathbf{u}}) \stackrel{\text { def }}{=}(\hat{\rho}, \hat{\mathbf{u}})-\left(\hat{\rho}^{\text {app }}, \hat{\mathbf{u}}^{\text {app }}\right)$ satisfies the system

$$
\left\{\begin{array}{l}
\partial_{T} \check{\rho}+\nabla^{\delta} \cdot((\varrho+\eta \hat{\rho}) \check{\mathbf{u}})+\eta \nabla^{\delta} \cdot(\check{\rho} \hat{\mathbf{u}})=-\operatorname{Err}_{\tilde{\rho}} \\
\partial_{T} \check{\mathbf{u}}+g^{\prime}(\varrho) \nabla^{\delta} \check{\rho}+\eta \hat{\mathbf{u}} \cdot \nabla^{\delta} \check{\mathbf{u}}+\eta \check{\mathbf{u}} \cdot \nabla^{\delta} \tilde{\mathbf{u}}+\eta g^{\prime \prime}(\varrho) \hat{\rho} \nabla^{\delta} \check{\rho}+\eta g^{\prime \prime}(\varrho) \check{\rho} \nabla^{\delta} \tilde{\rho} \\
\quad=\varepsilon^{2} K(\varrho) \nabla^{\delta}\left[\partial_{z_{1}}^{2}+\delta^{2} \Delta_{z_{\perp}}\right] \check{\rho}-\operatorname{Err}_{\tilde{\mathbf{u}}}+\mathcal{O}_{L^{\infty}\left(\left[0, \min \left(T_{*}, \theta_{*}\right) / \eta\right], H^{s+3}\right)}\left(\eta\left(\eta+\varepsilon^{2}\right)\right) .
\end{array}\right.
$$

Similarly as in the proof of Proposition 6, we deduce that $0 \leqslant T \leqslant \min \left(T_{*}, \theta_{*}\right) / \eta$, there holds

$$
\begin{aligned}
\frac{d}{d T} E_{s-1}^{\sharp}(\check{\rho}, \check{\mathbf{z}}) \leqslant & C(s, r, d, M) \eta E_{s-1}^{\sharp}(\check{\rho}, \check{\mathbf{z}}) \\
& +C(s, r, d, M) \sqrt{E_{s-1}^{\sharp}}(\check{\rho}, \check{\mathbf{z}})\left(\left\|\left(\operatorname{Err}_{\tilde{\rho}}, \operatorname{Err}_{\tilde{\mathbf{u}}}, \varepsilon \nabla^{\delta} \operatorname{Err}_{\tilde{\rho}}\right)\right\|_{H^{s-1}}+\eta\left(\eta+\varepsilon^{2}\right)\right) .
\end{aligned}
$$


Gathering the consistency errors (56), (57), (58) we have established, we finally arrive by the Gronwall lemma at

$$
E_{s-1}^{\sharp}(\check{\rho}, \check{\mathbf{z}}) \leqslant C(s, r, d, M)\left(\eta+\varepsilon^{2}+o(1)\right),
$$

as wished.

\subsection{Proof of Theorem 10}

From (41) and the uniform bounds $(\hat{\rho}, \hat{\mathbf{u}}) \in \tilde{\mathcal{B}}_{s, \varepsilon, \delta}(2 M)$ for $0 \leqslant T \leqslant T_{*} / \eta$, we infer that

$$
\left\{\begin{aligned}
\partial_{T} \hat{\rho}+ & \nabla^{\delta} \cdot((\varrho+\eta \hat{\rho}) \hat{\mathbf{u}})=0 \\
\partial_{T} \hat{\mathbf{u}} & +\eta \hat{\mathbf{u}} \cdot \nabla^{\delta} \hat{\mathbf{u}}+g^{\prime}(\varrho) \nabla^{\delta} \hat{\rho}+\eta g^{\prime \prime}(\varrho) \hat{\rho} \nabla^{\delta} \hat{\rho}-\varepsilon^{2} K(\varrho) \nabla^{\delta}\left[\partial_{z_{1}}^{2}+\delta^{2} \Delta_{z_{\perp}}\right] \hat{\rho} \\
= & -\left(g^{\prime}(\varrho+\eta \hat{\rho})-g^{\prime}(\varrho)-\eta g^{\prime \prime}(\varrho) \hat{\rho}\right) \nabla^{\delta} \hat{\rho} \\
& +\varepsilon^{2} \nabla^{\delta}\left((K(\varrho+\eta \hat{\rho})-K(\varrho))\left[\partial_{z_{1}}^{2}+\delta^{2} \Delta_{z_{\perp}}\right] \hat{\rho}+\frac{\varepsilon^{2}}{2} K^{\prime}(\varrho+\eta \hat{\rho})\left|\nabla^{\delta} \hat{\rho}\right|^{2}\right) \\
= & \mathcal{O}_{L^{\infty}\left(\left[0, T_{*} / \eta\right], H^{s-3}\right)}\left(\eta\left(\eta+\varepsilon^{2}\right)\right) \quad \text { and } \quad \mathcal{O}_{L^{\infty}\left(\left[0, T_{*} / \eta\right], H^{s-2}\right)}(\eta(\eta+\varepsilon)) .
\end{aligned}\right.
$$

The conclusion then follows from the same arguments as those used for proving Theorem 9 . Notice that here, the error $\operatorname{Err}_{\rho}$ vanishes.

Acknowledgements: This work has been supported by the ANR project BoND (BondANR-13-BS01-0009-02).

\section{Appendix}

The estimates listed in the following proposition are rather standard for integer regularity index (see, e.g., [26], chapter 13, § 3) and for fractional regularity index, we can found: A.1) in [18] (Lemma X4 in the appendix there) or in [4] (Lemma B.1 in the appendix there); (A.3) in [4] (Lemma B.4) in the appendix there); (A.4) in [18] (Lemma X1 in the appendix there) or in [4] (Lemma A.2 in the appendix there).

Proposition A.1 • For $s \geqslant 0$, for all $u, v \in H^{s}\left(\mathbb{R}^{d}\right)$,

$$
\|u v\|_{H^{s}} \leqslant C(d, s)\left(\|u\|_{L^{\infty}}\|v\|_{H^{s}}+\|v\|_{L^{\infty}}\|u\|_{H^{s}}\right) .
$$

- For $s \in \mathbb{N}$, for all $u \in H^{s}\left(\mathbb{R}^{d}\right), v \in W^{s, \infty}\left(\mathbb{R}^{d}\right)$,

$$
\|u v\|_{H^{s}} \leqslant C(d, s)\|v\|_{W^{s, \infty}}\|u\|_{H^{s}} .
$$

- For $s>0$, if $F \in W^{\lceil s\rceil, \infty}([-r, r])$ vanishes at zero, for all $v \in H^{s}\left(\mathbb{R}^{d}\right)$ taking values in $[-r, r]$,

$$
\|F(v)\|_{H^{s}} \leqslant C(d, s, r)\left\|F^{\prime}\right\|_{W^{\lceil s\rceil-1, \infty}([-r, r])}\left(1+\|v\|_{L^{\infty}}\right)^{\lceil s\rceil}\|v\|_{H^{s}} .
$$

Here, $\lceil s\rceil$ is the smallest integer $m$ such that $m \geqslant s$. 
- For $s>0$, for all $u, v \in H^{s}\left(\mathbb{R}^{d}\right)$, for $\alpha \in \mathbb{N}^{d}$ such that $|\alpha| \leqslant s$,

$$
\left\|\partial^{\alpha}(u v)-u \partial^{\alpha} v\right\|_{L^{2}} \leqslant C(d, s)\left(\|\nabla u\|_{H^{s-1}}\|v\|_{L^{\infty}}+\|\nabla u\|_{L^{\infty}}\|v\|_{H^{s-1}}\right) .
$$

This is also true when $\partial^{\alpha}$ is replaced by $\Lambda^{s}=(1-\Delta)^{s / 2}$.

\section{References}

[1] W. Ben Youssef and T. Colin, Rigorous derivation of Korteweg-de Vries type systems from a general class of nonlinear hyperbolic systems. M2AN Math. Model. Numer. Anal. 34, no. 4 (2000), 873-911.

[2] W. Ben Youssef And D. LAnnes, The long wave limit for a general class of 2D quasilinear hyperbolic problems. Comm. Partial Differential Equations 27, no. 5-6 (2002), 979-1020.

[3] S. Benzoni-Gavage, Planar traveling waves in capillary fluids. Differential Integral Equations, 26, no. 3-4 (2013), 433-478.

[4] S. Benzoni-Gavage, R. Danchin and S. Descombes, On the well-posedness of the Euler-Korteweg model in several space dimensions. Indiana Univ. Math. J. 56 (2007), 1499-1579.

[5] S. Benzoni-Gavage, R. Danchin and S. Descombes, Well-posedness of onedimensional Korteweg models. Electron. J. Differential Equations, no. 59 (2006), 35 $p p$.

[6] F. Béthuel, R. Danchin and D. Smets, On the linear wave regime of the GrossPitaevskii equation. J. Anal. Math. 110 (2010), 297-338.

[7] F. Béthuel, P. Gravejat, J-C. Saut and D. Smets, On the Korteweg-de Vries long-wave transonic approximation of the Gross-Pitaevskii equation I. Internat. Math. Res. Notices, no. 14, (2009), 2700-2748.

[8] F. Béthuel, P. Gravejat, J-C. Saut and D. Smets, On the Korteweg-de Vries long-wave approximation of the Gross-Pitaevskii equation II. Comm. Partial Differential Equations 35, no. 1 (2010), 113-164.

[9] J.L. Bona, M. Chen And J.-C. Saut, Boussinesq equations and other systems for small-amplitude long waves in nonlinear dispersive media. Part I. Derivation and linear theory. J. Nonlinear Sci. 12 (2002), 283-318.

[10] J.L. Bona, T. Colin And D. LAnnes, Long wave approximations for water-waves. Arch. Ration. Mech. Anal. 178 (2005), 373-410. 
[11] D. Chiron, Error bounds for the (KdV)/(KP-I) and (gKdV)/(gKP-I) asymptotic regime for Nonlinear Schrödinger type Equations. Ann. Inst. H. Poincaré Anal. Non Linéaire 31, no. 6 (2014), 1175-1230.

[12] D. Chiron, Travelling waves for the Nonlinear Schrödinger Equation with general nonlinearity in dimension one. Nonlinearity 25 (2012), 813-850.

[13] D. Chiron, Three long-wave asymptotic regimes for the Nonlinear Schrödinger Equation. Singularities in Nonlinear Evolution Phenomena and Applications, M. Novaga 86 G. Orlandi Editors, CRM Series, Scuola Normale Superiore Pisa (2009), 107-138.

[14] D. Chiron and M. Mariş, Rarefaction pulses for the Nonlinear Schrödinger Equation in the transonic limit. Commun. Math. Phys. 326, no. 2 (2014), 329-392.

[15] D. Chiron and F. Rousset, The KdV/KP-I limit of the Nonlinear Schrödinger Equation. SIAM J. Math. Anal. 42, no. 1 (2010), 64-96.

[16] T. Colin And A. Soyeur, Some singular limits for evolutionary Ginzburg-Landau equations. Asymptotic Anal. 13 (1996), 361-372.

[17] P. Germain and F. Rousset, Long wave asymptotics for Schrödinger maps.

[18] T. Kato and G. Ponce, Commutator estimates and the Euler and Navier-Stokes equations. Comm. Pure Appl. Math. 41 (1988), no. 7, 891-90\%.

[19] D. Lannes, Consistency of the KP approximation. Dynamical systems and differential equations (Wilmington, NC, 2002). Discrete Contin. Dyn. Syst. (2003), 517-525 (suppl.).

[20] D. Lannes, Secular growth estimates for hyperbolic systems. J. Diff. Eqns. 190 (2003) 466-503.

[21] D. Lannes and J-C. Saut, Weakly transverse Boussinesq systems and the Kadomtsev-Petviashvili approximation. Nonlinearity 19 (2006), 2853-2875.

[22] L. Paumond, A rigorous link between KP and a Benney-Luke equation. Diff. Integral Eqns. 16 (2003), 1039-1064.

[23] J.-C. Saut, Remarks on the generalized Kadomtsev-Petviashvili equations. Indiana Univ. Math. J. 42 (1993), 1011-1026.

[24] J.-C. Saut and L. Xu, The Cauchy problem on large time for surface waves Boussinesq systems. J. Math. Pures Appl. 97 (2012), 635-662.

[25] G. Schneider and C.E. Wayne, The long-wave limit for the water wave problem. I. The case of zero surface tension. Comm. Pure Appl. Math. 53 (2000), no. 12, 1475-1535. Corrigendum: Comm. Pure Appl. Math. 65 (2012), no. 5, 587-591. 
[26] M. TAYlor, Partial Differential Equations. (III), Applied Mathematical Sciences, $11 \%$. Springer-Verlag, New-York, $199 \%$.

[27] S. UkaI, Local solutions of the Kadomtsev-Petviashvili equation. J. Fac. Sci. Univ. Tokyo IA (Math.) 36 (1989), 193-209. 